\title{
Unique Tube-Ring Interactions: Complexation of Single-Walled Carbon Nanotubes with Cycloparaphenyleneacetylenes
}

\section{AUTHOR(S):}

Miki, Koji; Saiki, Kenzo; Umeyama, Tomokazu; Baek, Jinseok; Noda, Takeru; Imahori, Hiroshi; Sato, Yuta; Suenaga, Kazu; Ohe, Kouichi

\section{CITATION:}

Miki, Koji ...[et al]. Unique Tube-Ring Interactions: Complexation of Single-Walled Carbon Nanotubes with Cycloparaphenyleneacetylenes. Small 2018, 14(26): 1800720.

\section{ISSUE DATE:}

2018-06-27

URL:

http://hdl.handle.net/2433/235352

\section{RIGHT:}

This is the peer reviewed version of the following article: K. Miki, K. Saiki, T. Umeyama, J. Baek, T. Noda, H. Imahori, Y. Sato, K. Suenaga, K. Ohe, "Unique Tube-Ring Interactions: Complexation of Single-Walled Carbon Nanotubes with Cycloparaphenyleneacetylenes", Small. 2018 Jun;14(26):e1800720, which has been published in final form at https://doi.org/10.1002/smll.201800720. This article may be used for non-commercial purposes in accordance with Wiley Terms and Conditions for Use of Self-Archived Versions.; The full-text file will be made open to the public on 27 June 2019 in accordance with publisher's 'Terms and Conditions for Self-Archiving'.; この論文は出版社版でありません 。引用の際には出版社版をご確認ご利用ください。; This is not the published version. Please cite only the published version. 


\section{WILEY-VCH}

DOI: 10.1002/ ((please add manuscript number))

Article type: Full Paper

\section{Unique Tube-Ring Interactions: Complexation of Single-Walled Carbon Nanotubes with Cycloparaphenyleneacetylenes}

Koji Miki, * Kenzo Saiki, Tomokazu Umeyama, Jinseok Baek, Takeru Noda, Hiroshi Imahori, Yuta Sato, Kazu Suenaga, and Kouichi Ohe*

Prof. K. Miki, K. Saiki, T. Noda, Prof. K. Ohe

Department of Energy and Hydrocarbon Chemistry, Graduate School of Engineering, Kyoto University, Katsura, Nishikyo-ku, Kyoto 615-8510, Japan

E-mail: kojimiki@scl.kyoto-u.ac.jp; ohe@scl.kyoto-u.ac.jp

Dr. J. Baek, Prof. T. Umeyama, Prof. H. Imahori

Department of Molecular Engineering, Graduate School of Engineering, Kyoto University, Katsura, Nishikyo-ku, Kyoto 615-8510, Japan

Prof. H. Imahori

Institute for Integrated Cell-Material Sciences (iCeMS), Kyoto University,

Yoshidaushinomiya-cho, Sakyo-ku, Kyoto 606-8501, Japan

Dr. Y. Sato, Dr. K. Suenaga

Nanomaterials Research Institute, National Institute of Advanced Industrial Science and Technology (AIST), Tsukuba, 305-8565, Japan

Keywords: carbon nanotube, carbon nanoring, supramolecular, rotaxane

Carbon nanotubes (CNTs) interlocked by cyclic compounds through supramolecular interaction are promising rotaxane-like materials applicable as two- and three-dimensional networks of nanowires and disease-specific theranostic agents having multi-functionalities. Supramolecular complexation of CNTs with cyclic compounds in a "ring toss" manner is a straightforward method to prepare interlocked CNTs; however, to date, this has not been reported on. Here, we report the first "ring toss" method to prepare interlocked CNTs by using $\pi$-conjugated carbon nanorings: [8]-, [9]-, and [10]cycloparaphenyleneacetylene (CPPA). CPPAs efficiently interact with CNTs to form CNT@CPPA complexes, while uncomplexed CPPAs can be recovered without decomposition. CNTs, which tightly fit in the cavities of CPPAs through convex-concave interaction, efficiently afford "tube-in-ring"-type CNT@CPPA complexes. “Tube-in-ring”-type and “ring-on-tube”-type complexation modes are successfully distinguished by spectroscopic, thermogravimetric, and microscopic analyses. 


\section{WILEY-VCH}

\section{Introduction}

Carbon nanotubes (CNTs) are considered to be one of the most important building blocks for the construction of nanometer-size intelligent electronic devices and smart biomaterials. ${ }^{[1]}$ To enhance not only their electrical and photophysical properties, but also their solubility, the covalent and noncovalent functionalization of CNTs has been intensively investigated. Much attention has been paid to the covalent functionalization, which lends a new function to CNTs; to some extent, it reduces the $\pi$-conjugation of pristine CNTs. ${ }^{[2]}$ However, only a limited number of noncovalent functionalization methods involving supramolecular interaction with CNTs, and which have no significant effect on the $\pi$-system, have been considered to date. Ever since the pioneering work describing a supramolecular method for dispersing fullerenes $\left(\mathrm{C}_{60}\right)$ inside CNTs was reported, ${ }^{[3]}$ related supramolecular functionalization inside CNTs has been intensively investigated. ${ }^{[4]}$ Functionalization of the outside of CNTs, achieved by supramolecular interaction of $\pi$-conjugated small molecules and polymers, has recently been investigated, although supramolecularly functionalized materials are inherently labile. ${ }^{[2 a, 5]}$ Interlocked supramolecular complexes of CNTs with cyclic compounds are considered to be applicable as two- and three-dimensional networks of nanowires and disease-specific theranostic agents having multiple functionalities.

Pérez and co-workers first reported the preparation of interlocked supramolecular complexes of CNT with thiofulvalene-containing macrocycles by the "nano-horseshoe throwing and clipping (ring-closing metathesis)" method (Figure 1a). ${ }^{[6]}$ The $\pi-\pi$ interaction between a CNT surface and electron-donating thiofulvalenes plays an important role in preparing such new mechanically interlocked CNT derivatives. This emerging method is an efficient one for preparing interlocked CNTs, but a significant loss of linear precursors and contamination with non-cyclized oligomers and polymers is unavoidable here. ${ }^{[7]}$ In an effort to overcome these problems, the "ring toss" method is considered as another approach to 


\section{WILEY-VCH}

preparing interlocked complexes of CNTs (Figure 1b). While a variety of rotaxanes are prepared by this method, from rod- and ring-shaped molecules, ${ }^{[8]}$ the "ring toss" method has, to date, not been applied to the preparation of interlocked complexes of CNTs, probably because of the weak interaction between non- $\pi$-conjugated macrocycles and CNTs.

a) Post-complexation macrocyclization [Pérez's work]

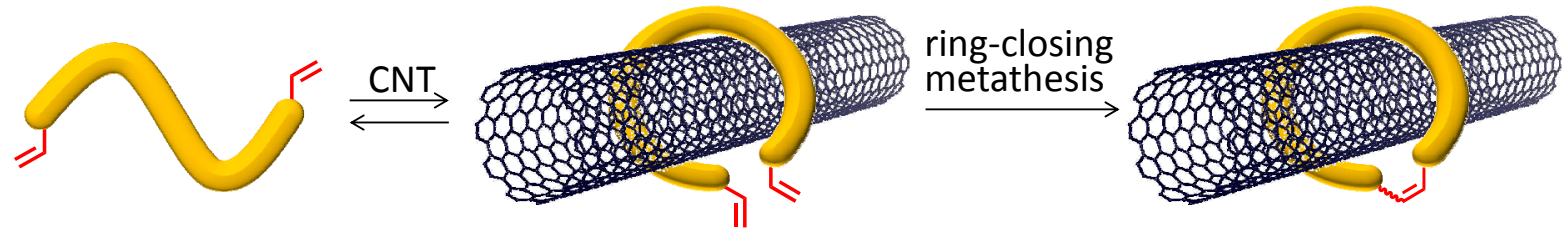

b) "Ring toss" method [this work]

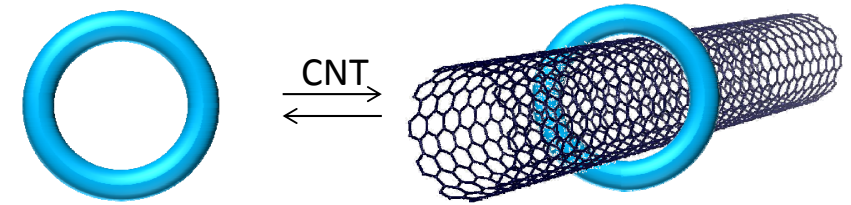

Figure 1. Formation of interlocked CNT complexes. a) Post-complexation macrocyclization method and b) "ring toss" method. Drawings of CNTs were produced by VESTA. ${ }^{[9]}$

Carbon nanorings, such as cycloparaphenyleneacetylene $(\mathrm{CPPA})^{[10]}$ and cycloparaphenylene $(\mathrm{CPP}),{ }^{[11]}$ are interesting $\pi$-conjugated macrocyclic compounds because of their specific photoluminescence properties and supramolecular inclusion properties (Figure $2 \mathrm{a}$ and $2 \mathrm{~b}$ ). It is reported that $\mathrm{C}_{60}$ can form supramolecular inclusion complexes with the carbon nanorings [6]CPPA ${ }^{[12]}$ and $[10] \mathrm{CPP},{ }^{[11 c]}$ respectively (Figure $2 \mathrm{c}$ and $2 \mathrm{~d}$ ). We have reported that the electron-rich [6]CPPA dyad containing anthracenes in the ring skeletons forms supramolecular inclusion complexes of two $\mathrm{C}_{60}$ molecules with high efficiency (Figure 2e). ${ }^{[13]}$ Interestingly, both $\mathrm{CPPAs}^{[14]}$ and $\mathrm{CPPs}^{[15]}$ are known to form inclusion complexes with their smaller-size carbon nanorings (Figure $2 \mathrm{f}$ and $2 \mathrm{~g}$ ). CPPs have twisted or helical structures because of the steric repulsion between the hydrogen atoms at 2,2'-positions of biphenyl moieties; ${ }^{[16]}$ therefore, almost all $[\mathrm{x}] \mathrm{CPP} @[\mathrm{x}+5] \mathrm{CPP}$ complexes $(\mathrm{x}=5,6,7,8$, and 10) adopt planetary orbital structures with incline angles of $18-55^{\circ}$. In contrast, Kawase and 


\section{WILEY-VCH}

co-workers have reported that "ring-in-ring"-type supramolecular complexes from [y]CPPAs and $[y+3]$ CPPAs $(y=5$ and 6$)$ are formed with high association constants, taking advantage of the tube-like structures of CPPAs with no steric repulsion.

a)

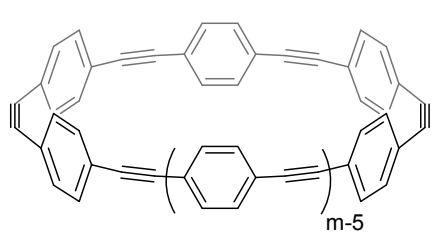

[m]cycloparaphenyleneacetylene ([m]CPPA)

c)

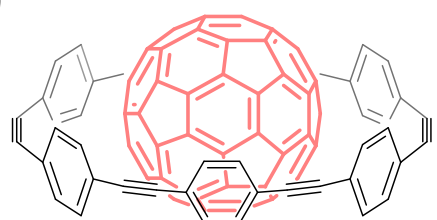

$\mathrm{C}_{60} @[6] \mathrm{CPPA}$

f)

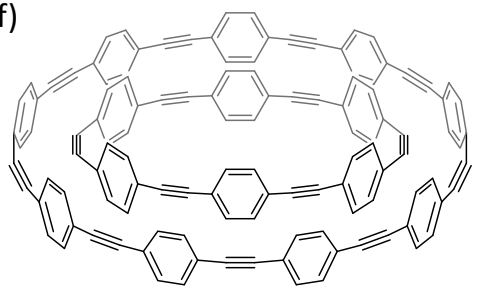

[6]CPPA@[9]CPPA

h)

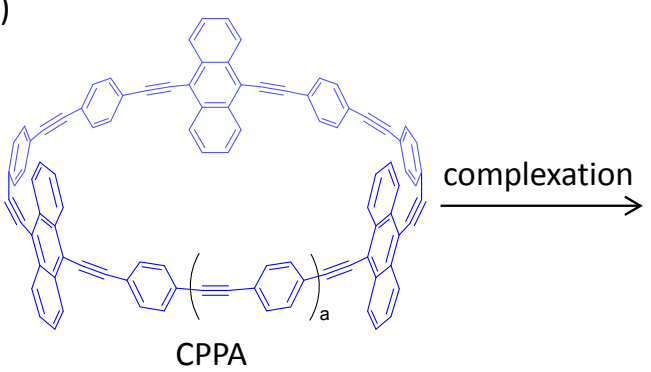

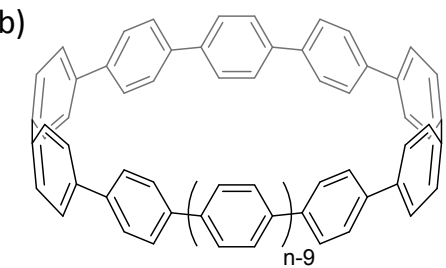

[n]cycloparaphenylene $([\mathrm{n}] \mathrm{CPP})$

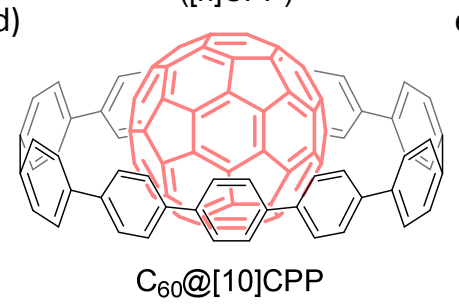

g)

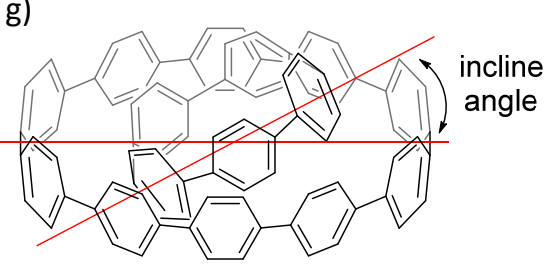

[5]CPP@[10]CPP

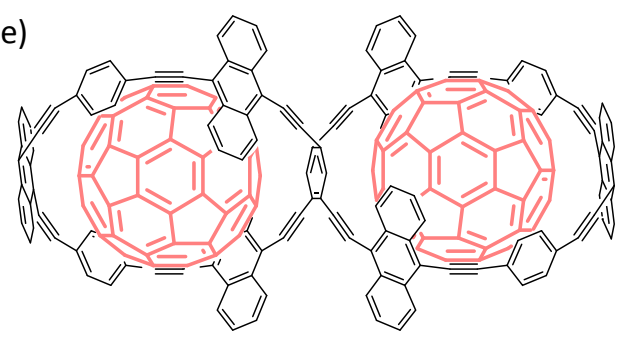

2C ${ }_{60} @[6] C P P A-d y a d$

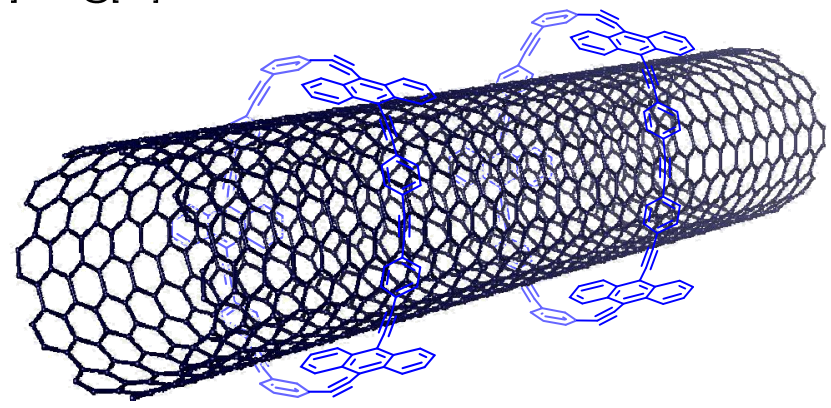

CNT@CPPA complexes

Figure 2. a) Cycloparaphenyleneacetylene (CPPA) and b) cycloparaphenylene (CPP). Complexes of $\mathrm{C}_{60}$ with c) [6]CPPA, d) [10]CPP, and e) [6]CPPA-dyad. "Ring-in-ring"-like complexes consisting of f) CPPAs and g) CPPs. h) CNT@CPPA complexes through "ring toss" method.

We propose a new avenue of access to interlocked CNT@CPPA complexes through the "ring toss" method, based on the strong inclusion ability of carbon nanorings, especially anthracene-containing CPPAs. Here, we describe the first example of the complexation of 


\section{WILEY-VCH}

CNTs with [8]-, [9]-, and [10]CPPAs 1 (Figure 2h). During the course of our study, we found that the complexation efficiency depends on the size of the CPPAs and the diameter of the CNTs. We were also able to distinguish between "tube-in-ring"-type and "ring-on-tube"-type complexes, based on high-resolution scanning probe microscopy (SPM) analysis.

\section{Results and Discussion}

\subsection{Synthesis of CPPAs 1 and their supramolecular complexation with CNTs}

[8]-, [9]-, and [10]CPPA derivatives 1a-d were successfully synthesized by a tin-mediated reductive aromatization reaction of their precursors, prepared via palladium-catalyzed Sonogashira-Hagihara coupling reactions in a stepwise manner (Figure 3a and see also the Supporting Information). CPPAs $\mathbf{1 b}-\mathbf{d}$ were isolated as orange solids and could be stored in a refrigerator in the dark for several months. A solution of CPPA 1a in a deoxygenated organic solvent could be stored in a refrigerator for a week; CPPA 1a in solid state gradually decomposed. From density functional theory (DFT) calculation at B3LYP/6-31G(d) level, ${ }^{[17]}$ the diameters of CPPAs 1a-d were estimated at $1.68 \mathrm{~nm}, 1.89 \mathrm{~nm}, 2.21 \mathrm{~nm}$, and $2.19 \mathrm{~nm}$, respectively (Figure 3b). 
a)

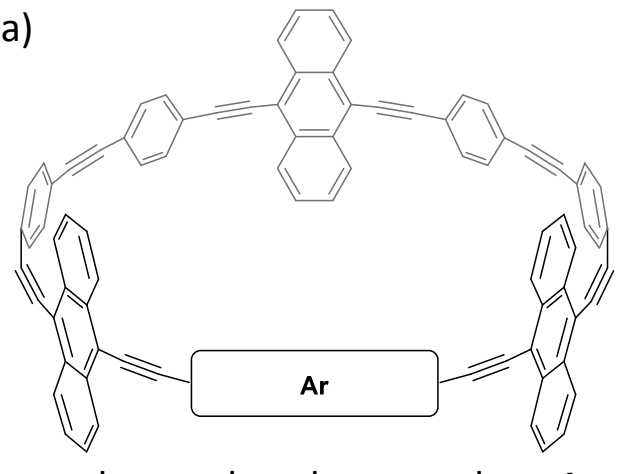
cycloparaphenyleneacetylene 1
[8]CPPA 1a $($ ar $=\longrightarrow)$

[9]CPPA 1b $($ ar $=\square=\square)$

[10]CPPA 1c $(\operatorname{Ar}=\cdots$

[10]CPPA 1d

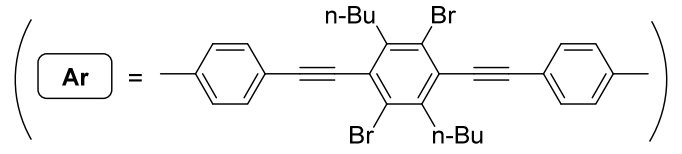

b)
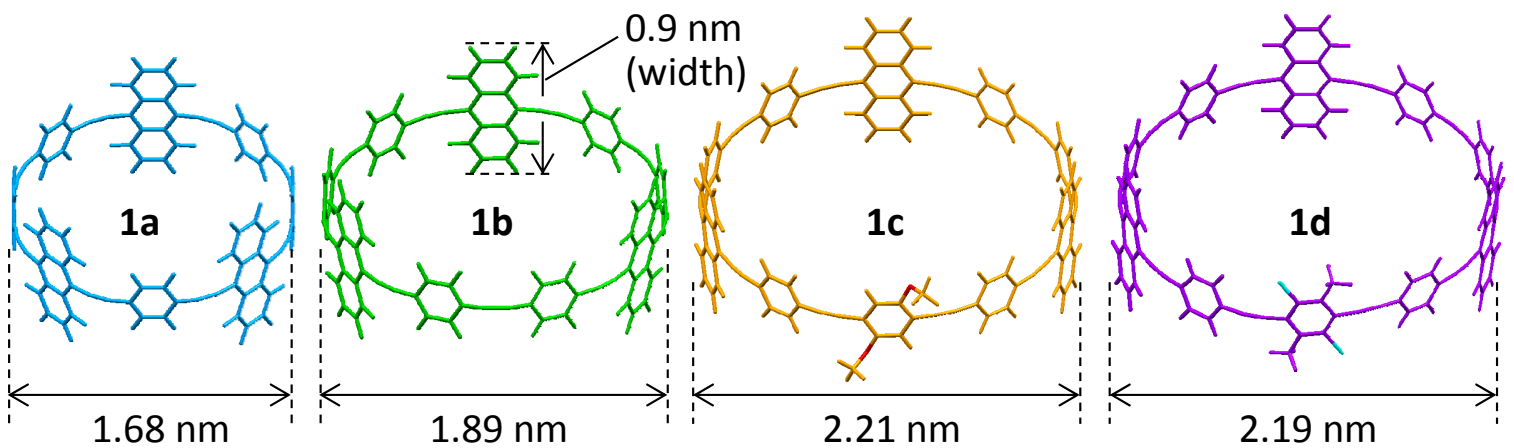

Figure 3. a) [8]-, [9]-, and [10]CPPA derivatives 1a-d. b) Structure of CPPAs 1a-d optimized at the B3LYP/6-31G(d) level of theory. Butoxy and butyl groups in 1c and 1d were replaced to methoxy and methyl groups, respectively, for reduction of calculation time.

The complexation of CPPAs $\mathbf{1}$ and single-walled carbon nanotubes CNTs A-C of various diameters was examined. Prior to using commercial CNTs, standard pretreatment ${ }^{[18]}$ was carried out to remove impurities. Data on the morphology and diameters of purified CNTs A-C were acquired by transmission electron microscope (TEM) and SPM. The diameters of CNTs A-C were about $0.7-1.0 \mathrm{~nm}, 1.1-1.4 \mathrm{~nm}$, and $1.2-1.6 \mathrm{~nm}$, respectively. The values did not change with pretreatment. Remnants were scarcely observed after pretreatment (Figure S19e,f and Figure S21 in the Supporting Information). Purified CNTs A-C were dispersed in $o$-dichlorobenzene (ODCB) by sonication and treated with 4 equivalents (by weight) of CPPAs for $18 \mathrm{~h}$ to prepare CNT@CPPA complexes 2 (Table 1). Regarding the numbering of the CNT@CPPA complexes, the number 2aA means that CNT@CPPA complex was prepared from CPPA 1a and CNT A. By measuring UV-vis absorbance of the recovered CPPAs, the amounts of complexed CPPAs were estimated. Untouched CPPAs were fully 


\section{WILEY-VCH}

recovered, unchanged. [8]- and [9]CPPA derivatives $\mathbf{1 a}(\phi=1.68 \mathrm{~nm})$ and $\mathbf{1 b}(\phi=1.89 \mathrm{~nm})$ are considered to form complexes with thin CNT A $(\phi=0.7-1.0 \mathrm{~nm})$ or CNT B $(\phi=1.1-1.4$ nm) (entries 1 and 7). This strongly supports that the good size fit is important for the formation of rotaxane-like structures of CNTs. The large CPPA 1c $(\phi=2.21 \mathrm{~nm})$ is considered to form a similar "tube-in-ring"-type complex with relatively thick CNT C $(\phi=$ 1.4-1.6 nm) or CNT B (entries 8 and 14). It is therefore reasonable that the combination of small CPPA 1a and thick CNT $\mathbf{B}$ or CNT $\mathbf{C}$ is unsuitable. Interestingly, the complexation of thin CNT A with large CPPA 1c did not proceed efficiently, although the diameter of 1c is large enough for complexation. No specific size dependence of $\mathbf{1 d}(\phi=2.19 \mathrm{~nm})$ was observed in the complexation ratio CNT ( 0.25 equiv):1d ( 1 equiv) (entries 4,9 , and 15). By increasing the amounts of CNTs used, it was found that the complexation between CNT B and $\mathbf{1 d}$ is more efficient than other cases (entries 5, 11, and 16). The use of five and ten times weighed CNT B for complexation with 1d increased the amounts of complexed CPPA 1d (entries 9-11). Taking the average diameters of the CNTs used into consideration (A: $0.8 \mathrm{~nm}$; B: $1.2 \mathrm{~nm} ; \mathbf{C}: 1.5 \mathrm{~nm}$ ), the average distance between two adjacent CPPAs associated with each CNT was calculated to be roughly $2-15 \mathrm{~nm}$ (Supporting Information, Table S7). 


\section{WILEY-VCH}

Table 1. Complexation of CPPA 1 with CNTs.

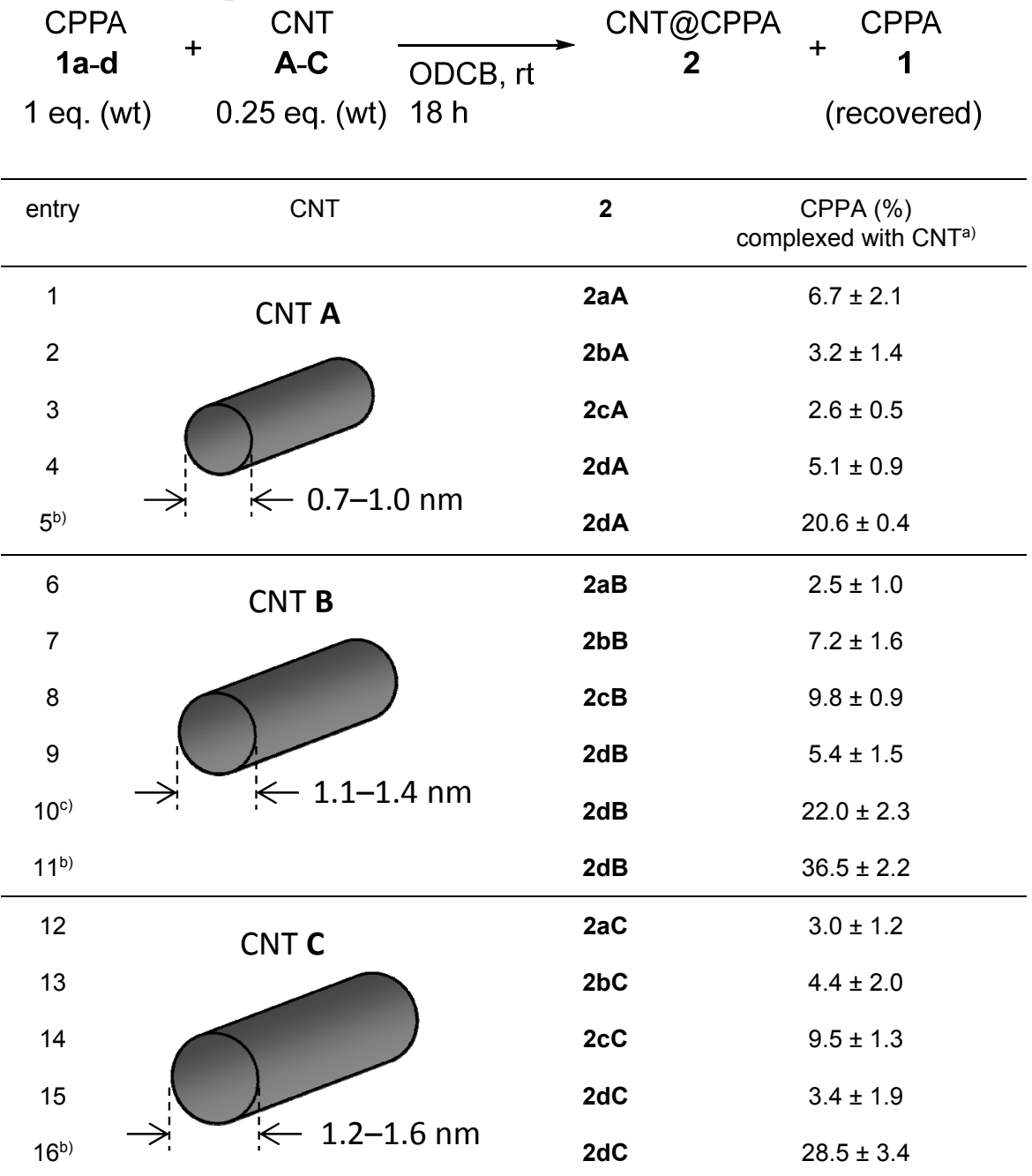

a) Calculated based on the amounts of CPPA recovered. Mean \pm s.d. $(\mathrm{n}=5) .{ }^{\text {b) }} \mathrm{CNT}$ (2.5 eq. (wt)) was used. ${ }^{\mathrm{c})}$ CNT B (1.25 eq. (wt)) was used.

This assessment and the size fit between CPPAs and CNTs indicates that there are two complexation modes in CNT@CPPA 2: "tube-in-ring” and "ring-on-tube". We also observed no dissociation of CPPAs from each CNT@CPPA 2, as confirmed by UV-vis absorption spectra, even after ODCB solutions of each CNT@CPPA were refluxed for several hours. This indicates that the noncovalent binding of CPPAs with CNTs is strong, irrespective of the complexation mode, "tube-in-ring" or "ring-on-tube". 


\subsection{CNT@CPPA complexes: Theoretical calculations}

\section{WILEY-VCH}

To predict the influence of CNT diameters on complexation with CPPAs 1a-d, the energyminimized molecular models of CNT@CPPA complexes were obtained by field force calculation with relatively inexpensive Forcite in Materials Studio software and MMFF94[19] developed by Merck, which are known to assess supramolecular interaction (including that of CNTs) with good structural accuracy. ${ }^{[6]}$ As shown in Figure 4, the diameters of the CNTs that can be interlocked by CPPAs $1 \mathbf{1 a - d}$ were estimated at $1.10 \mathrm{~nm}, 1.33 \mathrm{~nm}, 1.53 \mathrm{~nm}$, and 1.49 $\mathrm{nm}$, respectively. The representative "tube-in-ring" complex of $(14,0)-\mathrm{CNT}$ and 1a optimized with Forcite program is shown in Figure 5a. Interestingly, calculations showed that the ring sizes of CPPAs 1a and $\mathbf{1 b}$ could be expanded by $0.1 \mathrm{~nm}$ through complexation, whereas no expansion of 1c and 1d was found. Because of the steric hindrance of the bromine atoms as well as the butyl groups, the diameter of the CNT that gave the best fit with CPPA 1d is slightly smaller than that of 1c. The difference in size dependence between $1 \mathbf{c}$ and $\mathbf{1 d}$ in complexation is consistent with the quantification experiments (Table 1, vide supra). The "ring-on-tube" structure was also optimized by Forcite (Figure 5b). The "ring-on-tube" complexation is stabilized by $\mathrm{C}-\mathrm{H}-\pi$ interaction as well as $\pi-\pi$ interaction of one of three anthracene rings or one of three phenyl rings with a curved CNT surface. 


\section{WILEY-VCH}
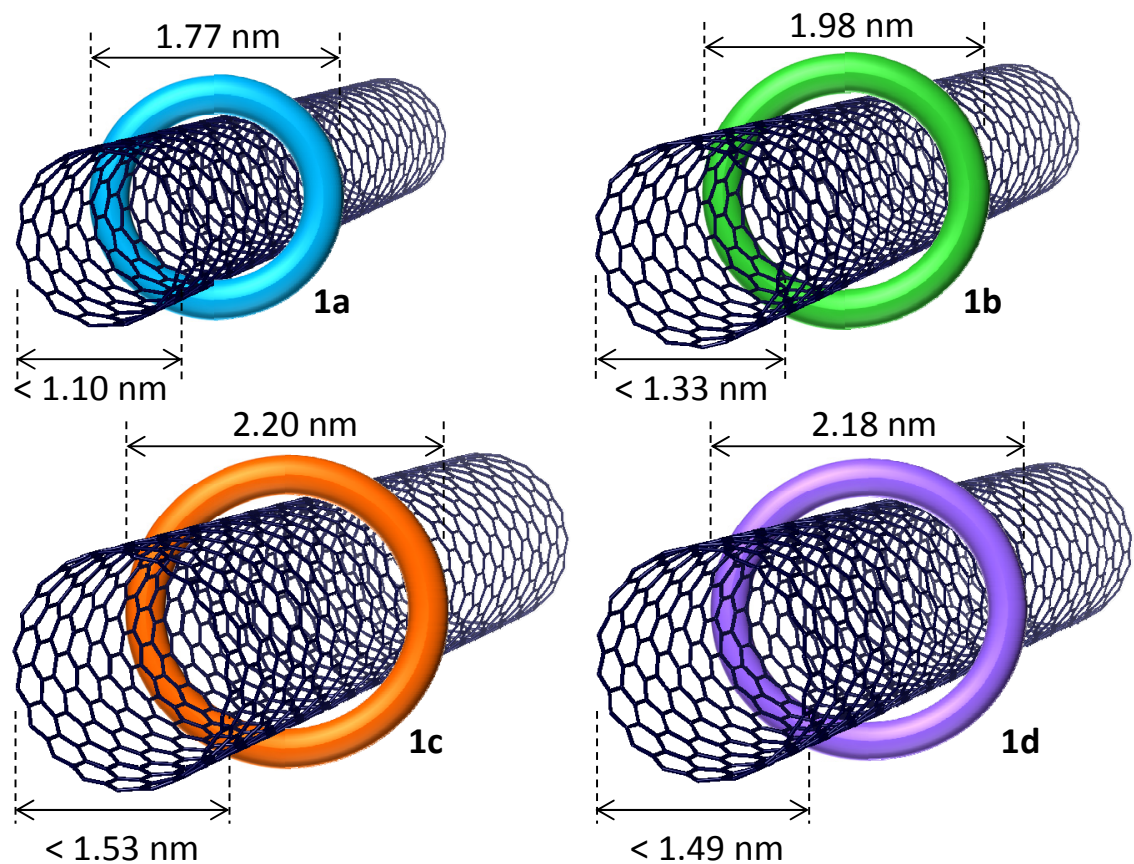

Figure 4. Schematic illustration of CNT@CPPA complexes. Diameters of CNTs which can be interlocked by CPPAs were calculated by Forcite and MMFF94.

a)

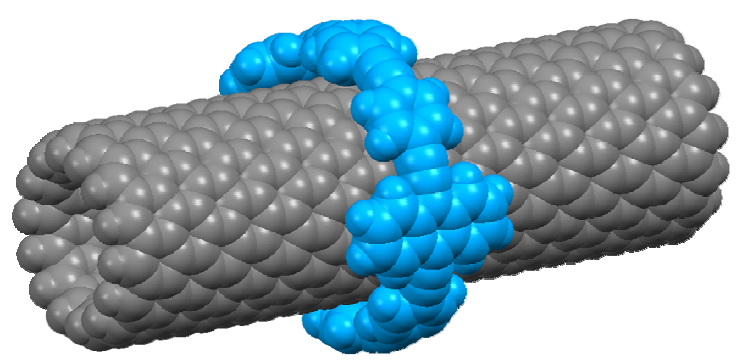

b)

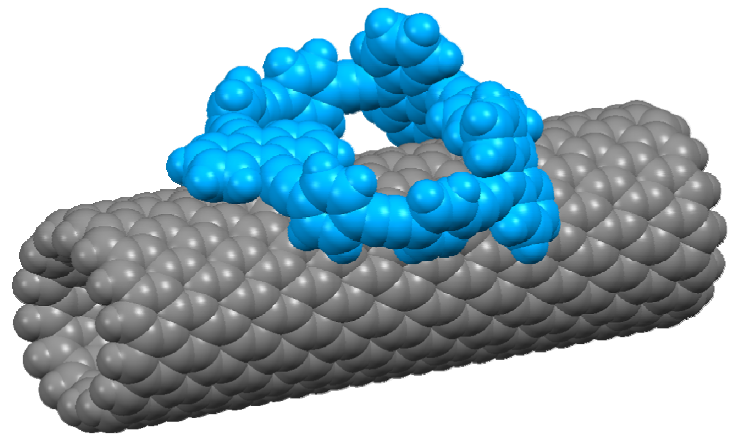

Figure 5. (a) "Tube-in-ring" and (b) "ring-on-tube" structures of CNT@CPPA complex of $(14,0) \mathrm{CNT}$ and 1a optimized with Forcite program and visualized by Mercury software (Mercury 3.6, provided by Cambridge Crystallographic Data Centre). Both terminals of CNT are capped with hydrogen atoms.

\section{3. "Tube-in-ring" supramolecular complexes: microscope observations}

We next gathered microscopic data on "tube-in-ring"-type complexes. High-resolution TEM revealed that, to our surprise, some of the CPPAs interacted with CNTs in the "tube-in-ring" manner (Figure 6a). The optimized structure of the CNT@CPPA complex of $(11,6)-\mathrm{CNT}$ and 1b molecules showed good agreement with the TEM image (Figure $6 \mathrm{~b}$ and $6 \mathrm{c}$ ). In an effort to define the complexation mode of CPPAs on CNTs, we attempted to identify the position of 


\section{WILEY-VCH}

the bromine atoms of $\mathbf{2} \mathbf{d C}$ in the TEM image; however, this was unsuccessful (the exact position of the bromine atoms could not be assigned).

a)

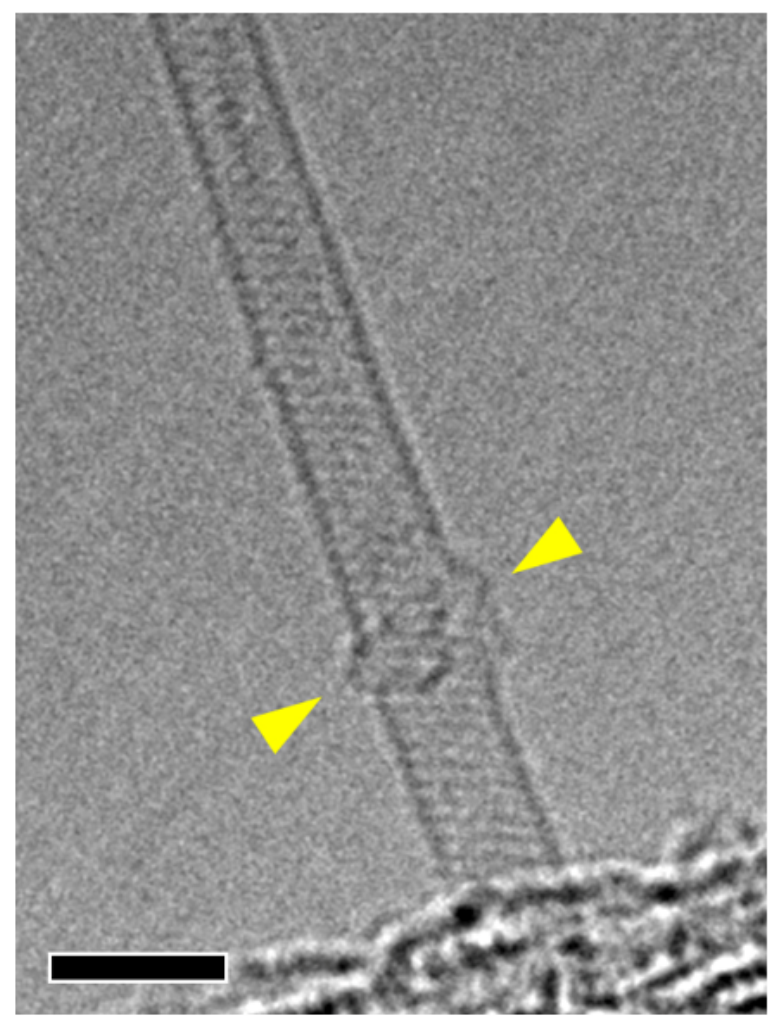

b)

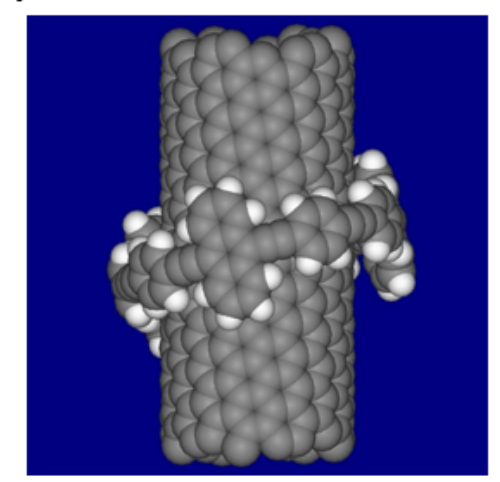

c)

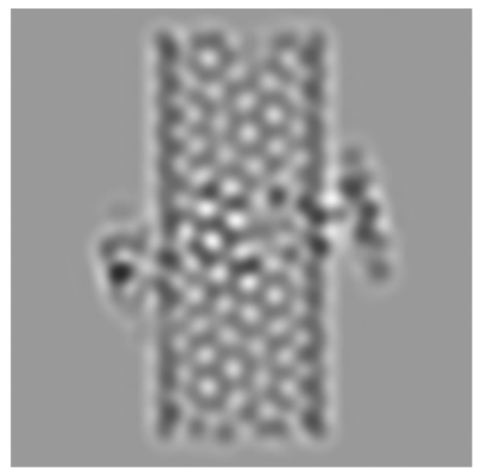

Figure 6. a) High-resolution TEM image of $\mathbf{2 b C}$. Scale bar, $2 \mathrm{~nm}$. b) Model structure of the complex of $(11,6)-\mathrm{CNT}$ and CPPA 1b. c) Corresponding TEM image simulation.

We then envisioned using SPM with sub-nanometer resolution on height to clarify the complexation modes. In the representative SPM image of $\mathbf{2 b A}$, some bright spots were seen on the CNTs (Figure 7a). The height profile analysis between two blue dots indicated that the height of the bright spot $\mathbf{P}$ was $1.89 \mathrm{~nm}$ (Figure $7 \mathrm{~b}$ ). Because the height of pristine CNT $\mathbf{A}$ was $1.06 \mathrm{~nm}$, according to the height profile analysis between two green dots (Figure 7c), the height difference $(0.83 \mathrm{~nm})$ between Figure $7 \mathrm{~b}$,c was considered to be provided by complexed CPPA. By taking the ring width $(0.9 \mathrm{~nm})$ of CPPA $\mathbf{1 b}$ into consideration, this bright spot could be assigned as a "ring-on-tube"-type mode (Figure 7i and Figure 5b). In other words, when the height difference $h_{1}$ between the CNT@CPPA complex and pristine CNT is $\sim 0.8$ $\mathrm{nm}$, the complexation mode could be assigned as a "ring-on-tube"-type (the height 


\section{WILEY-VCH}

distribution of $h_{1}$ is $\left.0.85 \pm 0.09 \mathrm{~nm}\right)$. In contrast, the height profile of the bright spot $\mathbf{Q}$ between two yellow dots showed a gentle slope from $0.72 \mathrm{~nm}$ (left) to $1.32 \mathrm{~nm}$ (right) (Figure 7d). This indicated that a CPPA ring is obliquely attached on the CNT surface (Figure $7 \mathrm{j}$ ). All the other bright spots observed in the measurement of $\mathbf{2} \mathbf{b A}$ exhibited these two patterns. In the case of $\mathbf{2 b B}$, several bright spots were also observed on CNTs (Figure 7e). From the height profile analyses between two blue dots and two green dots, the height gradually changed from $1.25 \mathrm{~nm}$ (CNT terminus) to $1.77 \mathrm{~nm}$ (bright spot $\mathbf{R}$ ) (Figure $7 \mathrm{f}$ and $7 \mathrm{~g}$ ). The height of the bump on the CNT surface was estimated at $0.38 \mathrm{~nm}$. Considering that the height difference $\left(h_{2}\right)$ of "tube-in-ring"-type complexation was less than $0.40 \mathrm{~nm}$, this profile suggests that the bright spot is assigned to CPPA complexed with CNT in the "tube-in-ring" manner (Figure 7k). A similar profile of the bright spots highlighted with red arrows was obtained and the height distribution of $h_{2}$ was $0.40 \pm 0.05 \mathrm{~nm}$ (Supporting Information, Figure S22a-e). Other SPM images of similar height profiles were also obtained together with other bright spots arising from CPPAs obliquely attached on the CNT surface (Supporting Information, Figure S22f-k). These results indicate that complex $\mathbf{2 b B}$ prepared from CPPA 1b $(\phi=1.89 \mathrm{~nm})$ and CNT B $(\phi=1.1-1.4 \mathrm{~nm})$ mainly contains the "tube-in-ring" mode in the complexation. 


\section{WILEY-VCH}

a)

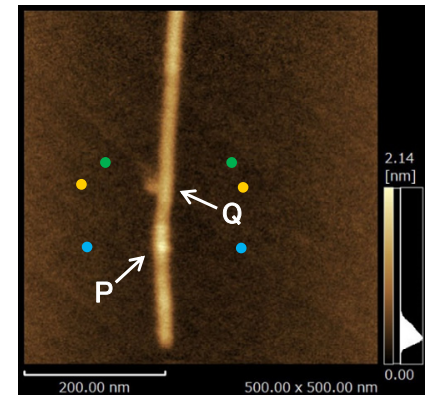

e)

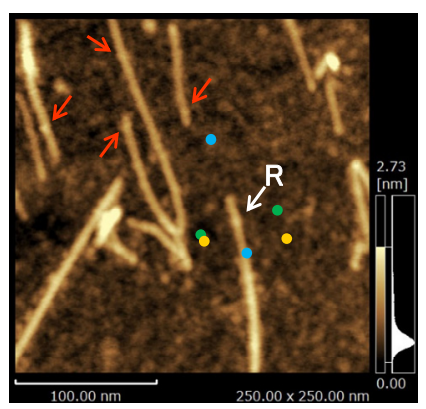

i)

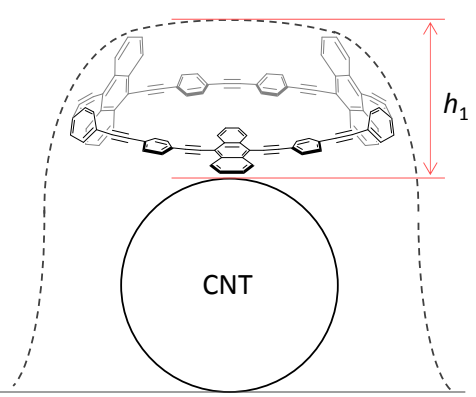

b)

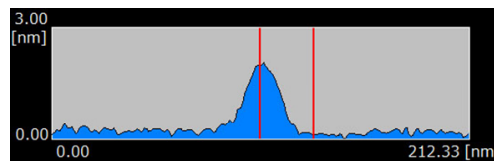

c)

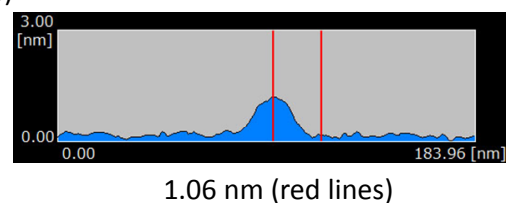

f)

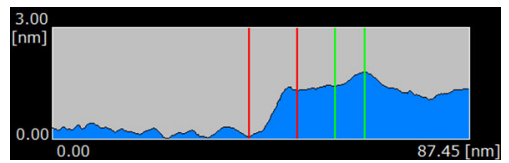

$1.25 \mathrm{~nm}$ (red lines), $0.38 \mathrm{~nm}$ (green lines) g)

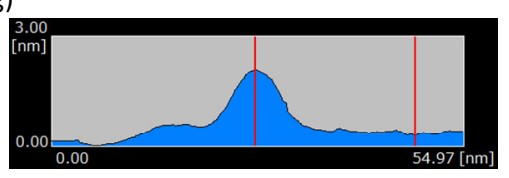

j)
$1.77 \mathrm{~nm}$ (red lines)

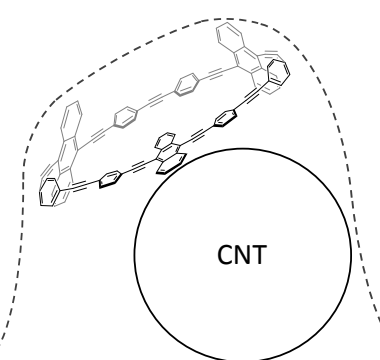

d)

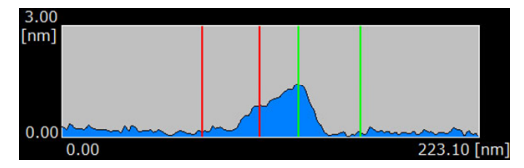

$0.72 \mathrm{~nm}$ (red lines), $1.32 \mathrm{~nm}$ (green lines)

h)

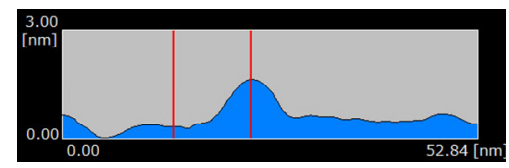

$1.30 \mathrm{~nm}$ (red lines)

k)

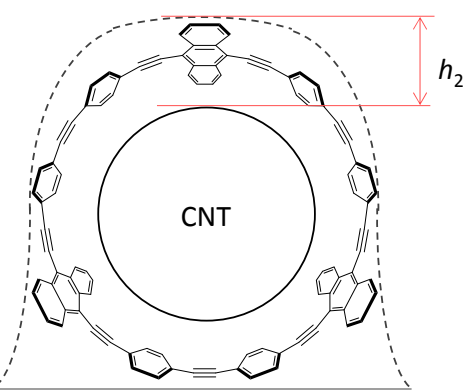

Figure 7. a) Representative SPM image of 2bA. Height profiles between two b) blue, c) green, and d) yellow dots. Height difference between two lines are shown. e) Representative SPM image of $\mathbf{2 b B}$. Height profiles between two f) blue, g) green, and h) yellow dots. Schematic illustration of estimated height profiles (dotted lines) of i) "Ring-on-tube"-type structure, j) a ring obliquely attached on a tube, and k) "tube-in-ring"-type structure.

\section{4. "Tube-in-ring" supramolecular complexation: plausible mechanism}

$\mathrm{C}_{60}$ is known to form "ball-on-bowl" and "ball-in-ring" inclusion complexes selectively with [6]CPPA (Figure 8a). ${ }^{[11,20]}$ Furthermore, [5]- and [6]CPPAs can be tightly accommodated in the cavity of [8]- and [9]CPPAs to construct the "ring-in-ring" complexes, respectively (Figure 2f). ${ }^{[14]}$ Taking these phenomena into consideration, we assumed that the "tube-inring" supramolecular complex CNT@CPPA may form in a "ring toss" manner upon the mixing of CPPAs and CNTs, regardless of capped-end and open-end structures of CNTs. ${ }^{[21]}$ We hypothesize that the CPPA rings slip into place outside the CNTs through an initial "ball- 


\section{WILEY-VCH}

on-bowl"-like and/or "ring-in-ring"-type interaction between the CPPA and CNT terminus, to ring the CNT (Figure 8b).

a)

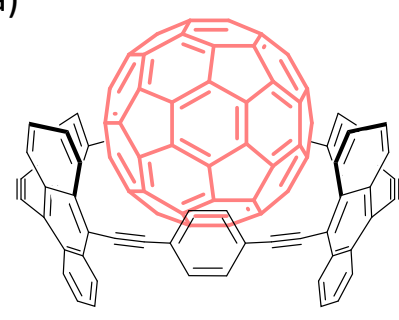

"ball-on-bowl"

b)

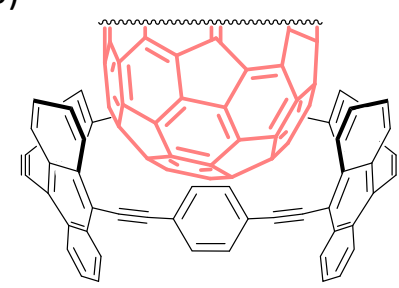

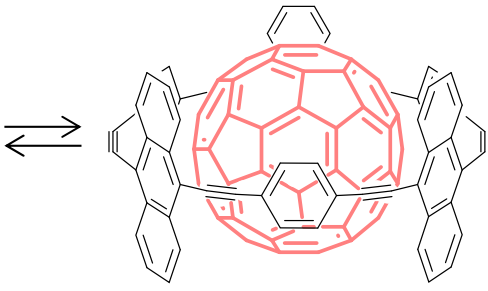

"ball-in-ring"

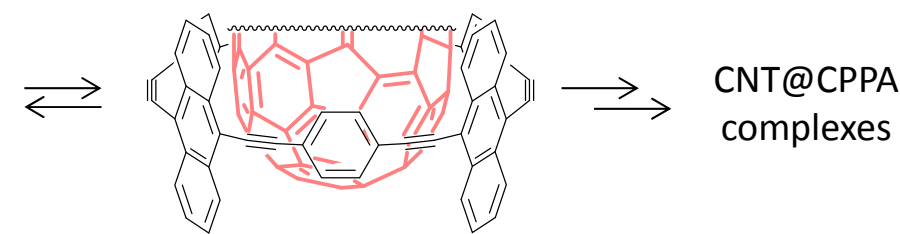

Figure 8. Complexation mechanism. a) Dynamic behavior of $\mathrm{C}_{60} @$ CPPA complexes. b) Plausible mechanism to form CNT@CPPA complexes.

\section{5. "Tube-in-ring" supramolecular complexes: thermogravimetric and spectroscopic analyses}

To distinguish the complexation modes, thermogravimetric analysis (TGA) of CNT@CPPA complexes $\mathbf{2}$ was applied. In the case of $\mathbf{2} \mathbf{b A}$, the weight gradually decreased during heating from $150{ }^{\circ} \mathrm{C}$ to $350{ }^{\circ} \mathrm{C}$ (Figure 9a). In the case of $\mathbf{2 b C}$, a similar weight loss of $15 \%$ at 150 $350{ }^{\circ} \mathrm{C}$ was observed, followed by a second gradual weight loss at $400{ }^{\circ} \mathrm{C}$ (Figure 9c). In contrast to these two complexes, in the case of $\mathbf{2 b B}$, only $20 \%$ weight loss at $400{ }^{\circ} \mathrm{C}$ was clearly detected by TGA and no weight loss at $150-350{ }^{\circ} \mathrm{C}$ was observed (Figure $9 \mathrm{~b}$ ). The amounts of weight loss observed at $400{ }^{\circ} \mathrm{C}$ were consistent with the CPPA contents of $2 \mathrm{aB}$ (9 $w t \%), \mathbf{2 b B}(22 \mathrm{wt} \%)$, and $\mathbf{2 c B}(28 \mathrm{wt} \%)$, estimated in quantification analyses (Table 1, entries 6-8). It was therefore concluded that both the first and second weight losses are caused by CPPA dissociation from CNTs. Because CPPA 1b gradually decomposed at $200{ }^{\circ} \mathrm{C}$, the 


\section{WILEY-VCH}

weight loss observed at $150-350^{\circ} \mathrm{C}$ could be assigned to the loss of CPPA $\mathbf{1 b}$ attached on the CNT surface in the "ring-on-tube" manner, while the degradation of CPPAs in the "tube-inring" manner would cause the second weight loss. TGA also revealed this two-step weight loss for other CNT@CPPA complexes 2 (Supporting Information, Figure S15). Taking the TGA results into consideration, we conclude that CNT@CPPA complex 2bB hardly contains CPPAs attached on the CNT surface in the "ring-on-tube" manner.

a)

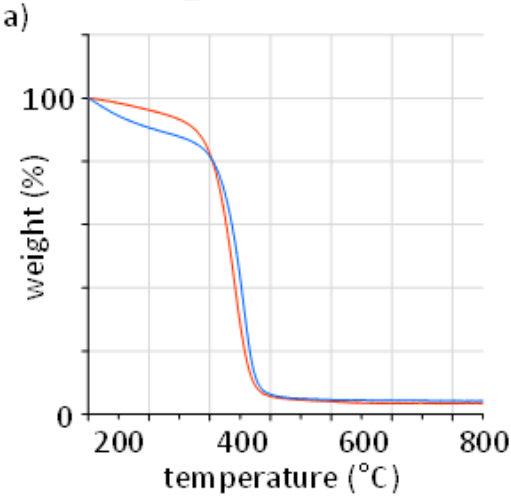

d)

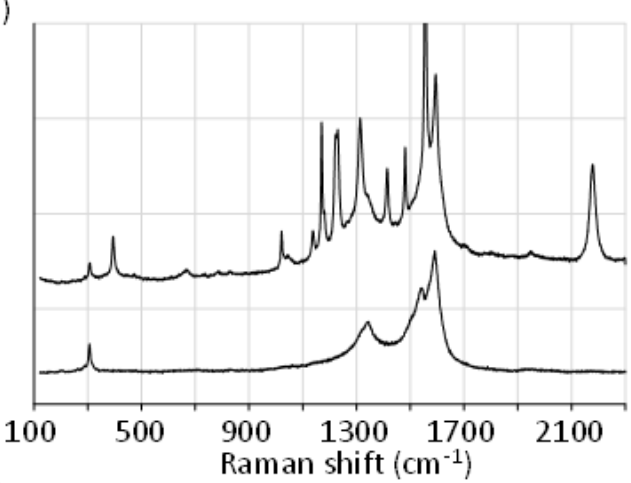

f)

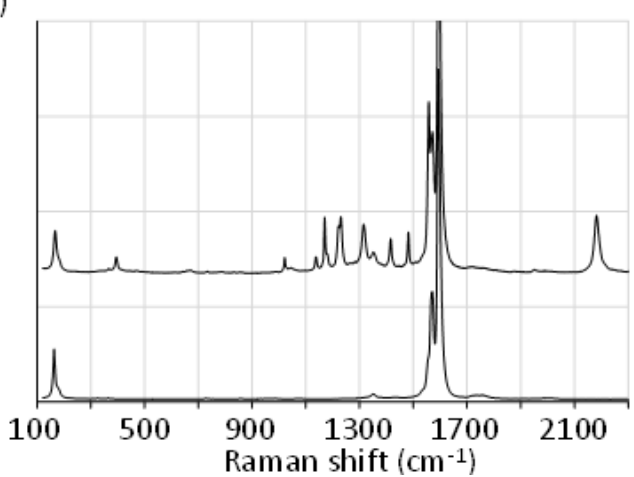

b)

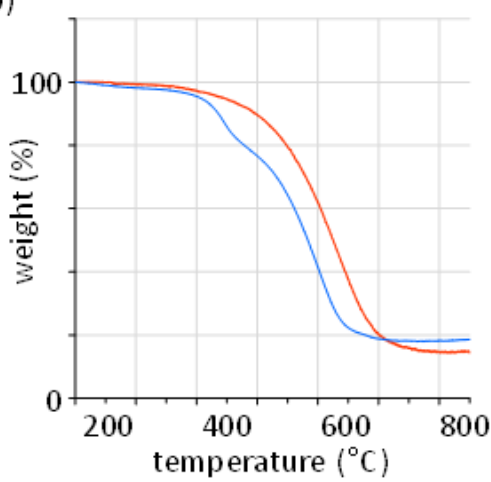

c)

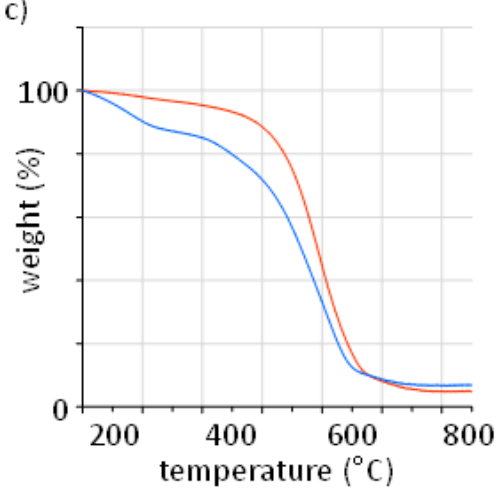

e)

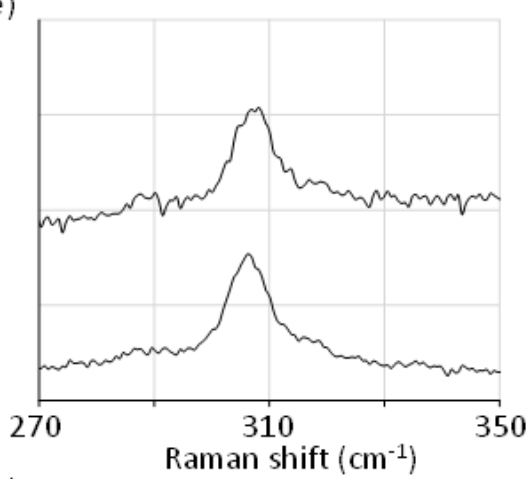

g)

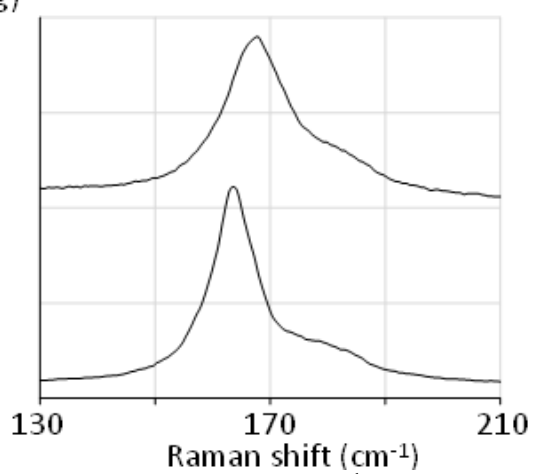

Figure 9. TGA of CNT@CPPAs. TGA (Flow: air $\left(50 \mathrm{~mL} \mathrm{~min}^{-1}\right)$, scan rate: $\left.2{ }^{\circ} \mathrm{C} \mathrm{min}^{-1}\right)$ of a) $\mathbf{2 b A}$, b) 2bB, and c) $\mathbf{2 b C}$ prepared through complexation for $24 \mathrm{~h}$. Purified pristine CNTs (red) and CNT@CPPA complexes 2 (blue). Raman spectra of CNT@CPPAs (excitation wavelength: $488 \mathrm{~nm}$ ). d) CNT A (bottom) and 2bA (up) and e) the enlarged Raman spectra in the region of RBM, and f) CNT C (bottom) and $\mathbf{2 b C}$ (up) and g) the enlarged Raman spectra in the region of RBM. 


\section{WILEY-VCH}

We next measured Raman spectra of 2 . It has been reported that the signal of the radial breathing mode (RBM) of CNTs wrapped with $\pi$-conjugated molecules and polymers shifts upfield to higher wavenumbers. ${ }^{[51]}$ In Raman spectra of CNT A and CNT@CPPA complex $\mathbf{2 b A}$, the RBM signal at $307 \mathrm{~cm}^{-1}$ was not shifted before/after complexation of CNT A with 1b (Figure 9d and 9e). RBM peak shifts of CNTs from $163 \mathrm{~cm}^{-1}$ to $167 \mathrm{~cm}^{-1}$ and from 192 $\mathrm{cm}^{-1}$ to $195 \mathrm{~cm}^{-1}$ were observed in the spectra of $\mathbf{2 b B}$ and $\mathbf{2 b C}$, respectively (Figure $9 \mathrm{f}$ and $9 \mathrm{~g}$, and Figure S17 in the Supporting Information). These data are consistent with the results of TGA, suggesting that CNT@CPPA complexes $2 \mathbf{b B}$ and $\mathbf{2 b C}$ contain “tube-in-ring” structures. RBM upfield shifts of $\mathbf{2 a A}, \mathbf{2 c C}, \mathbf{2 d B}$, and $\mathbf{2 d C}$ were also observed (Supporting Information, Figure S16, S18, and S19). These shifts in the Raman spectra of CNT@CPPA complexes suggest that CPPAs tightly interact with CNT through relatively strong convex-concave interaction.

Finally, complexation modes in CNT@CPPA complexes 2 are summarized in Table 2. Indications are that CPPAs having both larger and smaller ring sizes mainly form "ring-ontube"-type complexes with CNTs, whereas CPPAs that have a close fit with CNTs efficiently afford "tube-in-ring"-type complexes. 


\section{WILEY-VCH}

Table 2. Complexation Modes in CNT@CPPA Complexes 2.

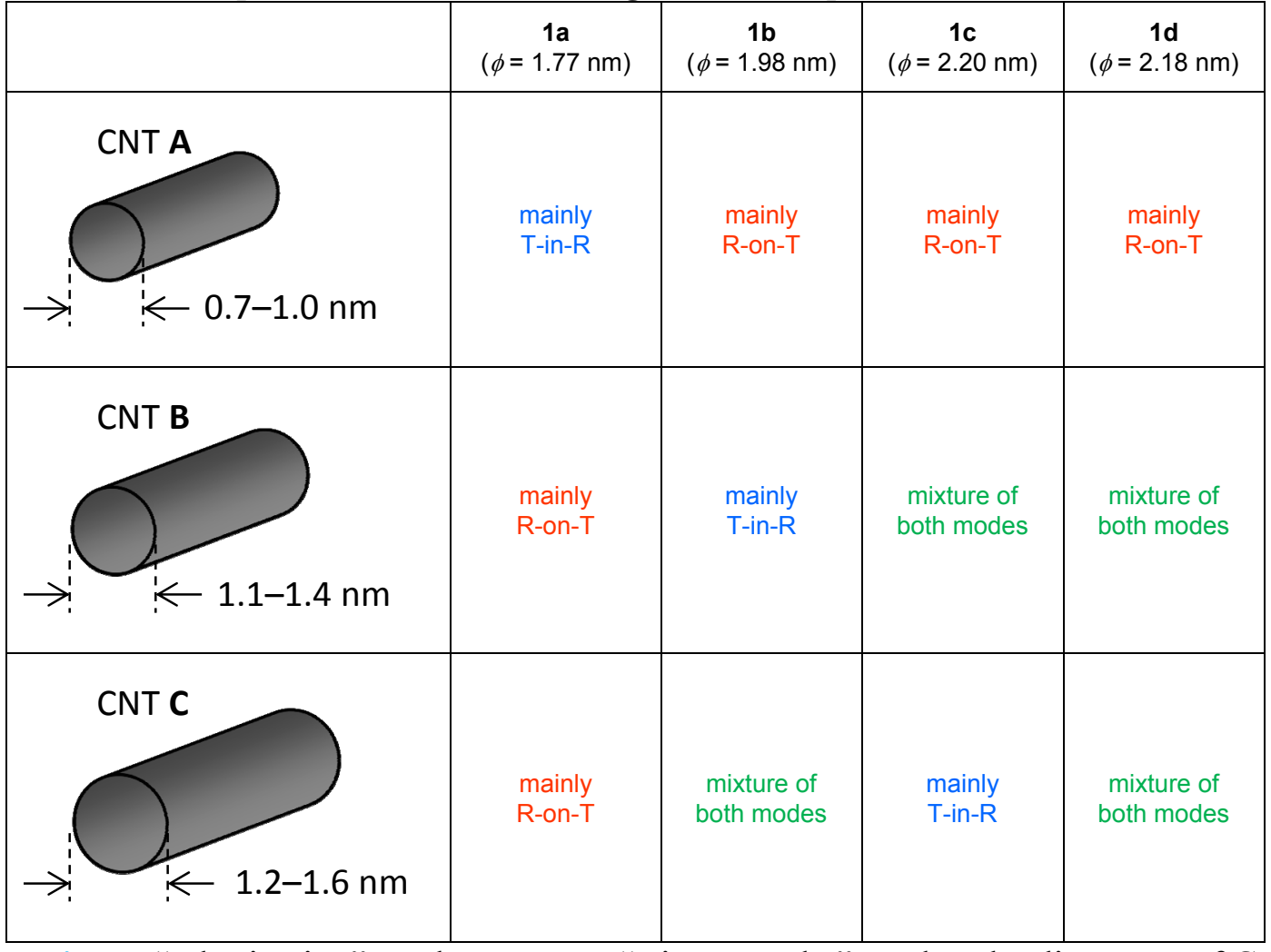

T-in-R: "tube-in-ring" mode, R-on-T: "Ring-on-tube" mode. The diameters of CPPAs complexed with CNTs were shown (see Figure 4)

\section{Conclusion}

We have developed a new method, the "ring toss" method, to prepare "tube-in-ring" supramolecular complexes of CNTs. $\pi$-Conjugated carbon nanorings, [8]-, [9]-, and [10]CPPAs, efficiently interact with CNTs to form CNT@CPPA complexes. We found that the "tube-in-ring"-type complexation proceeded smoothly when CNTs tightly fit in the cavities of CPPAs through convex-concave interaction. We assume that the "tube-in-ring" complexation of anthracene-containing CPPAs with curved $\pi$-conjugated molecules, with high association constants, is initiated by the "ball-on-bowl" and/or "ring-in-ring" interaction with the CNT terminus to form CNT@CPPA complexes. TGA showed that "ring-on-tube"type and "tube-in-ring"-type complexes could be distinguished by temperatures of weight loss. 


\section{WILEY-VCH}

RBM upfield shifts in Raman spectroscopy and TEM images suggest the formation of "tubein-ring"-type CNT@CPPA complexes. In SPM measurements, the "tube-in-ring"-type and "ring-on-tube"-type complexations are distinguishable by height profile analysis. This "ring toss" method for the preparation of functionalized CNTs will find application in electronic and biomedical applications.

\section{Experimental Section}

Materials: The preparation of CPPAs is summarized in the Supporting Information. Single walled carbon nanotubes were purchased from Sigma-Aldrich (USA), KH Chemicals CO., Ltd. (Korea), and Meijo Nano Carbon Co., Ltd. (Japan). CNTs: carbon nanotube, singlewalled $((6,5)$ chirality, carbon as SWCNT $>93 \%$, average diameter: 0.7-0.9 nm, CNT A), KH SWCNT HP (carbon purity $>80 \%$, diameter: $1.0-1.3 \mathrm{~nm}$, CNT B), and Meijo eDIPs EC1.5-P (purity: $>99 \%$, diameter: $1.5 \pm 0.5 \mathrm{~nm}, \mathrm{CNT} \mathbf{C}$ ). The diameters are representative values provided by vendors. All CNTs were purified under sonication in hydrogen peroxide before use (see the Supporting Information).

Methods: Samples were dropped onto a TEM copper grid covered with a carbon film (NPC15 STEM Cu150P, 167 mesh, Okenshoji Co., Ltd., Japan) and dried for $3 \mathrm{~h}$. Highresolution TEM measurements were performed using a JEOL JEM-2100F microscope equipped with double JEOL Delta spherical aberration correctors and a Gatan UltraScan 1000XP CCD camera at an electron accelerating voltage of $60 \mathrm{kV}^{[22]} \mathrm{TEM}$ image simulation was performed using SimulaTEM software. ${ }^{[23]}$

For SPM observation, a mixture of CNT@CPPA complexes in DMF or ethanol were spincoated on a freshly-prepared mica plate. The observation of complexes with SPM were conducted with SPM-9700HT (Shimadzu Co., Japan). 


\section{WILEY-VCH}

TGA (flow: air $\left(50 \mathrm{~mL} \mathrm{~min}^{-1}\right)$, scan rate: $\left.10{ }^{\circ} \mathrm{C} \mathrm{min}^{-1}\right)$ of CNT@CPPA complexes 2 were carried out by using TG-60 (Shimadzu Co., Japan).

Raman spectroscopy of CNT@CPPA complexes were conducted by LabRAM HR Evolution (HORIBA, Ltd., $\lambda_{\mathrm{ex}}=488 \mathrm{~nm}$ ) and LabRAM ARAMIS (HORIBA, Ltd., $\lambda_{\mathrm{ex}}=532$ $\mathrm{nm}$ ). All measurements were carried out at least twice to check the reproducibility.

Complexation of CNT with CPPA: General procedure for complexation of CNT with CPPA is as follows, purified CNT $(0.2 \mathrm{mg})$ was dispersed in ODCB $(0.4 \mathrm{~mL})$ and sonicated for $1 \mathrm{~h}$. To the resulting mixture was added CPPAs $(0.8 \mathrm{mg})$ in ODCB $(4 \mathrm{~mL})$ at room temperature. After gently stirring for $18 \mathrm{~h}$ in the dark, CNT@CPPA complex $(\sim 0.2 \mathrm{mg})$ was collected by filtration using filter paper (pore size: $1 \mu \mathrm{m}$ ), thoroughly washed with ODCB, and dried under reduced pressure. The filtrate was diluted in $20 \mathrm{~mL}$ volumetric flask and the absorbance of CPPA recovered was measured by UV-vis spectrometer (UH5300, Hitachi, High-Tech Science Co., Japan). For comparison, the incubation of CPPA in ODCB without CNT was similarly prepared. The quantification experiments were carried out at least five times independently to check their reproducibility. Representative data were shown in Figure S6 in the Supporting Information.

Theoretical calculation of CPPAs: The structures of CPPAs 1a-d were optimized by DFT calculation (Gaussian09, ${ }^{[24]}$ B3LYP/6-31G(d) level ${ }^{[17]}$ ). For 1c and 1d, butoxy and butyl groups were replaced to methoxy and methyl groups, respectively. The energy-minimized structures were visualized by Mercury software (Mercury 3.6) provided from Cambridge Crystallographic Data Centre (CCDC). TD-DFT calculation was carried out at CAMB3LYP/6-31G(d) level ${ }^{[25]}$. The frontier orbitals of CPPAs 1a and $\mathbf{1 b}$ were visualized by GaussView 4.1 and summarized in Figure S3 and S4 in the Supporting Information.

Theoretical calculation of CNT@CPPA complexes: CNT@CPPA complexes were optimized by force field calculation with MMFF94 ${ }^{[19]}$ or Forcite in Materials Studio 2017 R2 software (BIOVIA), and DFT calculation at B3LYP/6-31G(d) level. ${ }^{[17]}$ Molecular 


\section{WILEY-VCH}

coordinates of CNTs were prepared by TubeGen, ${ }^{[26]}$ and the terminal carbons were capped with hydrogen atoms. Free energy difference between before and after complexation are summarized in the Supporting Information.

\section{Supporting Information}

Supporting Information is available from the Wiley Online Library or from the author.

\section{Acknowledgements}

This work was supported by a Grant-in-Aid for Young Scientists (A) (No. 26708024), a Grant-in-Aid for Scientific Research on Innovative Areas "New Polymeric Materials Based on Element-Blocks (No. 2401)" (15H00737 and 15H00739) of The Ministry of Education, Culture, Sports, Science, and Technology, Japan. We acknowledge Ms. Tomoko Numata in Horiba, Ltd. for the measurement of Raman spectrometry. We are grateful to Kyoto Integrated Science \& Technology Bio-Analysis Center for their supports to measure Raman spectrometry. We thank Mr. Masao Matsuda and Ms. Shiho Moriguchi in Shimadzu Co. for the measurement of SPM. Computation time was provided by the SuperComputer System, Institute for Chemical Research, Kyoto University.

Received: ((will be filled in by the editorial staff))

Revised: ((will be filled in by the editorial staff)) Published online: ((will be filled in by the editorial staff))

\section{References}

[1] For selected recent reviews, see: a) L. Brownlie, J. Shapter, Carbon 2018, 126, 257; b)

M. D. Yadav, K. Dasgupta, A. W. Patwardhan, J. B. Joshi, Int. Eng. Chem. Res. 2017, 56, 12407; c) J. Lefebvre, J. Ding, Z. Li, P. Finnie, G. Lopinski, P. R. L. Malenfant, Acc. Chem. Res. 2017, 50, 2479; d) J. L. Blackburn, ACS Energy Lett. 2017, 2, 1598; e) S. N. Habisreutinger, R. J. Nicholas, H. J. Snaith, Adv. Energy Mater. 2017, 1601839; f) L. Yu, C. Shearer, J. Shapter, Chem. Rev. 2016, 116, 13413; g) D. R. Barbero, S. D. Stranks, Adv. Mater. 2016, 28, 9668; h) C. Farrera, F. T. Andón, N. Feliu, ACS Nano 2017, 11, 10637; i) M. Hernández-Rivera, N. G. Zaibaq, L. J. Wilson, Biomaterials 2016, 101, 229; j) R. Alshehri, A. M. Ilyas, A. Hasan, A. Arnaout, F. Ahmed, A. Memic, J. Med. Chem. 2016, 59, 8149.

[2] a) M. Kawamoto, P. He, Y. Ito, Adv. Mater. 2017, 29, 1602423; b) B. Dinesh, A. Bianco, C. M. Ménard-Moyon, Nanoscale 2016, 8, 18596; c) P. Singh, S. Campidelli, S. 


\section{WILEY-VCH}

Giodani, D. Bonifazi, A. Bianco, M. Prato, Chem. Soc. Rev. 2009, 38, 2214; d) T. Umeyama, J. Baek, Y. Sato, K. Suenaga, F. Abou-Chahine, N. V. Tkachenko, H. Lemmetyinen, H. Imahori, Nat. Commun. 2015, 6, 7732.

[3] B. W. Smith, M. Monthioux, D. E. Luzzi, Nature 1998, 396, 323.

[4] A. N. Khlobystov, ACS Nano 2011, 5, 9306.

[5] For selected recent reviews, see: a) S. Bhattacharya, S. K. Samanta, Chem. Rev. 2016, 116, 11967; b) S. Mallakpour, S. Soltanian, $R S C A d v$. 2016, 6, 109916; c) H. Wang, Z. Bao, Nano Today 2015, 10, 737; d) E. M. Pérez, N. Martín, Chem. Soc. Rev. 2015, 44, 6425. For selected recent examples, see: e) J. Calbo, A. López-Moreno, A. de Juan, J. Comer, E. Ortí, E. M. Pérez, Chem. Eur. J. 2017, 23, 12909; f) P.-I. Wang, C.-Y. Tsai, Y.-J. Hsiao, J.-C. Jiang, D.-J. Liaw, Macromolecules 2016, 49, 8520; g) G. Depotter, J.-H. Olimier, M. G. Glesner, P. Deria, Y. Bai, G. Bullard, A. S. Kumbhar, M. J. Therien, K. Clays, Chem. Commun. 2016, 52, 12206; h) J.-H. Olivier, J. Park, P. Deria, J. Rawson, Y. Bai, A. S. Kumbhar, M. J. Therien, Angew. Chem. Int. Ed. 2015, 54, 8133; i) H. Yang, V. Bezugly, J. Kunstmann, A. Filoramo, G. Cuniberti, ACS Nano 2015, 9, 9012; j) J. López-Andarias, J. L. López, C. Atienza, F. G. Brunetti, C. Romero-Nieto, D. M. Guldi, N. Martín, Nat. Commun. 2014, 5, 3762.

[6] A. de Juan, Y. Pouillon, L. Ruiz-González, A. Torres-Pardo, S. Casado, N. Martín, Á. Rubio, E. M. Pérez, Angew. Chem. Int. Ed. 2014, 53, 5394.

[7] a) E. M. Pérez, Chem. Eur. J. 2017, 23, 12681; b) A. López-Moreno, E. M. Pérez, Chem. Commun. 2015, 51, 5421; c) A. de Juan, M. M. Bernal, E. M. Pérez, ChemPlusChem 2015, 80, 1153; d) E. Martínez-Periňán, A. de Juan, Y. Pouillon, C. Schieri, V. Strauss, N. Martín, Á. Rubio, E. Lorenzo, E. M. Pérez, Nanoscale 2016, 8, 9254; e) A. López-Moreno, B. Nieto-Ortega, M. Moffa, A. de Juan, M. M. Bernal, J. P. Fernández-Blázquez, J. J. Vilatela, D. Pisignano, E. M. Pérez, ACS Nano 2016, 10, 8012; f) S. Leret, Y. Pouillon, S. Casado, C. Navio, Á. Rubio, E. M. Pérez, Chem. Sci. 2017, 8, 1927.

[8] J. F. Stoddart, Chem. Soc. Rev. 2009, 38, 1802. 


\section{WILEY-VCH}

[9] K. Momma, F. Izumi, J. Appl. Crystallogr. 2011, 44, 1272.

[10] a) T. Kawase, Synlett 2007, 2609; b) T. Kawase, H. Kurata, Chem. Rev. 2006, 106, 5250.

[11] a) M. Petrukhina, L. T. Scott, Fragments of Fullerenes and Carbon Nanotubes: Designed Synthesis, Unusual Reactions, and Coordination Chemistry. John Wiley \& Sons, Inc. 2011, Chap. 11, 291; b) B. Steinberg, L. T. Scott, Angew. Chem. Int. Ed. 2009, 48, 5400; c) S. E. Lewis, Chem. Soc. Rev. 2015, 44, 2221; d) M. R. Golder, R. S. Jasti, Acc. Chem. Res. 2015, 48, 557; e) S. Yamago, E. Kayahara, T. Iwamoto, Chem. Rec. 2014, 14, 84; f) H. Omachi, Y. Segawa, K. Itami, Acc. Chem. Res. 2012, 45, 1378.

[12] T. Kawase, K. Tanaka, N. Fujiwara, H. R. Darabi, M. Oda, Angew. Chem. Int. Ed. 2003, 42, 1624.

[13] K. Miki, T. Matsushita, Y. Inoue, Y. Senda, T. Kowada, K. Ohe, Chem. Commun. 2013, 49, 9092.

[14] a) T. Kawase, K. Tanaka, N. Shino, Y. Seirai, M. Oda, Angew. Chem. Int. Ed. 2004, 43, 1722; b) T. Kawase, Y. Nishiyama, T. Nakamura, T. Ebi, K. Matsumoto, H. Kurata, M. Oda, Angew. Chem. Int. Ed. 2007, 46, 1086.

[15] S. Hashimoto, T. Iwamoto, D. Kurachi, E. Kayahara, S. Yamago, ChemPlusChem 2017, 82, 1015.

[16] T. Iwamoto, Y. Watanabe, Y. Sakamoto, T. Suzuki, S. Yamago, J. Am. Chem. Soc. 2011, 133, 8354 .

[17] a) A. D. Becke, J. Chem. Phys. 1993, 98, 5648; b) C. Lee, W. Yang, R. G. Parr, Phys. Rev. $B$ 1988, 37, 785.

[18] C. Zhang, X. Chen, Ultrason. Sonochem. 2017, 34, 19.

[19] T. A. Halgren, J. Comp. Chem. 1996, 17, 616.

[20] T. Kawase, N. Fujiwara, M. Tsutumi, M. Oda, Y. Maeda, T. Wakahara, T. Akasaka, Angew. Chem. Int. Ed. 2004, 43, 5060. 
[21] P. M. Ajayan, T. W. Ebbesen, T. Ichihashi, S. Iijima, K. Tanigaki, H. Hiura, Nature, $1993,362,522$.

[22] T. Sasaki, H. Sawada, F. Hosokawa, Y. Kohno, T. Tomita, T. Kaneyama, Y. Kondo, K. Kimoto, Y. Sato, K. Suenaga, J. Electron Microsc. 2010, 59, S7.

[23] A. Gómez-Rodríguez, L. M. Beltán-del-Río, R. Herrera-Becerra, Ultramicroscopy 2010, 110,95 .

[24] Gaussian 09, Revision B.01, M. J. Frisch, et al., Gaussian, Inc., Wallingford CT, USA (2010).

[25] T. Yanai, D. P. Tew, N. C. Handy, Chem. Phys. Lett. 2004, 393, 51.

[26] TubeGen 3.4 (web-interface, http://turin.nss.udel.edu/research/tubegenonline.html), J. T. Frey and D. J. Doren, University of Delaware, Newark DE, 2011. 


\section{WILEY-VCH}

The table of contents entry should be 50-60 words long, and the first phrase should be bold. The entry should be written in the present tense and impersonal style. The text should be different from the abstract text.

"Ring toss": Carbon nanotubes (CNTs), which tightly fit in the cavities of $\pi$-conjugated carbon nanorings, [8]-, [9]-, and [10]cycloparaphenyleneacetylenes (CPPAs), through convex-concave interaction, efficiently afford "tube-in-ring"-type CNT@CPPA complexes.

\section{Keyword}

carbon nanotube, carbon nanoring, supramolecular, rotaxane

Figures 2, 3, 7, 9, out in full.

Koji Miki, Kenzo Saiki, Tomokazu Umeyama, Jinseok Baek, Takeru Noda, Hiroshi Imahori, Yuta Sato, Kazu Suenaga, and Kouichi Ohe

Title ((no stars))

Unique Tube-Ring Interactions: Complexation of Single-Walled Carbon Nanotubes with Cycloparaphenyleneacetylenes

ToC figure ((Please choose one size: $55 \mathrm{~mm}$ broad $\times 50 \mathrm{~mm}$ high or $110 \mathrm{~mm}$ broad $\times 20 \mathrm{~mm}$ high. Please do not use any other dimensions))

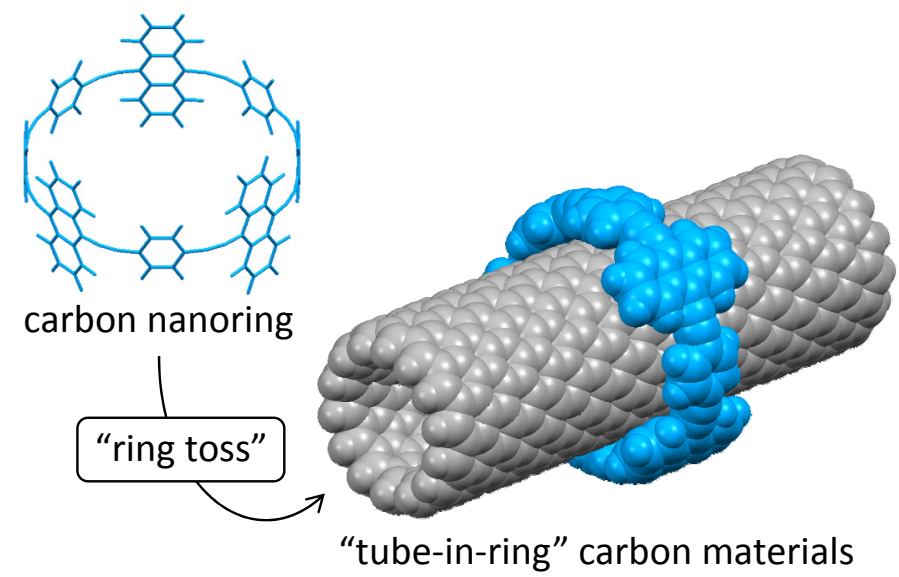


Copyright WILEY-VCH Verlag GmbH \& Co. KGaA, 69469 Weinheim, Germany, 2016.

\section{Supporting Information}

Unique Tube-Ring Interactions: Complexation with Single-Walled Carbon Nanotubes with Cycloparaphenyleneacetylenes

Koji Miki, * Kenzo Saiki, Tomokazu Umeyama, Jinseok Baek, Takeru Noda, Hiroshi Imahori, Yuta Sato, Kazu Suenaga, and Kouichi Ohe* 


\section{WILEY-VCH}

-INDEX-

1. Materials and methods

3.4. Complexation of CNT with CPPAs and quantification experiment 


\section{WILEY-VCH}

\section{Materials and methods}

Copper(I) iodide (CuI), ammonium chloride $\left(\mathrm{NH}_{4} \mathrm{Cl}\right)$, [tetrakis(triphenylphosphine)palladium $(0)$ ] $\left(\mathrm{Pd}\left(\mathrm{PPh}_{3}\right)_{4}\right)$, magnesium sulfate $\left(\mathrm{MgSO}_{4}\right)$, ethyl acetate (EtOAc), hexane, potassium peroxodisulfate $\left(\mathrm{K}_{2} \mathrm{~S}_{2} \mathrm{O}_{8}\right)$, diisopropylamine, o-dichlorobenznene (ODCB), 30\% $\mathrm{H}_{2} \mathrm{O}_{2}$ aqueous solution, and carbon disulfide $\left(\mathrm{CS}_{2}\right)$ were purchased from nacalai tesque (Japan). 4-Iodobenzaldehyde, diethyl chlorophosphate, lithium bis(trimethylsilyl)amide (1.3 M in THF), and trimethylsilylacetylene (TMSA) were purchased from Tokyo Chemical Industry (TCI) Co., Ltd. (Japan). Triethylamine $\left(\mathrm{Et}_{3} \mathrm{~N}\right)$, tetrahydrofuran (THF, super dehydrated), dichloromethane $\left(\mathrm{CH}_{2} \mathrm{Cl}_{2}\right.$, super dehydrated), chloroform $\left(\mathrm{CHCl}_{3}\right)$, iodine $\left(\mathrm{I}_{2}\right)$, sulfuric acid $\left(\mathrm{H}_{2} \mathrm{SO}_{4}\right)$, trifluoroacetic acid (TFA), tin(II) chloride dehydrate $\left(\mathrm{SnCl}_{2} \cdot 2 \mathrm{H}_{2} \mathrm{O}\right)$, and hydrochloric acid $(\mathrm{HCl})$ were purchased from Wako Pure Chemicals Inc. (Japan). $n$-Butyllithium (1.6 $\mathrm{M}$ in hexane) was purchased from Rockwood Lithium Japan K.K. (Japan). $\quad t$-Buthyldimethylsilyl chloride was purchased from fluorochem Ltd. (UK). 1,4Diiodobenzene was purchased from Alfa Aesar (USA). Tetra- $n$-butylammonium fluoride (1.0 M in THF) was purchased from Sigma-Aldrich (USA). $p$-Bromoiodobenzene was purchased from Apollo Scientific (UK). Potassium carbonate $\left(\mathrm{K}_{2} \mathrm{CO}_{3}\right)$ was purchased from Kishida Chemical Co., Ltd. (Japan). Organic solvents were distilled before use except THF (super dehydrated) and $\mathrm{CH}_{2} \mathrm{Cl}_{2}$ (super dehydrated).

Silica gel ( $\mathrm{SiO}_{2}, 230-400$ mesh) for column chromatography was purchased from Silicycle (Canada).

If necessary, compounds including CPPAs were purified by recycling gel permeation chromatography (GPC) apparatuses, LaboACE LC-5060 (recycling preparative HPLC, Japan Analytical Industry Co., Ltd., Japan) equipped with two preparative columns (JAIGEL-HR2 x 2) by using $\mathrm{CHCl}_{3}$ as an eluent $\left(10 \mathrm{~mL} \mathrm{~min}^{-1}\right)$ and LC-908 (recycling preparative HPLC, Japan Analytical Industry Co., Ltd., Japan) equipped with two preparative columns (JAIGEL-1H and JAIGEL-2H) by using $\mathrm{CHCl}_{3}$ as an eluent $\left(3.8 \mathrm{~mL} \mathrm{~min}{ }^{-1}\right)$ or two preparative columns (JAIGEL 1H-40 and JAIGEL $2 \mathrm{H}-40)$ by using $\mathrm{CHCl}_{3}$ as an eluent $\left(14 \mathrm{~mL} \mathrm{~min}^{-1}\right)$. 


\section{WILEY-VCH}

\subsection{Synthesis of building blocks of CPPA precursors}

The syntheses of building blocks of [8]-, [9]-, and [10]CPPA precursors were summarized in Schemes S1 and S2.

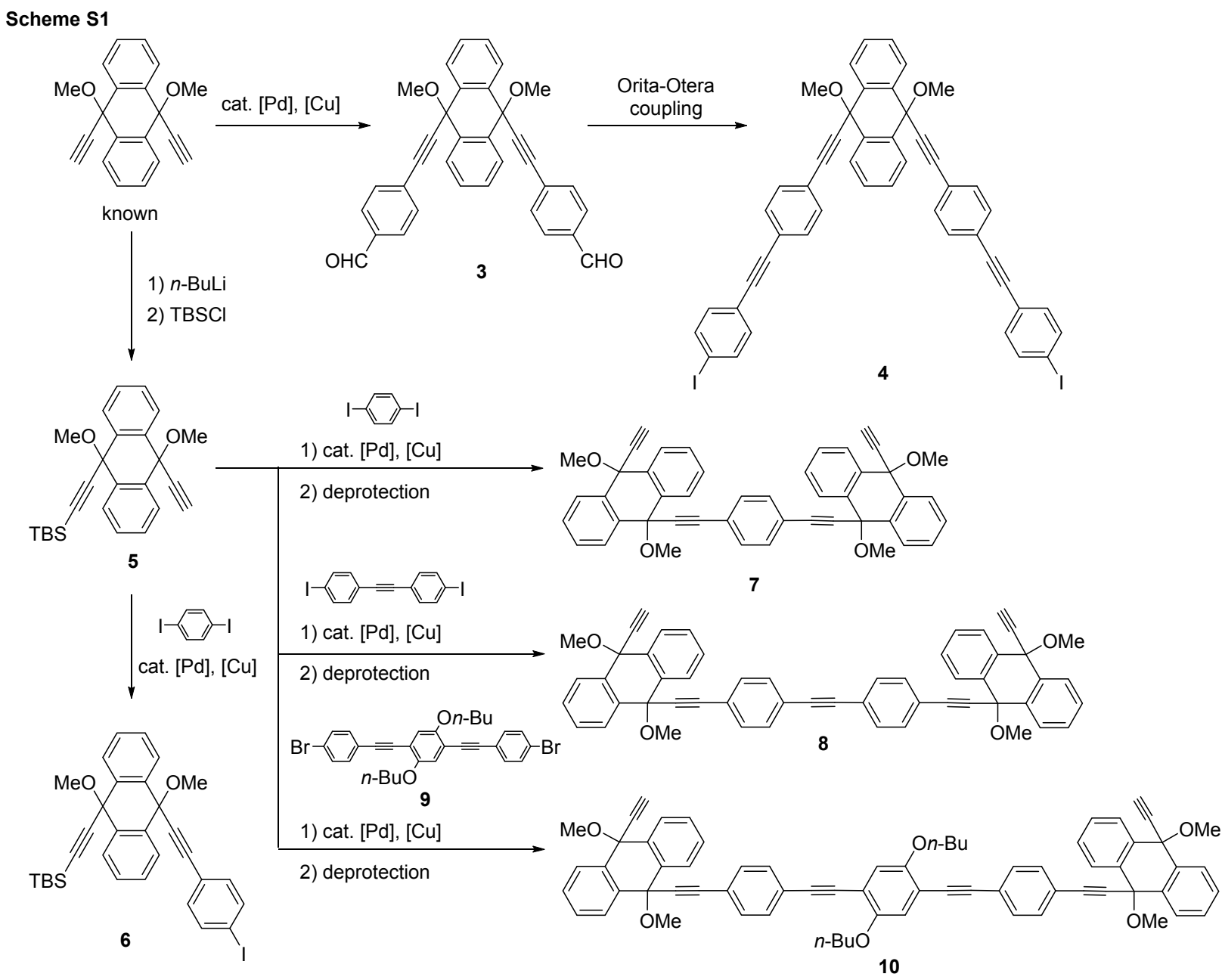

\section{Scheme S2}
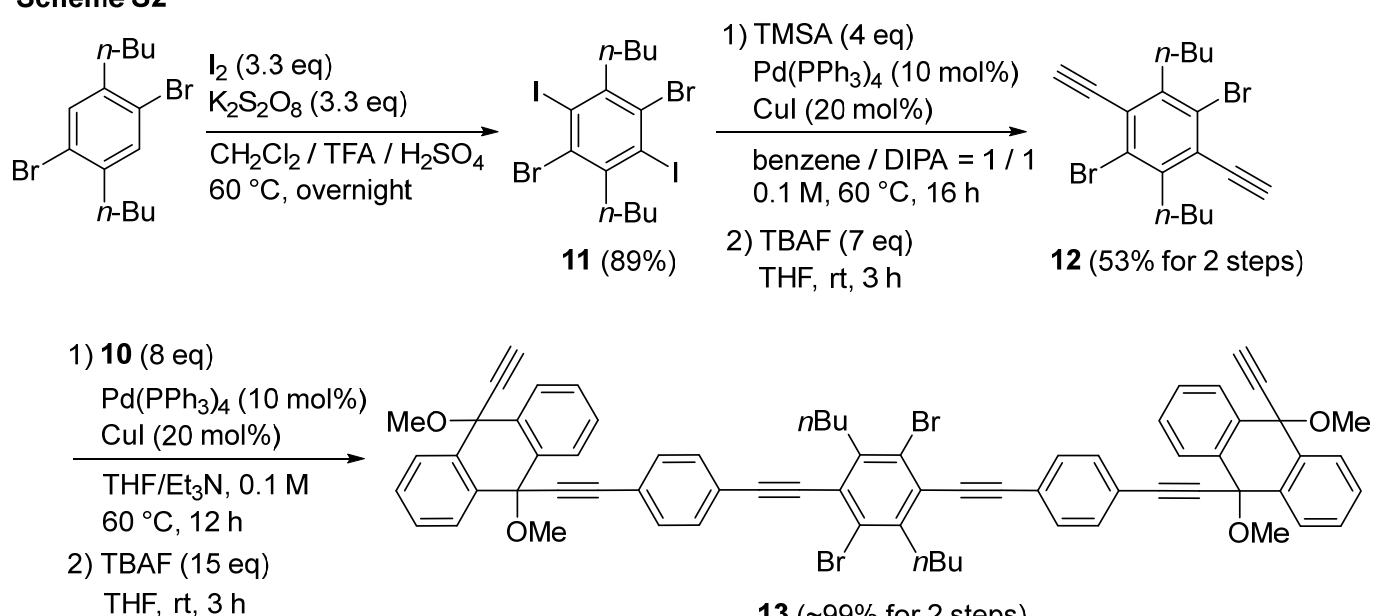


\section{WILEY-VCH}

\section{Compound 3}

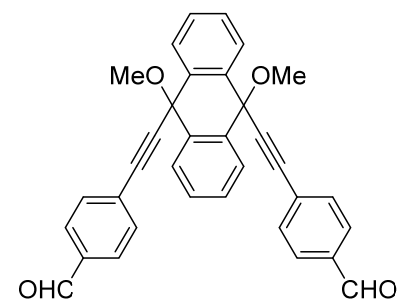

A solution of 9,10-diethynyl-9,10-dimethoxy-9,10-dihydroanthracene ${ }^{1}$ $(1.2 \mathrm{~g}, 4.0 \mathrm{mmol})$ and 4-iodobenzaldehyde $(2.1 \mathrm{~g}, 9.0 \mathrm{mmol})$ in $\mathrm{Et}_{3} \mathrm{~N}(8$ $\mathrm{mL})$ and THF $(32 \mathrm{~mL})$ was deoxygenized by freeze-pump-thaw cycling. To this solution was added $\mathrm{CuI}(0.15 \mathrm{~g}, 0.80 \mathrm{mmol})$ and $\mathrm{Pd}\left(\mathrm{PPh}_{3}\right)_{4}(0.69 \mathrm{~g}$, $0.60 \mathrm{mmol}$ ) at room temperature. After stirring for $18 \mathrm{~h}$ at $60{ }^{\circ} \mathrm{C}$, the resulting mixture was washed with sat. $\mathrm{NH}_{4} \mathrm{Cl}$ aqueous solution $(20 \mathrm{~mL})$. The aqueous layer was extracted with $\mathrm{CHCl}_{3}(15 \mathrm{~mL} \times 2)$ and the combined organic solution was dried over $\mathrm{MgSO}_{4}$. Organic solvents were removed under reduced pressure and the residue was purified by GPC to afford 3 (1.4 g, $2.9 \mathrm{mmol}, 73 \%)$ as a pale yellow solid. mp $151.0-153.2{ }^{\circ} \mathrm{C}$ (dec); IR (ATR) 2927, $2222(\mathrm{C} \equiv \mathrm{C}), 1703$ (C=O), 1601, 1563, 1450, 1302, 1262, 1205, 1165, 1065, 1023, 907, 828, 786, 764, $704 \mathrm{~cm}^{-1} ;{ }^{1} \mathrm{H}$ NMR $\left(300 \mathrm{MHz}, \mathrm{CDCl}_{3}, 25^{\circ} \mathrm{C}\right) \delta 3.03(\mathrm{~s}, 6 \mathrm{H}), 7.52-7.57(\mathrm{~m}, 8 \mathrm{H}), 7.76(\mathrm{~d}, J=8.4$ $\mathrm{Hz}, 8 \mathrm{H}), 8.05(\mathrm{dd}, J=2.6,9.2 \mathrm{~Hz}, 4 \mathrm{H}), 9.97(\mathrm{~s}, 2 \mathrm{H}) ;{ }^{13} \mathrm{C} \mathrm{NMR}\left(75 \mathrm{MHz}, \mathrm{CDCl}_{3}, 25{ }^{\circ} \mathrm{C}\right) \delta 51.6,72.8$, 86.3, 94.5, 128.0, 128.4, 128.6, 129.3, 132.3, 135.1, 135.7, 191.3. HRMS (FAB) calcd for $\mathrm{C}_{33} \mathrm{H}_{21} \mathrm{O}_{3}$ $\left([\mathrm{M}-\mathrm{OMe}]^{+}\right):$465.1491. Found 465.1500.

\section{Compound 4}

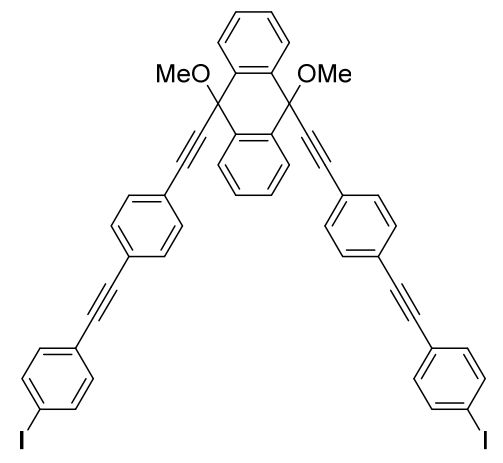

Diiodide 4 was synthesized by utilizing Orita-Otera method. ${ }^{2}$ To a solution of $\mathbf{3}(0.51 \mathrm{~g}, 1.0 \mathrm{mmol})$, 4-iodobenzylphenyl sulfone ${ }^{2}(0.78 \mathrm{~g}$, $2.2 \mathrm{mmol})$, and diethyl chlorophosphate $(0.33 \mathrm{~mL}, 2.3 \mathrm{mmol})$ in THF $(20 \mathrm{~mL})$ was slowly added lithium bis(trimethylsilyl)amide (1.3 $\mathrm{M}$ in THF, $7.7 \mathrm{~mL}, 10 \mathrm{mmol}$ ) at $0{ }^{\circ} \mathrm{C}$. After stirring for $1 \mathrm{~h}$ at $0{ }^{\circ} \mathrm{C}$ and for $17 \mathrm{~h}$ at room temperature, the resulting mixture was washed with sat. $\mathrm{NH}_{4} \mathrm{Cl}$ aqueous solution $(20 \mathrm{~mL})$. The aqueous layer was extracted with EtOAc $(15 \mathrm{~mL} \times 2)$ and the combined organic solution was dried over $\mathrm{MgSO}_{4}$. Organic solvents were removed under reduced pressure and the yellow residue was recrystallized from $\mathrm{CHCl}_{3}$ and hexane to afford $4(0.45 \mathrm{~g}, 0.50 \mathrm{mmol}, 50 \%)$ as a pale yellow solid. $\mathrm{mp}$ 241.2-243.5 ${ }^{\circ} \mathrm{C}$ (dec); IR (KBr) 2828, 2818, 2218 (C $\left.=\mathrm{C}\right), 1508,1234,1085,1007,962,908,838,822$, 754, $521 \mathrm{~cm}^{-1} ;{ }^{1} \mathrm{H}$ NMR $\left(300 \mathrm{MHz}, \mathrm{CDCl}_{3}, 25^{\circ} \mathrm{C}\right) \delta 3.04(\mathrm{~s}, 6 \mathrm{H}), 7.21(\mathrm{~d}, J=8.0 \mathrm{~Hz}, 4 \mathrm{H}), 7.37(\mathrm{~d}, J=$ $2.2 \mathrm{~Hz}, 8 \mathrm{H}), 7.53(\mathrm{dd}, J=3.7,5.9 \mathrm{~Hz}, 4 \mathrm{H}), 7.66(\mathrm{~d}, J=8.4 \mathrm{~Hz}, 4 \mathrm{H}), 8.05(\mathrm{dd}, J=3.3,5.9 \mathrm{~Hz}, 4 \mathrm{H})$; ${ }^{13} \mathrm{C}$ NMR $\left(100 \mathrm{MHz}, \mathrm{CDCl}_{3}, 25{ }^{\circ} \mathrm{C}\right) \delta 51.6,73.0,87.1,90.3,90.3,92.4,94.4,122.4,122.5,123.0$, 128.4, 129.1, 131.3, 131.7, 133.0, 135.4, 137.5. HRMS (FAB) calcd for $\mathrm{C}_{48} \mathrm{H}_{31} \mathrm{I}_{2} \mathrm{O}_{2}\left(\mathrm{M}+\mathrm{H}^{+}\right)$: 893.0413. Found 893.0388. 


\section{WILEY-VCH}

\section{Compound 5}

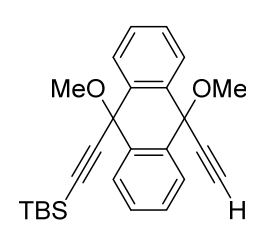

To a solution of 9,10-diethynyl-9,10-dimethoxy-9,10-dihydroanthracene ${ }^{1}(1.7 \mathrm{~g}$, $6.0 \mathrm{mmol})$ in THF $(80 \mathrm{~mL})$ was slowly added $n$-BuLi (1.6 M in hexane, $4.5 \mathrm{~mL}, 7.2$ mmol) at $-78{ }^{\circ} \mathrm{C}$. After stirring for $1.5 \mathrm{~h}$ at $-78{ }^{\circ} \mathrm{C}$, to this mixture was added $t$ butyldimethylsilyl chloride $(1.8 \mathrm{~g}, 1.2 \mathrm{mmol})$ at $0{ }^{\circ} \mathrm{C}$. After stirring for $1 \mathrm{~h}$ at $0{ }^{\circ} \mathrm{C}$ and for $14 \mathrm{~h}$ at room temperature, the resulting mixture was washed with water $(20 \mathrm{~mL})$. The aqueous layer was extracted with $\mathrm{CH}_{2} \mathrm{Cl}_{2}(15 \mathrm{~mL} \times 2)$ and the combined organic solution was dried over $\mathrm{MgSO}_{4}$. Organic solvents were removed under reduced pressure and the yellow residue was purified by GPC to afford 5 (1.1 g, $2.8 \mathrm{mmol}, 46 \%)$ as a yellow solid. mp 100.0-100.3 ${ }^{\circ} \mathrm{C}(\mathrm{dec})$; IR (ATR) 3241, 2948,

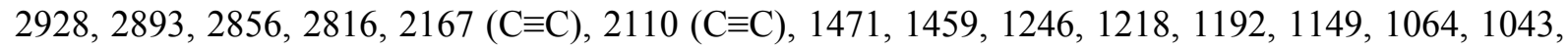
930, 904, 829, 813, 775, $765 \mathrm{~cm}^{-1} ;{ }^{1} \mathrm{H}$ NMR (300 MHz, $\left.\mathrm{CDCl}_{3}, 25{ }^{\circ} \mathrm{C}\right) \delta 0.14(\mathrm{~s}, 6 \mathrm{H}), 0.92(\mathrm{~s}, 9 \mathrm{H})$, $2.81(\mathrm{~s}, 1 \mathrm{H}), 3.00(\mathrm{~s}, 3 \mathrm{H}), 3.02(\mathrm{~s}, 3 \mathrm{H}), 7.47(\mathrm{dd}, J=3.3,5.9 \mathrm{~Hz}, 4 \mathrm{H}), 8.02(\mathrm{dd}, J=3.3,5.9 \mathrm{~Hz}, 4 \mathrm{H})$; ${ }^{13} \mathrm{C}$ NMR $\left(75 \mathrm{MHz}, \mathrm{CDCl}_{3}, 25{ }^{\circ} \mathrm{C}\right) \delta-4.7,16.7,26.1,51.7,51.7,73.3,73.7,76.2,84.2,92.0,105.8$, 128.6, 128.7, 128.8, 128.8, 135.1, 135.8. HRMS (FAB) calcd for $\mathrm{C}_{25} \mathrm{H}_{28} \mathrm{OSi}\left([\mathrm{M}-\mathrm{OMe}]^{+}\right): 371.1831$. Found 371.1830 .

\section{Compound 6}

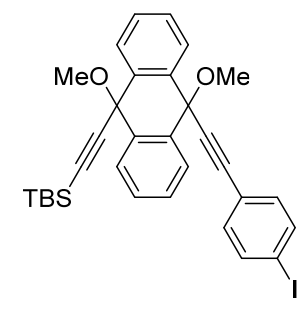

A solution of $5(0.44 \mathrm{~g}, 1.1 \mathrm{mmol})$ and 1,4-diiodobenzene $(1.2 \mathrm{~g}, 3.5 \mathrm{mmol})$ in $\mathrm{Et}_{3} \mathrm{~N}(2 \mathrm{~mL})$ and THF $(8 \mathrm{~mL})$ was deoxygenized by freeze-pump-thaw cycling. To the solution were added $\mathrm{CuI}(8.8 \mathrm{mg}, 46 \mu \mathrm{mol})$ and $\mathrm{Pd}\left(\mathrm{PPh}_{3}\right)_{4}(25 \mathrm{mg}, 22$ $\mu \mathrm{mol})$ at room temperature. After stirring at $60{ }^{\circ} \mathrm{C}$ for $8 \mathrm{~h}$, the resulting mixture was diluted with EtOAc $(10 \mathrm{~mL})$ and washed with sat. $\mathrm{NH}_{4} \mathrm{Cl}$ aqueous solution $(10 \mathrm{~mL})$. The organic solution was dried over $\mathrm{MgSO}_{4}$. Organic solvents were removed under reduced pressure and the brown residue was subjected to column chromatography on $\mathrm{SiO}_{2}$ with hexane/EtOAc $(\mathrm{v}: \mathrm{v}=10: 1)$ as an eluent to afford $6(0.46 \mathrm{~g}, 0.76 \mathrm{mmol}, 69 \%, \mathrm{rf}=0.45$ (hexane/EtOAc (v:v $=10: 1)$, starting material: $\mathrm{rf}=0.39)$ ) as a yellowish white solid. $\mathrm{mp} 87.1-$

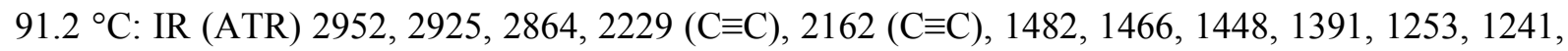
$1213,1084,1058,1006,950,842,817,770,754,735,692 \mathrm{~cm}^{-1} ;{ }^{1} \mathrm{H}$ NMR $\left(400 \mathrm{MHz}, \mathrm{CDCl}_{3}, 25^{\circ} \mathrm{C}\right) \delta$ 0.13 (s, 6H), 0.91 (s, 9H), 3.04 (s, 3H), 3.04 (s, 3H), 7.18 (d, $J=8.8 \mathrm{~Hz}, 2 \mathrm{H}), 7.47$ (dd, $J=3.3,5.9 \mathrm{~Hz}$, $4 \mathrm{H}), 7.64(\mathrm{~d}, J=8.8 \mathrm{~Hz}, 2 \mathrm{H}), 8.04(\mathrm{dd}, J=3.3,5.9 \mathrm{~Hz}, 4 \mathrm{H}) .{ }^{13} \mathrm{C} \mathrm{NMR}\left(100 \mathrm{MHz}, \mathrm{CDCl}_{3}, 25{ }^{\circ} \mathrm{C}\right) \delta-$ 4.7, 16.7, 26.1, 51.7, 51.8, 73.7, 73.9, 87.0, 91.4, 92.0, 94.5, 105.8, 122.0, 128.5, 128.7, 128.8, 128.8, 133.2, 135.4, 135.7, 137.4. HRMS (FAB) calcd for $\mathrm{C}_{31} \mathrm{H}_{30} \mathrm{OSiI}\left([\mathrm{M}-\mathrm{OMe}]^{+}\right)$: 573.1111. Found 573.1129 . 


\section{WILEY-VCH}

\section{Compounds 7 and 8}

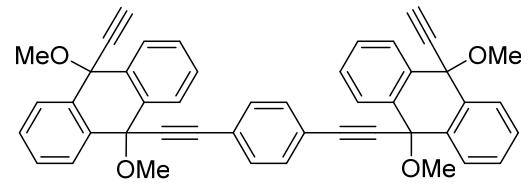

Compounds $\mathbf{7}$ and $\mathbf{8}$ were similarly synthesized from 1,4diiodobenzene and 1,2-bis(4-iodophenyl)ethyne, ${ }^{2}$ respectively. Synthetic procedure of $\mathbf{8}$ is shown below.

A solution of $5(1.9 \mathrm{~g}, 4.0 \mathrm{mmol})$ and 1,2-bis(4iodophenyl)ethyne $(0.90 \mathrm{~g}, 2.1 \mathrm{mmol})$ in $\mathrm{Et}_{3} \mathrm{~N}(4.2$

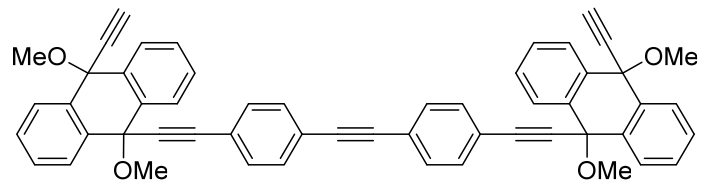
$\mathrm{mL})$ and THF $(17 \mathrm{~mL})$ was deoxygenized by freezepump-thaw cycling. To this solution was added $\mathrm{CuI}$ $(80 \mathrm{mg}, 0.42 \mathrm{mmol})$ and $\mathrm{Pd}\left(\mathrm{PPh}_{3}\right)_{4}(0.36 \mathrm{~g}, 0.31$ mmol) at room temperature. After stirring for $18 \mathrm{~h}$ at $60^{\circ} \mathrm{C}$, the resulting mixture was washed with sat. $\mathrm{NH}_{4} \mathrm{Cl}$ aqueous solution $(20 \mathrm{~mL})$. The aqueous layer was extracted with $\mathrm{CHCl}_{3}(15 \mathrm{~mL} \times 2)$ and the combined organic solution was dried over $\mathrm{MgSO}_{4}$. Organic solvents were removed under reduced pressure and the brown residue was purified by GPC to afford pentayne having TBS groups (1.4 g, 2.9 mmol, 73\%) as a brown solid. $\mathrm{mp} 212.0-213.3{ }^{\circ} \mathrm{C}$; $\mathrm{IR}(\mathrm{KBr})$ 2950, 2928, 2893, 2856, 2817, 2165 $(\mathrm{C} \equiv \mathrm{C}), 1512,1451,1361,1248,1145,1069,1051,929,906,828,761 \mathrm{~cm}^{-1} ;{ }^{1} \mathrm{H}$ NMR $(400 \mathrm{MHz}$, $\left.\mathrm{CDCl}_{3}, 25{ }^{\circ} \mathrm{C}\right) \delta 0.16(\mathrm{~s}, 12 \mathrm{H}), 0.94(\mathrm{~s}, 18 \mathrm{H}), 3.06(\mathrm{~s}, 6 \mathrm{H}), 3.08(\mathrm{~s}, 6 \mathrm{H}), 7.45-7.48(\mathrm{~m}, 8 \mathrm{H}), 7.49-7.52$ $(\mathrm{m}, 8 \mathrm{H}), 8.06-8.10(\mathrm{~m}, 8 \mathrm{H}) ;{ }^{13} \mathrm{C}$ NMR $\left(75 \mathrm{MHz}, \mathrm{CDCl}_{3}, 25{ }^{\circ} \mathrm{C}\right) \delta-4.7,16.9,26.0,51.7,51.8,73.7$, 73.9, 77.3, 87.6, 90.8, 91.9, 105.9, 122.5, 123.1, 128.8, 128.8, 128.8, 128.8, 131.4, 131.7, 135.5, 135.7 . HRMS (FAB) calcd for $\mathrm{C}_{65} \mathrm{H}_{63} \mathrm{O}_{3} \mathrm{Si}_{2}\left([\mathrm{M}-\mathrm{OMe}]^{+}\right)$: 947.4316. Found 947.4319.

To a solution of the pentayne $(0.23 \mathrm{~g}, 0.24 \mathrm{mmol})$ in $\mathrm{CH}_{2} \mathrm{Cl}_{2}(50 \mathrm{~mL})$ was added tetra- $n$ butylammonium fluoride (1 M in THF, $1.0 \mathrm{~mL}, 1.0 \mathrm{mmol}$ ) at room temperature. After stirring for $1 \mathrm{~h}$ at room temperature, the resulting mixture was washed with brine $(20 \mathrm{~mL})$. The aqueous layer was extracted with $\mathrm{CH}_{2} \mathrm{Cl}_{2}(15 \mathrm{~mL} \times 3)$ and the combined organic solution was dried over $\mathrm{MgSO}_{4}$. Organic solvents were removed under reduced pressure. The brown residue was washed with hexane and purified by short column chromatography on $\mathrm{SiO}_{2}$ using EtOAc as an eluent to afford $\mathbf{8}(0.18 \mathrm{~g}, 0.23$ mmol, quant.) as a brown solid. $\mathrm{mp} 114.7-116.4^{\circ} \mathrm{C}$; IR (KBr) 3286, 2930, 2819, 2222 (C $\left.\equiv \mathrm{C}\right), 2111$ $(\mathrm{C} \equiv \mathrm{C}), 1514,1448,1242,1076,1024,910,838,760 \mathrm{~cm}^{-1} ;{ }^{1} \mathrm{H}$ NMR (400 MHz, $\left.\mathrm{CDCl}_{3}, 25{ }^{\circ} \mathrm{C}\right) \delta 2.79$ (s, 2H), 2.98 (s, 6H), 3.01 (s, 6H), 7.39 (d, $J=8.8 \mathrm{~Hz}, 4 \mathrm{H}), 7.42$ (d, $J=8.8 \mathrm{~Hz}, 4 \mathrm{H}), 7.49-7.52$ (m, 8H), 8.00-8.05 (m, 8H); ${ }^{13} \mathrm{C}$ NMR (100 MHz, $\left.\mathrm{CDCl}_{3}, 25^{\circ} \mathrm{C}\right) \delta 51.5,51.6,72.5,73.1,75.8,84.7,87.1,90.8$, 92.2, 122.4 123.0, 128.4 128.5, 129.0, 129.1, 131.3, 131.7, 135.0, 135.4. HRMS (FAB) calcd for $\mathrm{C}_{54} \mathrm{H}_{38} \mathrm{O}_{4}\left(\mathrm{M}^{+}\right):$750.2770. Found 750.2745.

7: a pale yellow solid (95\% for two steps); mp 164.2-166.8 ${ }^{\circ} \mathrm{C}(\mathrm{dec})$; IR ( $\left.\mathrm{KBr}\right) 3239,2949,2823$, $2237(\mathrm{C} \equiv \mathrm{C}), 2110(\mathrm{C} \equiv \mathrm{C}), 1449,1285,1243,1075,952,913,838,758,692 \mathrm{~cm}^{-1}$; ${ }^{1} \mathrm{H}$ NMR $(400 \mathrm{MHz}$, $\left.\mathrm{CDCl}_{3}, 25{ }^{\circ} \mathrm{C}\right) \delta 2.78(\mathrm{~s}, 2 \mathrm{H}), 2.97(\mathrm{~s}, 6 \mathrm{H}), 2.99(\mathrm{~s}, 6 \mathrm{H}), 7.33(\mathrm{~s}, 4 \mathrm{H}), 7.48-7.51(\mathrm{~m}, 8 \mathrm{H}), 7.98-8.03(\mathrm{~m}$, $8 \mathrm{H}) ;{ }^{13} \mathrm{C} \mathrm{NMR}\left(100 \mathrm{MHz}, \mathrm{CDCl}_{3}, 25{ }^{\circ} \mathrm{C}\right) \delta 51.5,51.5,72.4,73.0,75.7,84.7,87.0,92.0,122.5,128.4$, 128.5, 129.0, 129.1, 131.5, 134.9, 135.4. HRMS (FAB) calcd for $\mathrm{C}_{45} \mathrm{H}_{31} \mathrm{O}_{3}\left(\left[\mathrm{M}-\mathrm{OMe}^{-}\right]^{+}\right): 619.2273$. Found 619.2267. 


\section{Compound 9}

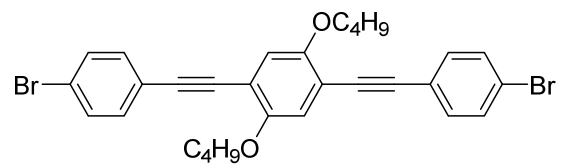

To a solution of 1,4-dibutoxy-2,5-diethynylbenznene ${ }^{3}(1.7 \mathrm{~g}$, $6.3 \mathrm{mmol})$ and $p$-bromoiodobenzene $(3.7 \mathrm{~g}, 13 \mathrm{mmol})$ in THF $(21 \mathrm{~mL})$ and $\mathrm{Et}_{3} \mathrm{~N}(7 \mathrm{~mL})$ were added $\mathrm{CuI}(0.12 \mathrm{~g}, 0.63 \mathrm{mmol})$ and $\mathrm{Pd}\left(\mathrm{PPh}_{3}\right)_{4}(0.36 \mathrm{~g}, 0.32 \mathrm{mmol})$ at room temperature. After stirring at $50{ }^{\circ} \mathrm{C}$ for $6 \mathrm{~h}$, the resulting mixture was concentrated under reduced pressure and the residue was diluted with $\mathrm{CH}_{2} \mathrm{Cl}_{2}(50 \mathrm{~mL})$. The organic solution was washed with sat. $\mathrm{NH}_{4} \mathrm{Cl}$ aqueous solution $(20 \mathrm{~mL})$ and water $(20 \mathrm{~mL})$. The aqueous layer was extracted with $\mathrm{CH}_{2} \mathrm{Cl}_{2}(15 \mathrm{~mL})$ and the combined organic solution was dried over $\mathrm{MgSO}_{4}$. Organic solvents were removed under reduced pressure and the brown residue was dispersed in hot hexane $(50 \mathrm{~mL})$. The mixture was cooled and filtered by glass filter. The yellowish brown solid was washed with hexane $(10 \mathrm{~mL} \times 2)$ and dried under reduced pressure to afford $9(3.2 \mathrm{~g})$ as a yellow solid. The filtrate was concentrated under vacuum and the residue was subjected to column chromatography on $\mathrm{SiO}_{2}$ to afford 9 (0.55 g) as a second crop. 9 (3.7 g, $\left.6.2 \mathrm{mmol}, 99 \%\right)$, mp 119.1$122.8^{\circ} \mathrm{C}$; IR (ATR) 3056, 2958, 2870, 2207 (C $\equiv \mathrm{C}$ ), 1499, 1480, 1464, 1434, 1390, 1278, 1215, 1095, 1067, 1009, 1000, 859, 823, 790, 741, $690 \mathrm{~cm}^{-1} ;{ }^{1} \mathrm{H}$ NMR (400 MHz, $\left.\mathrm{CDCl}_{3}, 25^{\circ} \mathrm{C}\right): \delta 1.00(\mathrm{t}, J=7.6$ $\mathrm{Hz}, 6 \mathrm{H}), 1.53-1.61(\mathrm{~m}, 4 \mathrm{H}), 1.80-1.86(\mathrm{~m}, 4 \mathrm{H}), 4.03(\mathrm{t}, J=6.6 \mathrm{~Hz}, 4 \mathrm{H}), 6.99$ (s, 2H), 7.38 (d, $J=8.4$ $\mathrm{Hz}, 4 \mathrm{H}), 7.49$ (d, $J=8.4 \mathrm{~Hz}, 4 \mathrm{H}) ;{ }^{13} \mathrm{C}$ NMR $\left(100 \mathrm{MHz}, \mathrm{CDCl}_{3}, 25{ }^{\circ} \mathrm{C}\right): \delta 13.9,19.3,31.3,69.3,87.1$, 93.8, 113.9, 116.8, 122.4, 122.5, 131.6, 132.9 153.6. HRMS (FAB) calcd for $\mathrm{C}_{30} \mathrm{H}_{28} \mathrm{Br}_{2} \mathrm{O}_{2}\left(\mathrm{M}^{+}\right)$: 578.0456. Found: 578.0457.

\section{Compound 10}

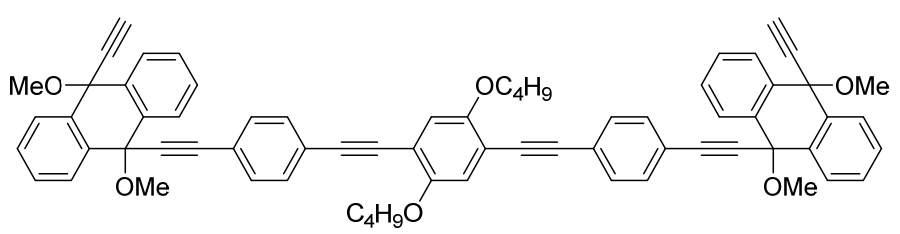

A solution of $9(0.28 \mathrm{~g}, 0.48 \mathrm{mmol})$ and $5(0.39 \mathrm{~g}, 0.97 \mathrm{mmol})$ in $\mathrm{Et}_{3} \mathrm{~N}(1 \mathrm{~mL})$ and THF $(4 \mathrm{~mL})$ was deoxygenized by freezepump-thaw cycling. To this solution was added $\mathrm{CuI}(7.3 \mathrm{mg}, 40 \mu \mathrm{mol})$ and $\mathrm{Pd}\left(\mathrm{PPh}_{3}\right)_{4}(22 \mathrm{mg}, 20 \mu \mathrm{mol})$ at room temperature. After stirring for $18 \mathrm{~h}$ at $60{ }^{\circ} \mathrm{C}$, the resulting mixture was diluted with $\mathrm{CH}_{2} \mathrm{Cl}_{2}(10 \mathrm{~mL})$ and washed with sat. $\mathrm{NH}_{4} \mathrm{Cl}$ aqueous solution $(20 \mathrm{~mL})$. The aqueous layer was extracted with $\mathrm{CH}_{2} \mathrm{Cl}_{2}(5 \mathrm{~mL} \times 2)$ and the combined organic solution was dried over $\mathrm{MgSO}_{4}$. Organic solvents were removed under reduced pressure and the brown residue was subjected to short column chromatography on $\mathrm{SiO}_{2}$ with hexane/EtOAc (v:v = 10:1 to $4: 1)$ as an eluent to afford the crude hexayne having TBS groups as a brown solid ( $>99 \%$ yield). ${ }^{1} \mathrm{H}$ NMR (300 MHz, $\left.\mathrm{CDCl}_{3}, 25{ }^{\circ} \mathrm{C}\right): \delta 0.14$ (s, 12H), 0.92 (s, $\left.18 \mathrm{H}\right), 1.00(\mathrm{t}, J=7.3 \mathrm{~Hz}, 6 \mathrm{H})$, 1.53-1.60 (m, 4H), 1.77-1.88 (m, 4H), $3.05(\mathrm{~s}, 6 \mathrm{H}), 3.07$ (s, 6H), $4.03(\mathrm{t}, J=6.4 \mathrm{~Hz}, 4 \mathrm{H}), 7.00(\mathrm{~s}, 2 \mathrm{H})$, 7.40-7.50 (m, 16H), 8.02-8.09 (m, 8H).

To a solution of the crude hexayne in THF $(10 \mathrm{~mL})$ was added tetra- $n$-butylammonium fluoride (1 $\mathrm{M}$ in THF, $1.0 \mathrm{~mL}, 1.0 \mathrm{mmol}$ ) at room temperature. After stirring for $1 \mathrm{~h}$ at room temperature, the resulting mixture was diluted with $\mathrm{CH}_{2} \mathrm{Cl}_{2}(10 \mathrm{~mL})$ and washed with brine $(20 \mathrm{~mL})$. The aqueous layer was extracted with $\mathrm{CH}_{2} \mathrm{Cl}_{2}(5 \mathrm{~mL} \times 2)$ and the combined organic solution was dried over $\mathrm{MgSO}_{4}$. 


\section{WILEY-VCH}

Organic solvents were removed under reduced pressure and the brown residue was subjected to short column chromatography on $\mathrm{SiO}_{2}$ with hexane/EtOAc ( $\mathrm{v}: \mathrm{v}=1: 0$ to $\left.4: 1\right)$ as an eluent to afford hexayne 10 (0.35 g, $0.35 \mathrm{mmol}, 74 \%$ for 2 steps) as a yellow solid. mp 85.1-96.4 ${ }^{\circ} \mathrm{C}$; IR (ATR) 3283, 2934, 2870, 2826, $2248(\mathrm{C} \equiv \mathrm{C}), 2210(\mathrm{C} \equiv \mathrm{C}), 2115(\mathrm{C} \equiv \mathrm{C}), 1515,1492,1475,1448,1416,1393,1277,1216$, 1081, 1023, 966, 907, 859, 837, 765, $729 \mathrm{~cm}^{-1} ;{ }^{1} \mathrm{H}$ NMR (400 MHz, $\left.\mathrm{CDCl}_{3}, 25{ }^{\circ} \mathrm{C}\right): \delta 0.98(\mathrm{t}, J=7.5$ $\mathrm{Hz}, 6 \mathrm{H}), 1.53-1.59$ (m, 4H), 1.77-1.85 (m, 4H), 2.79 (s, 2H), 2.99 (s, 6H), 3.01 (s, 6H), 4.02 (t, $J=6.2$ $\mathrm{Hz}, 4 \mathrm{H}), 6.98$ (s, 2H), 7.40-7.44 (m, 8H), 7.49-7.52 (m, 8H), 7.99-8.05 (m, 8H); ${ }^{13} \mathrm{C}$ NMR $(100 \mathrm{MHz}$, $\left.\mathrm{CDCl}_{3}, 25^{\circ} \mathrm{C}\right): \delta 13.9,19.3,31.3,51.6,51.6,69.3,72.6,73.2,75.8,84.7,87.4,87.9,92.0,94.5,113.9$, $116.8,122.2,123.6,128.4,128.6,129.0,129.1,131.3,131.7,135.0,135.5,153.6$. HRMS (FAB) calcd for $\mathrm{C}_{70} \mathrm{H}_{58} \mathrm{O}_{6}\left(\mathrm{M}^{+}\right)$: 994.4233. Found: 994.4216 .

\section{1,4-Dibromo-2,5-dibutyl-3,6-diiodobenzene 11}

The title compound was synthesized according to the synthetic procedure of the related<smiles>Brc1c(Br)c(Br)c(Br)c(Br)c1Br</smiles>
compound, 2,5-dibromo-3,6-diiodo- $p$-xylene. ${ }^{4}$ To a solution of 1,4-dibromo-2,5dibutylbenzene $^{5}(2.1 \mathrm{~g}, 6.0 \mathrm{mmol}), \mathrm{K}_{2} \mathrm{~S}_{2} \mathrm{O}_{8}(2.7 \mathrm{~g}, 21 \mathrm{mmol})$, and $\mathrm{I}_{2}(5.8 \mathrm{~g}, 23 \mathrm{mmol})$ in $\mathrm{CH}_{2} \mathrm{Cl}_{2}(60 \mathrm{~mL})$ was slowly added $\mathrm{H}_{2} \mathrm{SO}_{4}(1.2 \mathrm{~mL})$ in TFA $(20 \mathrm{~mL})$ at $0{ }^{\circ} \mathrm{C}$. After stirring in the dark at $60{ }^{\circ} \mathrm{C}$ for 2 days, an aliquot was analyzed by ${ }^{1} \mathrm{H}$ NMR. If the reaction was not completed, to this mixture were added $\mathrm{K}_{2} \mathrm{~S}_{2} \mathrm{O}_{8}(2.7 \mathrm{~g}, 21 \mathrm{mmol})$ and $\mathrm{I}_{2}(5.8 \mathrm{~g}, 23 \mathrm{mmol})$, successively, and stirred at $60{ }^{\circ} \mathrm{C}$ for one more day. The reaction mixture was filtered and the solid was washed with $\mathrm{CH}_{2} \mathrm{Cl}_{2}(15 \mathrm{~mL} \times 2)$. Because the title compound is easy to be dissolved in $\mathrm{CH}_{2} \mathrm{Cl}_{2}$, it is not necessary to thoroughly wash the solid. The filtrate was washed with ice-cold $\mathrm{H}_{2} \mathrm{O}(100 \mathrm{~mL})$, sat. sodium sulfite aqueous solution $(100 \mathrm{~mL})$, and brine $(100 \mathrm{~mL})$. The combined organic solution was dried over $\mathrm{MgSO}_{4}$ and filtered on silica gel pad $(\sim 5 \mathrm{~cm})$. The silica gel pad was washed with $\mathrm{CH}_{2} \mathrm{Cl}_{2}$ $(50 \mathrm{~mL})$. Organic solvents were removed under reduced pressure and the pale brown residue was washed with cold hexane to afford $11(3.2 \mathrm{~g}, 5.3 \mathrm{mmol}, 89 \%)$ as a pale brown solid. $\mathrm{mp} \mathrm{105.0-}$ $110.2^{\circ} \mathrm{C}$ : IR (ATR) 2952, 2922, 2868, 2856, 1464, 1453, 1334, 1273, 1193, 1104, 1072, 998, 924, 901, 804, $725 \mathrm{~cm}^{-1}$; ${ }^{1} \mathrm{H}$ NMR (400 MHz, $\left.\mathrm{CDCl}_{3}, 25^{\circ} \mathrm{C}\right) \delta 0.98(\mathrm{t}, J=7.1 \mathrm{~Hz}, 6 \mathrm{H}), 1.44-1.55(\mathrm{~m}, 8 \mathrm{H})$, 3.32-3.38 (m, 4H). ${ }^{13} \mathrm{C}$ NMR (100 MHz, $\left.\mathrm{CDCl}_{3}, 25{ }^{\circ} \mathrm{C}\right): \delta 13.8,22.7,29.7,49.0,110.8,130.9,146.2$. HRMS (FAB) calcd for $\mathrm{C}_{14} \mathrm{H}_{18} \mathrm{Br}_{2} \mathrm{I}_{2}\left(\mathrm{M}^{+}\right)$: 597.7861. Found 597.7865.

\section{Compound 12}

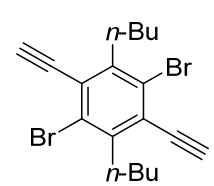

A solution of 11 (3.2 g, $5.3 \mathrm{mmol})$ and TMSA (1.8 mL, $13 \mathrm{mmol}, 2.4$ equiv) in toluene $(20 \mathrm{~mL})$ and diisopropylamine $(8 \mathrm{~mL})$ was deoxygenized by freeze-pumpthaw cycling. To the solution were added $\mathrm{CuI}(0.10 \mathrm{~g}, 0.53 \mathrm{mmol})$ and $\mathrm{Pd}\left(\mathrm{PPh}_{3}\right)_{4}$ $(0.24 \mathrm{~g}, 0.21 \mathrm{mmol})$ at room temperature. After stirring at $60^{\circ} \mathrm{C}$ overnight, an aliquot was analyzed by ${ }^{1} \mathrm{H}$ NMR. The methylene protons at benzylic positions were observed at $3.01 \mathrm{ppm}$. Those of mono, tri, and tetra-substituted compounds were observed at 3.10-3.20, 2.97, and 2.95 ppm, respectively. If the reaction was not completed, to this mixture were added $\mathrm{CuI}$ and $\mathrm{Pd}\left(\mathrm{PPh}_{3}\right)_{4}$, and 


\section{WILEY-VCH}

stirred at $60{ }^{\circ} \mathrm{C}$ for several hours. The resulting mixture was diluted with $\mathrm{CHCl}_{3}(100 \mathrm{~mL})$ and washed with sat. $\mathrm{NH}_{4} \mathrm{Cl}$ aqueous solution $(30 \mathrm{~mL})$. The aqueous layer was extracted with $\mathrm{CHCl}_{3}(15$ $\mathrm{mL} \times 2$ ) and the combined organic solution was dried over $\mathrm{MgSO}_{4}$. Organic solvents were removed under reduced pressure and the brown residue was filtered using short silica gel pad with hexane/AcOEt (v:v = 10:1) as an eluent. Organic solvents were removed under reduced pressure and the residue was subjected to GPC purification (eluent: $\mathrm{CHCl}_{3}$ ) to afford the coupling product $(1.7 \mathrm{~g}$, $3.1 \mathrm{mmol}, 59 \%$ ) as a brown solid. ${ }^{1} \mathrm{H}$ NMR (400 MHz, $\left.\mathrm{CDCl}_{3}, 25{ }^{\circ} \mathrm{C}\right) \delta 0.27$ (s, 18H), $0.96(\mathrm{t}, J=7.3$ $\mathrm{Hz}, 6 \mathrm{H}), 1.38-1.47(\mathrm{~m}, 4 \mathrm{H}), 1.48-1.58(\mathrm{~m}, 4 \mathrm{H}), 2.99-3.03(\mathrm{~m}, 4 \mathrm{H}) .{ }^{13} \mathrm{C} \mathrm{NMR}\left(100 \mathrm{MHz}, \mathrm{CDCl}_{3}\right.$, $\left.25^{\circ} \mathrm{C}\right): \delta-0.2,13.9,23.0,30.6,36.5,102.3,105.2,126.2,126.7,144.2$.

To a solution of the coupling product $(0.31 \mathrm{~g}, 0.57 \mathrm{mmol})$ in THF $(5 \mathrm{~mL})$ and $\mathrm{MeOH}(5 \mathrm{~mL})$ was added potassium carbonate $(0.28 \mathrm{~g}, 2.0 \mathrm{mmol})$ at room temperature. After stirring at room temperature for $0.5 \mathrm{~h}$, the resulting mixture was diluted with EtOAc $(30 \mathrm{~mL})$ and washed with water $(50 \mathrm{~mL})$ and brine $(20 \mathrm{~mL})$. The combined organic solution was dried over $\mathrm{MgSO}_{4}$. Organic solvents were removed under reduced pressure. The brown residue was subjected to short column chromatography on $\mathrm{SiO}_{2}$ with hexane as an eluent to afford $12(0.20 \mathrm{~g}, 0.50 \mathrm{mmol}, 89 \%, \mathrm{rf}=0.53$ (hexane $100 \%$, starting material: $\mathrm{rf}=0.60$ ) ) as a pale yellow solid. $\mathrm{mp} 75.2-76.7^{\circ} \mathrm{C}$; IR (ATR) 3290 , 2958, 2930, 2862, 2104 (C $\equiv C), 1480,1463,1434,1186,1136,1092,1072,998,923,842,741,693$ $\mathrm{cm}^{-1} ;{ }^{1} \mathrm{H}$ NMR $\left(300 \mathrm{MHz}, \mathrm{CDCl}_{3}, 25^{\circ} \mathrm{C}\right) \delta 0.96(\mathrm{t}, J=7.1 \mathrm{~Hz}, 6 \mathrm{H}), 1.38-1.62(\mathrm{~m}, 8 \mathrm{H}), 3.01-3.07$ (m, $4 \mathrm{H}), 3.65$ (s, 2H). ${ }^{13} \mathrm{C}$ NMR $\left(75 \mathrm{MHz}, \mathrm{CDCl}_{3}, 25{ }^{\circ} \mathrm{C}\right): \delta 13.8,22.9,30.7,36.4,81.1,86.9,125.8$, 127.1, 144.7. HRMS (FAB) calcd for $\mathrm{C}_{18} \mathrm{H}_{20} \mathrm{Br}_{2}\left(\mathrm{M}^{+}\right)$: 393.9932. Found 393.9946.

\section{Compound 13}

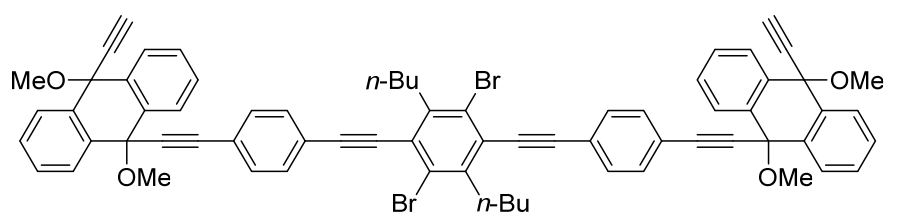

A solution of $12(0.14 \mathrm{~g}, 0.35 \mathrm{mmol})$ and $6(0.46 \mathrm{~g}, 0.76 \mathrm{mmol})$ in $\mathrm{Et}_{3} \mathrm{~N}(2 \mathrm{~mL})$ and THF ( $2 \mathrm{~mL})$ was deoxygenized by freezepump-thaw cycling. To the solution were added $\mathrm{CuI}(6.7 \mathrm{mg}, 35 \mu \mathrm{mol})$ and $\mathrm{Pd}\left(\mathrm{PPh}_{3}\right)_{4}(16 \mathrm{mg}, 14 \mu \mathrm{mol})$ at room temperature. After stirring at $60{ }^{\circ} \mathrm{C}$ for $3 \mathrm{~h}$, the resulting mixture was diluted with EtOAc $(10 \mathrm{~mL})$ and washed with sat. $\mathrm{NH}_{4} \mathrm{Cl}$ aqueous solution $(10 \mathrm{~mL})$. The organic solution was dried over $\mathrm{MgSO}_{4}$. Organic solvents were removed under reduced pressure and the yellow residue was subjected to column chromatography on $\mathrm{SiO}_{2}$ with hexane/EtOAc (v:v $\left.=10: 1\right)$ as an eluent to afford hexayne having TBS groups $(0.48 \mathrm{~g}, 0.35$ mmol, $\sim 99 \%, r f=0.20($ hexane/EtOAc $=10: 1))$ as a yellow solid. ${ }^{1} \mathrm{H} \mathrm{NMR}\left(400 \mathrm{MHz}, \mathrm{CDCl}_{3}, 25{ }^{\circ} \mathrm{C}\right)$ $\delta 0.14(\mathrm{~s}, 12 \mathrm{H}), 0.92(\mathrm{~s}, 18 \mathrm{H}), 0.97(\mathrm{t}, J=7.3 \mathrm{~Hz}, 6 \mathrm{H}), 1.46-1.49(\mathrm{~m}, 4 \mathrm{H}), 1.60-1.68(\mathrm{~m}, 4 \mathrm{H}), 3.05(\mathrm{~s}$, $6 \mathrm{H}), 3.06(\mathrm{~s}, 6 \mathrm{H}), 3.11(\mathrm{t}, J=8.3 \mathrm{~Hz}, 4 \mathrm{H}), 7.44-7.58(\mathrm{~m}, 16 \mathrm{H}), 8.02-8.08(\mathrm{~m}, 8 \mathrm{H})$.

To a solution of hexayne $(0.47 \mathrm{~g}, 0.35 \mathrm{mmol})$ in THF $(5 \mathrm{~mL})$ was added tetra- $n$-butylammonium fluoride ( $1 \mathrm{M}$ in THF, $0.80 \mathrm{~mL}, 0.80 \mathrm{mmol})$ at $0{ }^{\circ} \mathrm{C}$. After stirring at $\mathrm{rt}$ for $30 \mathrm{~min}$, organic solvents were removed under reduced pressure. The residue was diluted with $\mathrm{CH}_{2} \mathrm{Cl}_{2}(10 \mathrm{~mL})$ and washed with brine $(10 \mathrm{~mL})$. The aqueous layer was extracted with $\mathrm{CH}_{2} \mathrm{Cl}_{2}(10 \mathrm{~mL})$ and the combined organic 


\section{WILEY-VCH}

solution was dried over $\mathrm{MgSO}_{4}$. Organic solvents were removed under reduced pressure. The brown residue was subjected to column chromatography on $\mathrm{SiO}_{2}$ with hexane/EtOAc (v:v $\left.=8: 1\right)$ as an eluent to afford hexayne $13(0.39 \mathrm{~g}, 0.35 \mathrm{mmol}, \sim 99 \%, \mathrm{rf}=0.30$ (hexane/EtOAc $=4: 1))$ as a yellow solid. mp 94.5-95.9 ${ }^{\circ} \mathrm{C}$; IR (ATR) 3291, 2955, 2930, 2871, 2820, $2220(\mathrm{C} \equiv \mathrm{C}), 2113(\mathrm{C} \equiv \mathrm{C}), 1509,1449$, $1242,1072,1022,909,835,759,714 \mathrm{~cm}^{-1} ;{ }^{1} \mathrm{H} \mathrm{NMR}\left(400 \mathrm{MHz}, \mathrm{CDCl}_{3}, 25^{\circ} \mathrm{C}\right) \delta 0.97(\mathrm{t}, J=7.3 \mathrm{~Hz}$, $6 \mathrm{H}), 1.45-1.53(\mathrm{~m}, 4 \mathrm{H}), 1.60-1.68(\mathrm{~m}, 4 \mathrm{H}), 2.80(\mathrm{~s}, 2 \mathrm{H}), 3.00(\mathrm{~s}, 6 \mathrm{H}), 3.03(\mathrm{~s}, 6 \mathrm{H}), 3.10(\mathrm{t}, J=8.3 \mathrm{~Hz}$, 4H), 7.43-7.49 (m, 8H), 7.50-7.55 (m, 8H), 8.01-8.08 (m, 8H). $\left.{ }^{13} \mathrm{C} \mathrm{NMR} \mathrm{(100} \mathrm{MHz,} \mathrm{CDCl}_{3}, 25{ }^{\circ} \mathrm{C}\right): \delta$ $13.9,23.0,30.9,36.6,51.5,51.6,72.5,73.1,75.7,84.7,87.1,89.7,92.5,98.4,122.9,122.9,126.1$, $126.9,128.4,128.6,129.1,129.2,131.3,131.8,135.0,135.4,143.8$. HRMS (FAB) calcd for $\mathrm{C}_{70} \mathrm{H}_{56} \mathrm{O}_{4} \mathrm{Br}_{2}\left(\mathrm{M}^{+}\right):$1118.2545. Found 1118.2546.

\subsection{Synthesis of CPPA precursors 14}

[8]-, [9]-, and [10]CPPA precursors 14 were synthesized from diiodide 4 and the corresponding alkynes 7, 8, 10, and 13 under $\mathrm{Pd}$ and $\mathrm{Cu}$ catalysis.
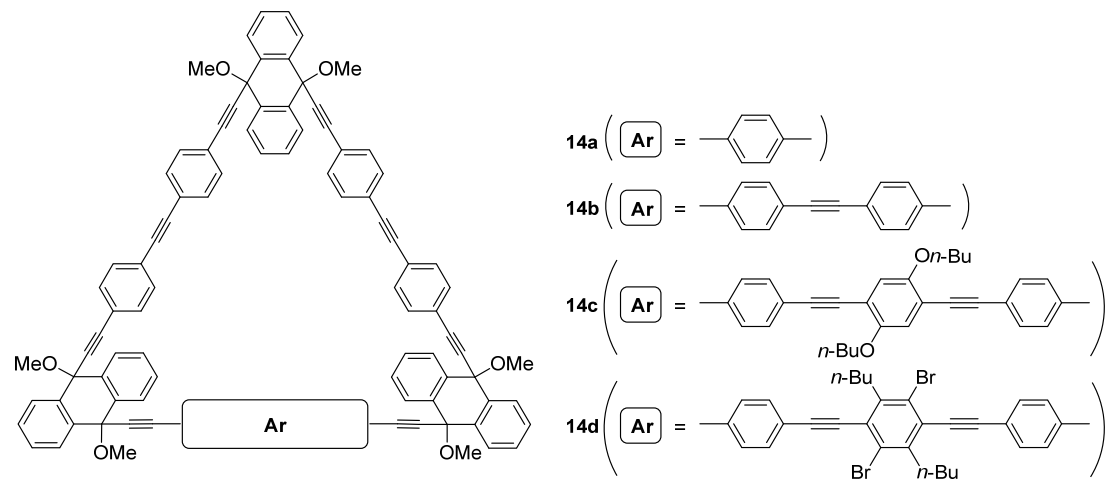

Typical synthetic procedure is as follows; a solution of 4 (70 mg, $79 \mu \mathrm{mol})$ and 7 (52 $\mathrm{mg}, 79 \mu \mathrm{mol})$ in $\mathrm{Et}_{3} \mathrm{~N}(3.0 \mathrm{~mL})$ and THF $(12 \mathrm{~mL})$ was deoxygenized by freeze-pump-thaw cycling. To the solution were added $\mathrm{CuI}(6.0 \mathrm{mg}, 32 \mu \mathrm{mol})$ and $\mathrm{Pd}\left(\mathrm{PPh}_{3}\right)_{4}(28 \mathrm{mg}, 24 \mu \mathrm{mol})$ at room temperature. After stirring at $60{ }^{\circ} \mathrm{C}$ for $20 \mathrm{~h}$, the resulting mixture was diluted with $\mathrm{CHCl}_{3}(20 \mathrm{~mL})$ and the organic solution was washed with sat. $\mathrm{NH}_{4} \mathrm{Cl}$ aqueous solution $(10 \mathrm{~mL})$. The aqueous layer was extracted with $\mathrm{CHCl}_{3}(20 \mathrm{~mL} \times 3)$ and the combined organic solution was dried over $\mathrm{MgSO}_{4}$. Organic solvents were passed through short Florisil column and removed under reduced pressure and the reddish brown residue was purified by GPC to afford 14a (36 mg, $28 \mu \mathrm{mol}, 35 \%$ ) as a brown solid. $\mathrm{mp} 193.7{ }^{\circ} \mathrm{C}$

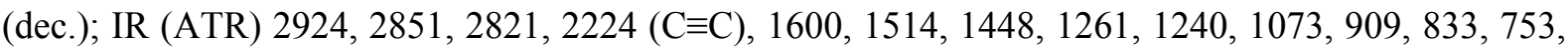
$729 \mathrm{~cm}^{-1} ;{ }^{1} \mathrm{H}$ NMR $\left(400 \mathrm{MHz}, \mathrm{CDCl}_{3}, 25^{\circ} \mathrm{C}\right) \delta 2.79(\mathrm{~s}, 6 \mathrm{H}), 2.79(\mathrm{~s}, 6 \mathrm{H}), 2.94(\mathrm{~s}, 6 \mathrm{H}), 7.14(\mathrm{~d}, J=8.5$ $\mathrm{Hz}, 4 \mathrm{H}), 7.22$ (d, $J=8.5 \mathrm{~Hz}, 4 \mathrm{H}), 7.25-7.26(\mathrm{~m}, 4 \mathrm{H}), 7.28-7.30(\mathrm{~m}, 8 \mathrm{H}), 7.50-7.53(\mathrm{~m}, 12 \mathrm{H}), 7.91-$ $7.96(\mathrm{~m}, 12 \mathrm{H}) .{ }^{13} \mathrm{C} \mathrm{NMR}\left(100 \mathrm{MHz}, \mathrm{CDCl}_{3}, 25{ }^{\circ} \mathrm{C}\right): \delta 50.8,51.2,71.3,71.4,85.2,85.2,86.1,90.7$, 90.7, 93.2, 94.1, 94.2, 122.4, 122.4, 122.5, 122.8, 122.9, 127.2, 128.4, 129.3, 129.5, 131.1, 131.1, 131.5, 131.6, 131.8, 134.8, 135.1. HRMS (FAB) calcd for $\mathrm{C}_{93} \mathrm{H}_{59} \mathrm{O}_{5}\left([\mathrm{M}-\mathrm{OMe}]^{+}\right)$: 1255.4357. Found 1255.4380 . 


\section{WILEY-VCH}

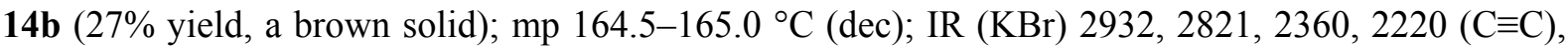
$1514,1448,1278,1239,1078,957,910,836,755 \mathrm{~cm}^{-1}$; ${ }^{1} \mathrm{H}$ NMR $\left(400 \mathrm{MHz}, \mathrm{CDCl}_{3}, 25^{\circ} \mathrm{C}\right) \delta 2.81(\mathrm{~s}$, $18 \mathrm{H}), 7.30(\mathrm{~d}, J=8.8 \mathrm{~Hz}, 12 \mathrm{H}), 7.33(\mathrm{~d}, J=8.8 \mathrm{~Hz}, 12 \mathrm{H}), 7.53(\mathrm{dd}, J=3.4,5.9 \mathrm{~Hz}, 12 \mathrm{H}), 7.96(\mathrm{dd}, J$ $=3.4,5.9 \mathrm{~Hz}, 12 \mathrm{H}) ;{ }^{13} \mathrm{C} \mathrm{NMR}\left(75 \mathrm{MHz}, \mathrm{CDCl}_{3}, 25{ }^{\circ} \mathrm{C}\right) \delta 50.7,71.2,85.3,90.7,94.0,122.4,122.9$, 128.1, 129.4, 131.1, 131.7, 134.9. HRMS (FAB) calcd for $\mathrm{C}_{101} \mathrm{H}_{63} \mathrm{O}_{5}\left([\mathrm{M}-\mathrm{OMe}]^{+}\right)$: 1355.4676. Found 1355.4658 .

14c $(27 \%$ yield, a brown solid containing a small amount of inseparable byproducts, which could be removed after the next reductive aromatization reaction); ${ }^{1} \mathrm{H} \mathrm{NMR}\left(500 \mathrm{MHz}, \mathrm{CDCl}_{3}, 25^{\circ} \mathrm{C}\right): \delta 0.89$ (t, $J=7.6 \mathrm{~Hz}, 6 \mathrm{H}), 1.42-1.51(\mathrm{~m}, 4 \mathrm{H}), 1.71-1.76(\mathrm{~m}, 4 \mathrm{H}), 2.90(\mathrm{~s}, 6 \mathrm{H}), 2.90(\mathrm{~s}, 6 \mathrm{H}), 2.91(\mathrm{~s}, 6 \mathrm{H}), 3.93(\mathrm{t}$, $J=6.4 \mathrm{~Hz}, 4 \mathrm{H}), 6.90(\mathrm{~s}, 2 \mathrm{H}), 7.21-7.24(\mathrm{~m}, 8 \mathrm{H}), 7.28-7.30(\mathrm{~m}, 8 \mathrm{H}), 7.38$ (br s, 8H), 7.51-7.55 (m, $12 \mathrm{H})$, 7.93-7.95 (m, 8H), 8.00-8.01 (m, 4H). HRMS (FAB) calcd for $\mathrm{C}_{117} \mathrm{H}_{83} \mathrm{O}_{7}\left([\mathrm{M}-\mathrm{OMe}]^{+}\right)$: 1600.6173. Found 1600.6168.

In the case of 14d, to avoid the debromination under Pd-catalyzed coupling conditions, we carried out the coupling reaction of 4 and 13 using smaller amounts of $\mathrm{Pd}(5 \mathrm{~mol} \%)$ and $\mathrm{Cu}(10 \mathrm{~mol} \%)$

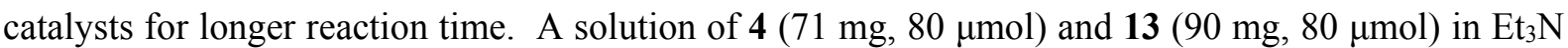
$(3.0 \mathrm{~mL})$ and THF $(12 \mathrm{~mL})$ was deoxygenized by freeze-pump-thaw cycling. To the solution were added $\mathrm{CuI}(3.0 \mathrm{mg}, 0.016 \mathrm{mmol})$ and $\mathrm{Pd}\left(\mathrm{PPh}_{3}\right)_{4}(9.2 \mathrm{mg}, 8.0 \mu \mathrm{mol})$ at room temperature. After stirring at $60{ }^{\circ} \mathrm{C}$ for $3 \mathrm{~d}$, the resulting mixture was diluted with $\mathrm{CHCl}_{3}(20 \mathrm{~mL})$ and the organic solution was washed with sat. $\mathrm{NH}_{4} \mathrm{Cl}$ aqueous solution $(10 \mathrm{~mL})$. The following purification procedure was same as described above.

14d $(27 \mathrm{mg}, 19 \%$ yield, a yellow solid containing a small amount of inseparable byproducts, which could be removed after the next reductive aromatization reaction); ${ }^{1} \mathrm{H} \mathrm{NMR}\left(400 \mathrm{MHz}, \mathrm{CDCl}_{3}, 25{ }^{\circ} \mathrm{C}\right)$ $\delta 0.90(\mathrm{t}, J=7.1 \mathrm{~Hz}, 6 \mathrm{H}), 1.37-1.45(\mathrm{~m}, 4 \mathrm{H}), 1.53-1.65(\mathrm{~m}, 4 \mathrm{H}), 2.89(\mathrm{~s}, 12 \mathrm{H}), 2.92(\mathrm{~s}, 6 \mathrm{H}), 3.00-3.08$ $(\mathrm{m}, 4 \mathrm{H})$, 7.24-7.39 (m, 24H), 7.51-7.54 (m, 12H), 7.94-7.97 (m, 8H), 7.99-8.01 (m, 4H). HRMS (FAB) calcd for $\mathrm{C}_{117} \mathrm{H}_{81} \mathrm{O}_{5} \mathrm{Br}_{2}\left([\mathrm{M}-\mathrm{OMe}]^{+}\right)$: 1723.4451. Found 1723.4440.

\subsection{Synthesis of CPPAs 1}

[8]-, [9]-, and [10]CPPAs were obtained from their precursors 14 through tin-mediated reductive aromatization. The tin-mediated reductive aromatization procedure reported by Yamago research group $^{6}$ was applied to synthesize CPPAs 1. Degassed THF (1 mL), $\mathrm{SnCl}_{2} \cdot 2 \mathrm{H}_{2} \mathrm{O}(4.6 \mathrm{mg}, 20 \mu \mathrm{mol})$, and $3 \mathrm{M} \mathrm{HCl}$ aqueous solution (degassed, $14 \mu \mathrm{L}, 42 \mu \mathrm{mol}$ ) were successively added to $25 \mathrm{~mL}$ Schlenk tube and well-mixed at room temperature for $15 \mathrm{~min}$. To the mixture was added the precursor of $\mathbf{1 d}$ $(10 \mathrm{mg}, 5.7 \mu \mathrm{mol})$ in degassed THF $(4 \mathrm{~mL})$ at room temperature. After stirring at room temperature in the dark for $6 \mathrm{~h}$, the resulting mixture was diluted with THF $(10 \mathrm{~mL})$ and the organic solution was washed with sat. $\mathrm{NaHCO}_{3}(20 \mathrm{~mL})$. The aqueous layer was extracted with THF $(20 \mathrm{~mL} \times 2)$ and the combined organic solution was dried over $\mathrm{MgSO}_{4}$. Organic solvents were removed under reduced pressure. The yellowish brown residue was purified by GPC (eluent: $\left.\mathrm{CHCl}_{3}\right)$ to afford $\mathbf{1 d}(4.0 \mathrm{mg}, 2.5$ $\mu \mathrm{mol}, 45 \%)$ as an orange solid. 

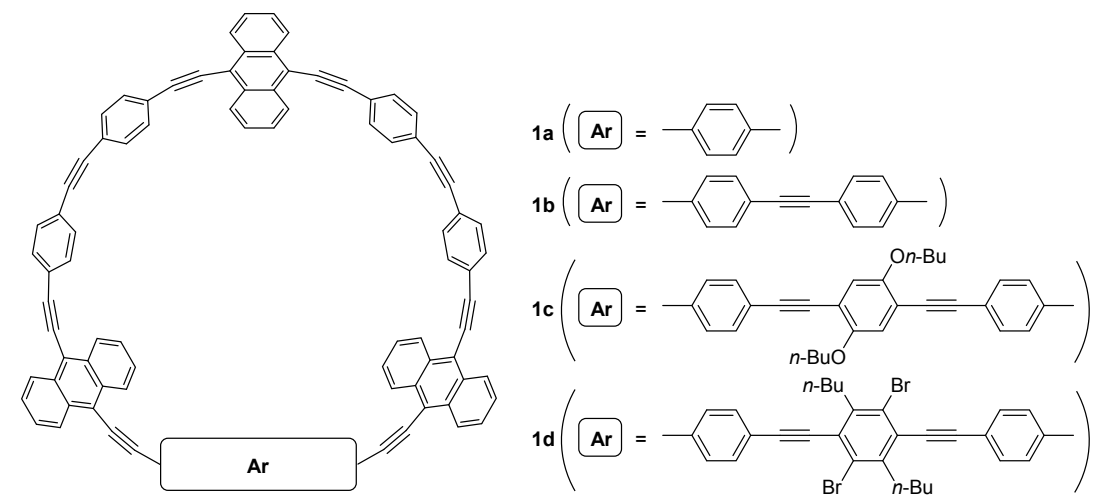

1a (87\% yield (determined by ${ }^{1} \mathrm{H}$ NMR using 1,4-dioxane as a standard), a yellowish red solid); mp: not measured due to gradual decomposition in solid state; IR (ATR) 2997, 2923, 2922, 2168 (C $\equiv \mathrm{C}$ ), $1729,1588,1511,1436,1400,1100,1027,834,802 \mathrm{~cm}^{-1}$; ${ }^{1} \mathrm{H}$ NMR (400 MHz, $\left.\mathrm{CDCl}_{3}, 25^{\circ} \mathrm{C}\right): \delta 7.43$ $(\mathrm{d}, J=8.0 \mathrm{~Hz}, 8 \mathrm{H}), 7.55-7.63(\mathrm{~m}, 24 \mathrm{H}), 8.55-8.58(\mathrm{~m}, 12 \mathrm{H}) ;{ }^{13} \mathrm{C} \mathrm{NMR}\left(100 \mathrm{MHz}, \mathrm{CDCl}_{3}, 25{ }^{\circ} \mathrm{C}\right) \delta$ 93.4, 93.5, 93.6, 95.0, 104.5, 104.6, 104.6, 119.0, 119.0, 119.1, 123.6, 124.0, 124.0, 126.9, 127.0, 131.2, 131.3, 131.4, 132.1. HRMS (FAB) calcd for $\mathrm{C}_{88} \mathrm{H}_{44}\left(\mathrm{M}^{+}\right)$: 1100.3443. Found: 1100.3434 .

1b (27\% yield, a yellowish red solid); decomposition temp.: 180-200 ${ }^{\circ} \mathrm{C}$; IR (KBr) 2922, 2851, 2166 $(\mathrm{C} \equiv \mathrm{C}), 1637,1509,1261,1100,1026,834,764,637 \mathrm{~cm}^{-1} ;{ }^{1} \mathrm{H}$ NMR $\left(400 \mathrm{MHz}, \mathrm{CDCl}_{3}, 25{ }^{\circ} \mathrm{C}\right) \delta 7.47$ $(\mathrm{d}, J=8.4 \mathrm{~Hz}, 12 \mathrm{H}), 7.58-7.62(\mathrm{~m}, 24 \mathrm{H}), 8.58(\mathrm{dd}, J=3.3,6.6 \mathrm{~Hz}, 12 \mathrm{H}) ;{ }^{13} \mathrm{C} \mathrm{NMR}\left(100 \mathrm{MHz}, \mathrm{CDCl}_{3}\right.$, $\left.25^{\circ} \mathrm{C}\right) \delta 92.5,94.3,104.1,118.9,123.6,123.9,126.9,127.0,131.3,131.5,132.1$. HRMS (FAB) calcd for $\mathrm{C}_{96} \mathrm{H}_{49}\left(\mathrm{M}+\mathrm{H}^{+}\right)$: 1201.3834. Found 1201.3839.

1c (40\% yield, a yellowish red solid); decomposition temp.: 190-210 ${ }^{\circ} \mathrm{C}$; IR (ATR) 2923, 2853, 2189 $(\mathrm{C} \equiv \mathrm{C}), 1671,1596,1512,1492,1402,1213,1100,1026,832 \mathrm{~cm}^{-1} ;{ }^{1} \mathrm{HNMR}\left(500 \mathrm{MHz}, \mathrm{CDCl}_{3}\right.$, $\left.25^{\circ} \mathrm{C}\right): \delta 0.98(\mathrm{t}, J=7.3 \mathrm{~Hz}, 6 \mathrm{H}), 1.52-1.59(\mathrm{~m}, 4 \mathrm{H}), 1.77-1.85(\mathrm{~m}, 4 \mathrm{H}), 4.00(\mathrm{t}, J=6.4 \mathrm{~Hz}, 4 \mathrm{H}), 6.92$ (s, 2H), 7.48-7.52 (m, 12H), 7.60-7.65 (m, 24H), 8.59-8.61 (m, 12H); ${ }^{13} \mathrm{C} \mathrm{NMR} \mathrm{(125} \mathrm{MHz,} \mathrm{CDCl}_{3}$, $\left.25^{\circ} \mathrm{C}\right): \delta 13.9,19.2,31.3,69.2,91.2,91.7,91.8,91.9,93.8,96.2,103.8,104.0,114.3,116.5,118.8$, 118.8, 118.9, 123.4, 123.5, 123.8, 123.8, 124.0, 126.9, 127.1, 131.3, 131.4, 131.5, 131.5, 132.1, 132.1, 153.5. Several signals were overlapped in aromatic region. HRMS (FAB) calcd for $\mathrm{C}_{112} \mathrm{H}_{68} \mathrm{O}_{2}\left(\mathrm{M}^{+}\right)$: 1444.5219. Found: 1444.5209.

1d (45\% yield, an orange solid); decomposition temp.: 180-200 ${ }^{\circ} \mathrm{C}$; IR (ATR) 2958, 2920, 2851, 2180 $(\mathrm{C} \equiv \mathrm{C}), 2153(\mathrm{C} \equiv \mathrm{C}), 1659,1633,1596,1509,1463,1401,1260,1090,1052,1032,833,801,761,719$, 658, 637, 614, $570 \mathrm{~cm}^{-1}$; ${ }^{1} \mathrm{H}$ NMR (400 MHz, THF-d $\left.6,25^{\circ} \mathrm{C}\right): \delta 1.00(\mathrm{t}, J=7.3 \mathrm{~Hz}, 6 \mathrm{H}), 1.51-1.58$ (m, 4H), 1.60-1.67 (m, 4H), 3.12-3.16 (m, 4H), 7.59 (d, $J=8.2 \mathrm{~Hz}, 8 \mathrm{H}), 7.62(\mathrm{~d}, J=8.9 \mathrm{~Hz}, 4 \mathrm{H}), 7.67$ (dd, $J=3.2,6.4 \mathrm{~Hz}, 12 \mathrm{H}), 7.76(\mathrm{~d}, J=8.2 \mathrm{~Hz}, 8 \mathrm{H}), 7.79(\mathrm{~d}, J=8.9 \mathrm{~Hz}, 4 \mathrm{H}), 8.68(\mathrm{dd}, J=3.2,6.4 \mathrm{~Hz}$, $12 \mathrm{H}) ;{ }^{13} \mathrm{C}$ NMR (100 MHz, THF-d $\left.6,25{ }^{\circ} \mathrm{C}\right) \delta 13.4,22.9,29.8,36.4,91.4,91.4,91.7,92.5,93.7,99.9$, 103.8, 103.9, 104.0, 118.6, 118.7, 118.8, 123.3, 123.8, 123.8, 124.4, 126.5, 126.6, 126.9, 127.1, 131.4, 131.5, 132.1, 132.1, 143.5. Several signals were overlapped in aromatic region. HRMS (FAB) calcd for $\mathrm{C}_{112} \mathrm{H}_{66} \mathrm{Br}_{2}\left(\mathrm{M}^{+}\right)$: 1571.3555. Found 1571.3551. 


\section{WILEY-VCH}

\subsection{UV-vis absorption spectra and photoluminescence spectra of 1}

Absorbance and photoluminescence spectra of 1 measured in $\mathrm{CHCl}_{3}$ were summarized in Figure $\mathrm{S} 1$. The molar extinction coefficients and quantum yields of $\mathbf{1}$ were summarized in Table S1. The absorbance and fluorescence maxima of $\mathbf{1 b}$, which were detected at $467 \mathrm{~nm}$ and $532 \mathrm{~nm}$, respectively, were quite similar to those of $\mathbf{1 c}$ and $\mathbf{1 d}$. On the other hand, both absorbance and fluorescence spectra of 1a were different from other CPPAs. From the TD-DFT calculation (see next section 3.2), the shoulder signal around $550 \mathrm{~nm}$ is assigned as vertical transitions including HOMO-LUMO transition, which is forbidden in $\mathbf{1 b}$, but allowed in 1a (Figsure S3 and S4). By considering that 1a emits much weaker fluorescence $(\Phi=0.005)$ than $\mathbf{1 b}-\mathbf{1 d}(\Phi=0.81-0.92)($ Table $\mathrm{S} 1)$, $\pi$-elongated system of $\mathbf{1 a}$ is different from other CPPAs.

a

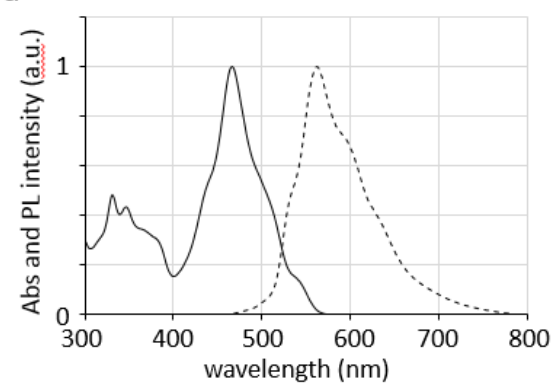

C

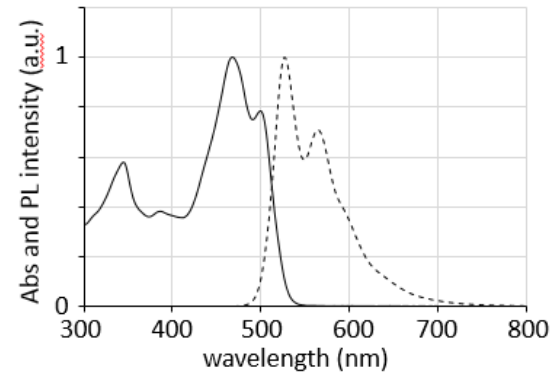

b

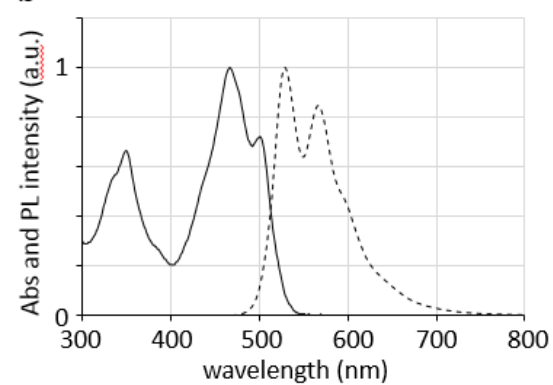

d

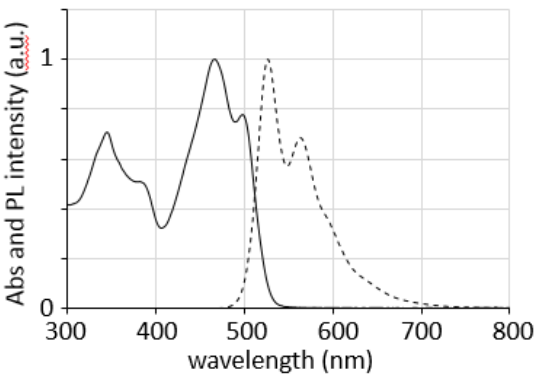

Figure S1. Absorption (solid) and photoluminescence (dashed) spectra of (a) 1a, (b) 1b, (c) 1c, and (d) 1 d.

Table S1. Absorption and phosphorescence properties of CPPA 1. ${ }^{a}$

\begin{tabular}{ccc}
\hline CPPA & $\begin{array}{c}\text { molar extinction coefficient } \\
\left(\varepsilon, \times 10^{4} \mathrm{~cm}^{-1} \mathrm{M}^{-1}\right)\end{array}$ & $\begin{array}{c}\text { relative quantum yield } \\
(\Phi, \%)\end{array}$ \\
\hline 1a & $5.9(467 \mathrm{~nm})$ & 0.5 \\
1b & $14.9(467 \mathrm{~nm}), 10.8(501 \mathrm{~nm})$ & 90.0 \\
1c & $16.4(467 \mathrm{~nm}), 12.9(499 \mathrm{~nm})$ & 92.1 \\
1d & $14.0(468 \mathrm{~nm}), 11.1(500 \mathrm{~nm})$ & 81.2 \\
\hline
\end{tabular}

a Measured in $\mathrm{CHCl}_{3 .}$. Uranine was used as a standard. 


\subsection{DFT calculation of 1}

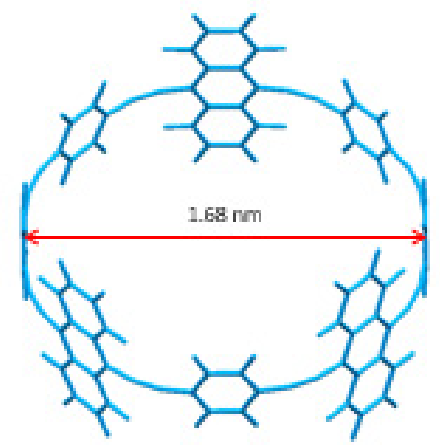

c

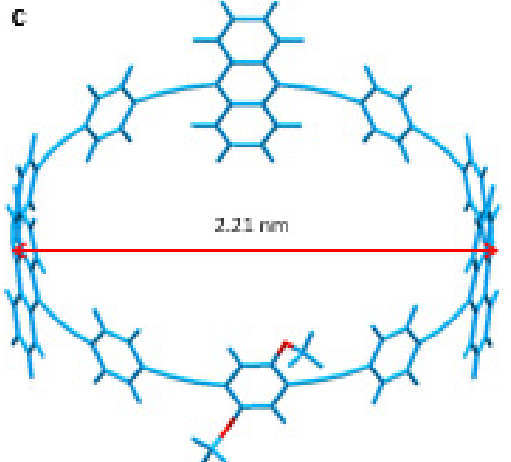

b

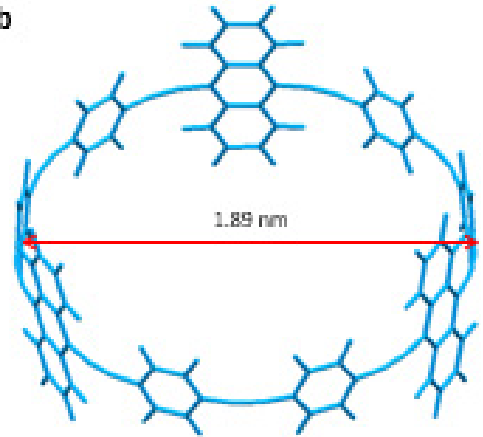

d

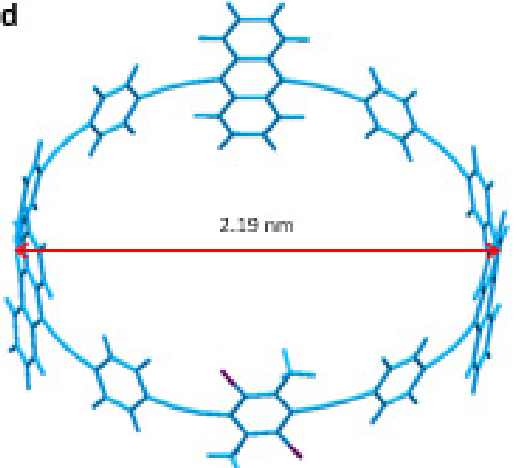

Figure S2. Optimized structures of CPPAs (a) 1a, (b) $\mathbf{1 b}$, (c) 1c, and (d) $\mathbf{1 d}$. 


\section{WILEY-VCH}

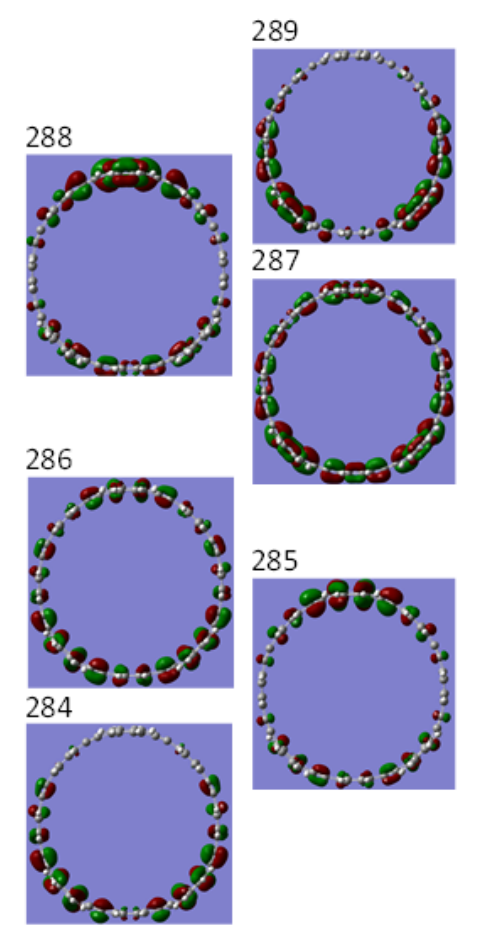

excited state: 1

energy gap: $2.22 \mathrm{eV}$

(559 nm)

oscillator strength: 0.09

LUMO+2 (289)

LUMO+1 (288)

LUMO (287)

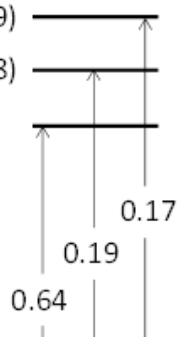

HOMO (286)

HOMO-1 (285)

HOMO-2 (284) excited state: 2

energy gap: $2.56 \mathrm{eV}$

$(485 \mathrm{~nm})$

oscillator strength: 2.03 oscillator strength: 1.63

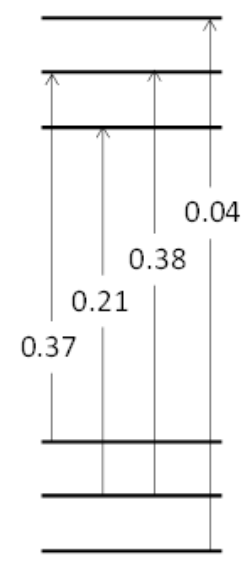

excited state: 3

energy gap: $2.67 \mathrm{eV}$

(464 nm)

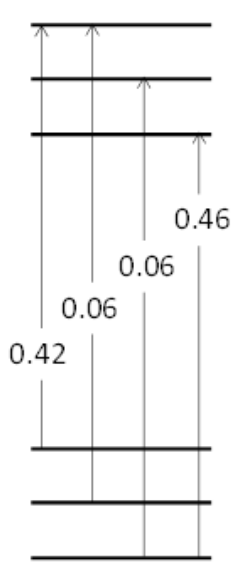

Figure S3. Summary of TD-DFT calculation of 1a.

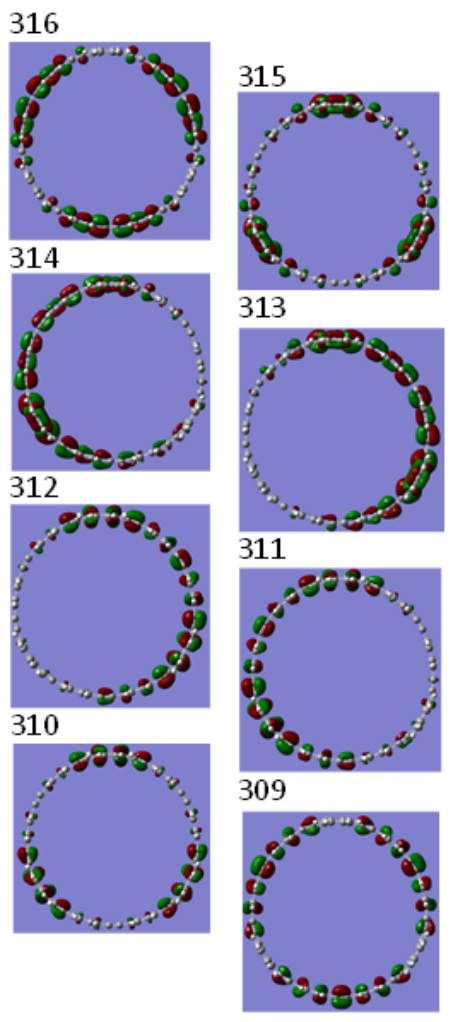

excited state: 1

energy gap: $2.30 \mathrm{eV}$ (539 nm)

oscillator strength: 0.00

LUMO+3 (316)

LUMO+2 (315)

LUMO+1 (314)

LUMO (313)

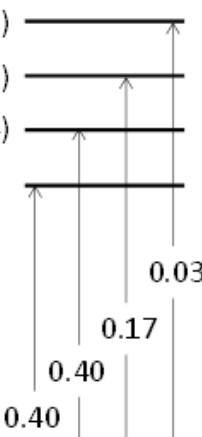

HOMO (312)

HOMO-1 (311)

HOMO-2 (310)

HOMO-3 (309) excited state: 2

energy gap: $2.59 \mathrm{eV}$

(479 nm)

oscillator strength: 2.15 oscillator strength: 2.19

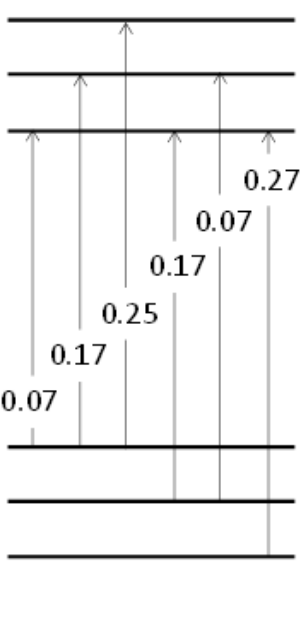

excited state: 3

energy gap: $2.59 \mathrm{eV}$

$(479 \mathrm{~mm})$

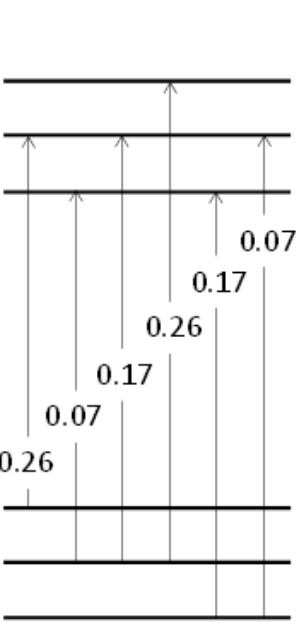

Figure S4. Summary of TD-DFT calculation of $\mathbf{1 b}$. 


\section{WILEY-VCH}

\subsection{Theoretical calculation of CNT@CPPA complexes}

To evaluate the complexation energy, we optimized the structures of CNT@CPPA, CNT, and CPPA by the programs, and estimated the total energy of them.

The energy difference $(\Delta E)$ before and after complexation was estimated by the following equation: “ $\Delta E=E(\mathrm{CNT} @ \mathrm{CPPA})-E(\mathrm{CNT})-E(\mathrm{CPPA}) "$, in which $E(\mathrm{CNT} @ \mathrm{CPPA}), E(\mathrm{CNT})$, and $E(\mathrm{CPPA})$ are total energies of CNT@CPPA, CNT, and CPPA.

Through the complexation, the structures of CPPA and CNT are deformed. By comparing total energies of CPPA and CNT before and after complexation, we evaluated the suitable combination of CNT and CPPA for complexation.

The energy difference $\left(\Delta E^{\prime}\right)$ between complexed CPPA and optimized CPPA (before complexation), which is a deformation index of CPPA, was estimated by the following equation: " $\Delta E$ ' $=E$ (CPPA in complex $)-E(\mathrm{CPPA}) "$. By removing CNT moieties from the optimized CNT@CPPA complex, the deformed structure of CPPA was obtained. The total energy of the deformed CPPA, E(CPPA in complex), was estimated through the single-point calculation.

The energy difference ( $\Delta E$ ") between complexed CNT and pristine CNT (before complexation), which is a deformation index of $\mathrm{CNT}$, was estimated by the following equation: " $\Delta E$ " $=E(\mathrm{CNT}$ in complex $)-E(\mathrm{CNT}) "$. By removing CPPA moieties from the optimized CNT@CPPA complex, the deformed structure of CNT was obtained. The total energy of the deformed CPPA, E(CPPA in complex), was estimated through the single-point calculation.

The estimated energy difference are summarized in Tables S2-S5 shown below.

Table S2. Free energy differences of CNT@1a.

\begin{tabular}{|c|c|c|c|c|c|c|c|}
\hline \multirow[b]{2}{*}{$\begin{array}{c}\text { chirality } \\
(n, m)\end{array}$} & \multirow{2}{*}{$\begin{array}{c}\text { diameter } \\
(\mathrm{nm})\end{array}$} & \multicolumn{3}{|c|}{ MMFF94 } & \multicolumn{3}{|c|}{ Forcite } \\
\hline & & $\begin{array}{c}\Delta E \\
(\mathrm{kcal} / \mathrm{mol})\end{array}$ & $\begin{array}{c}\Delta E^{\prime} \\
(\mathrm{kcal} / \mathrm{mol})\end{array}$ & $\begin{array}{c}\Delta E^{\prime \prime} \\
(\mathrm{kcal} / \mathrm{mol})\end{array}$ & $\underset{(\mathrm{kcal} / \mathrm{mol})}{\Delta E}$ & $\begin{array}{c}\Delta E^{\prime} \\
(\mathrm{kcal} / \mathrm{mol})\end{array}$ & $\begin{array}{c}\Delta E^{\prime \prime} \\
(\mathrm{kcal} / \mathrm{mol})\end{array}$ \\
\hline$(13,0)$ & 1.018 & -75.8 & 0.2 & 0.2 & -116.7 & 3.8 & 0.2 \\
\hline$(14,0)$ & 1.096 & -57.8 & 7.5 & 2.4 & -129.5 & 0.5 & 0.5 \\
\hline$(13,2)$ & 1.105 & - & 10.9 & 157.0 & -141.1 & 0.7 & 0.8 \\
\hline$(14,1)$ & 1.137 & -25.4 & 22.0 & 144.3 & -128.0 & 4.1 & 2.3 \\
\hline$(15,0)$ & 1.175 & 5.0 & 39.3 & 11.1 & -95.3 & 15.6 & 6.6 \\
\hline$(16,0)$ & 1.253 & 119.6 & 112.0 & 26.1 & 7.8 & 74.6 & 24.2 \\
\hline
\end{tabular}


WILEY-VCH

Table S3. Free energy differences of CNT@1b.

\begin{tabular}{|c|c|c|c|c|c|c|c|}
\hline \multirow{2}{*}{$\begin{array}{c}\text { chirality } \\
(n, m)\end{array}$} & \multirow{2}{*}{$\begin{array}{l}\text { diameter } \\
(\mathrm{nm})\end{array}$} & \multicolumn{3}{|c|}{ MMFF94 } & \multicolumn{3}{|c|}{ Forcite } \\
\hline & & $\begin{array}{c}\Delta E \\
(\mathrm{kcal} / \mathrm{mol})\end{array}$ & $\begin{array}{c}\Delta E^{\prime} \\
(\mathrm{kcal} / \mathrm{mol})\end{array}$ & $\begin{array}{c}\Delta E^{\prime \prime} \\
\text { (kcal/mol) }\end{array}$ & $\begin{array}{c}\Delta E \\
(\mathrm{kcal} / \mathrm{mol})\end{array}$ & $\begin{array}{c}\Delta E^{\prime} \\
\text { (kcal/mol) }\end{array}$ & $\begin{array}{c}\Delta E^{\prime \prime} \\
\text { (kcal/mol) }\end{array}$ \\
\hline$(15,0)$ & 1.175 & -66.8 & 4.8 & 0.3 & -111.8 & 6.7 & 0.5 \\
\hline$(16,0)$ & 1.253 & -83.3 & 1.5 & 0.1 & -137.8 & 3.1 & 0.2 \\
\hline$(16,1)$ & 1.294 & - & 0.6 & - & -154.5 & 1.0 & 0.3 \\
\hline$(15,3)$ & 1.308 & -89.8 & 2.6 & 1.3 & -156.2 & 0.8 & 0.3 \\
\hline$(17,0)$ & 1.331 & -84.6 & 1.3 & 0.8 & -144.1 & 1.1 & 1.3 \\
\hline$(18,0)$ & 1.409 & -54.4 & 17.5 & 4.6 & -99.0 & 23.5 & 10.4 \\
\hline
\end{tabular}

Table S4. Free energy differences of CNT@1c.

\begin{tabular}{|c|c|c|c|c|c|c|c|}
\hline \multirow{2}{*}{$\begin{array}{c}\text { chirality } \\
(n, m)\end{array}$} & \multirow{2}{*}{$\begin{array}{l}\text { diameter } \\
(\mathrm{nm})\end{array}$} & \multicolumn{3}{|c|}{ MMFF94 } & \multicolumn{3}{|c|}{ Forcite } \\
\hline & & $\begin{array}{c}\Delta E \\
(\mathrm{kcal} / \mathrm{mol})\end{array}$ & $\begin{array}{c}\Delta E^{\prime} \\
(\mathrm{kcal} / \mathrm{mol})\end{array}$ & $\begin{array}{c}\Delta E^{\prime \prime} \\
(\mathrm{kcal} / \mathrm{mol})\end{array}$ & $\begin{array}{c}\Delta E \\
(\mathrm{kcal} / \mathrm{mol})\end{array}$ & $\begin{array}{c}\Delta E^{\prime} \\
(\mathrm{kcal} / \mathrm{mol})\end{array}$ & $\begin{array}{c}\Delta E^{\prime \prime} \\
(\mathrm{kccal} / \mathrm{mol})\end{array}$ \\
\hline$(18,0)$ & 1.409 & -92.6 & 0.9 & 0.2 & -132.4 & 7.4 & 0.5 \\
\hline$(19,0)$ & 1.488 & -95.6 & 0.7 & 0.8 & -160.5 & 2.3 & 0.2 \\
\hline$(18,2)$ & 1.494 & - & 10.0 & - & -169.9 & 3.1 & 0.3 \\
\hline$(17,4)$ & 1.512 & -79.4 & 8.4 & 5.0 & -175.5 & 0.9 & 0.4 \\
\hline$(19,1)$ & 1.528 & -92.2 & 4.9 & 2.6 & -171.6 & 0.7 & 0.6 \\
\hline$(20,0)$ & 1.566 & -63.2 & 16.9 & 5.9 & -159.3 & 3.1 & 2.5 \\
\hline
\end{tabular}

Table S5. Free energy differences of CNT@1d.

\begin{tabular}{|c|c|c|c|c|c|c|c|}
\hline \multirow{2}{*}{$\begin{array}{l}\text { chirality } \\
(n, m)\end{array}$} & \multirow{2}{*}{$\begin{array}{l}\text { diameter } \\
(\mathrm{nm})\end{array}$} & \multicolumn{3}{|c|}{ MMFF94 } & \multicolumn{3}{|c|}{ Forcite } \\
\hline & & $\begin{array}{c}\Delta E \\
(\mathrm{kcal} / \mathrm{mol})\end{array}$ & $\begin{array}{c}\Delta E^{\prime} \\
(\mathrm{kcal} / \mathrm{mol})\end{array}$ & $\begin{array}{c}\Delta E^{\prime \prime} \\
(\mathrm{kcal} / \mathrm{mol})\end{array}$ & $\begin{array}{c}\Delta E \\
(\mathrm{kcal} / \mathrm{mol})\end{array}$ & $\begin{array}{c}\Delta E^{\prime} \\
(\mathrm{kcal} / \mathrm{mol})\end{array}$ & $\begin{array}{c}\Delta E^{\prime \prime} \\
(\mathrm{kcal} / \mathrm{mol})\end{array}$ \\
\hline$(17,0)$ & 1.331 & -79.6 & 4.9 & 0.2 & -114.4 & 11.6 & 0.3 \\
\hline$(18,0)$ & 1.409 & -95.3 & 1.3 & 0.2 & -138.6 & 7.4 & 0.3 \\
\hline$(16,4)$ & 1.435 & -108.2 & 17.2 & 0.5 & -153.2 & 7.0 & 0.5 \\
\hline$(17,3)$ & 1.463 & - & 0.6 & - & -162.1 & 5.4 & 0.4 \\
\hline$(19,0)$ & 1.488 & -99.5 & 1.7 & 0.8 & -51.2 & 2.2 & 0.3 \\
\hline$(20,0)$ & 1.566 & -66.6 & 18.9 & 5.9 & -162.7 & 3.7 & 2.4 \\
\hline
\end{tabular}

The diameters of CNTs were calculated by using the following equation: diameter $(\mathrm{nm})=(0.246 / \pi) \times$ $(n \times n+n \times m+m \times m)^{1 / 2}$, in which numbers $n$ and $m$ are chirality of CNTs. The theoretical calculation using MMFF94 and Forcite seems to overestimate the stability through $\pi-\pi$ interaction in CNT@CPPA complexes. We concluded that CNTs, whose $\Delta E^{\prime}$, and $\Delta E^{\prime \prime}$ values are nearby zero, is suitable for complexation with CPPAs. The diameters of CNTs, which can be fitted with CPPAs 1a-d, were estimated at $1.10 \mathrm{~nm}, 1.33 \mathrm{~nm}, 1.53 \mathrm{~nm}$, and $1.49 \mathrm{~nm}$, respectively (highlighted in Tables). 


\section{WILEY-VCH}

Energy-minimized structures of "tube-in-ring" and "ring-on-tube" complexes were also obtained by force field calculation by utilizing Forcite program (Table S6). Although "ring-on-tube" complexation is unstable compared with "tunbe-inring" complexation, this calculation results support that "ring-on-tube" complexation through $\pi-\pi$ and $\mathrm{C}-\mathrm{H}-\pi$ interaction is possible.

Table S6. Free energy differences of (14,0)-CNT@1a.

\begin{tabular}{cccc}
\hline $\begin{array}{c}\text { complexation } \\
\text { mode }\end{array}$ & $\begin{array}{c}\Delta E \\
(\mathrm{kcal} / \mathrm{mol})\end{array}$ & $\begin{array}{c}\Delta E^{\prime} \\
(\mathrm{kcal} / \mathrm{mol})\end{array}$ & $\begin{array}{c}\Delta E^{\prime \prime} \\
(\mathrm{kcal} / \mathrm{mol})\end{array}$ \\
\hline tube-in-ring & -142.1 & 0.6 & 0.5 \\
ring-on-tube & -57.8 & 15.1 & 0.5
\end{tabular}

a)

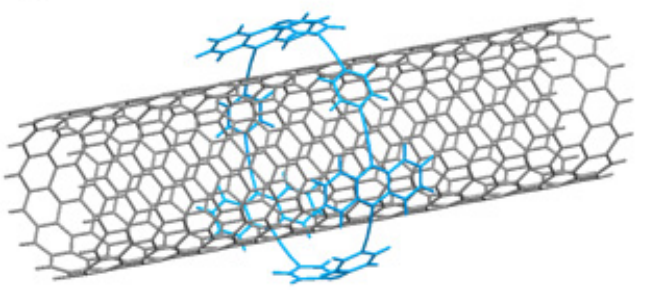

b)

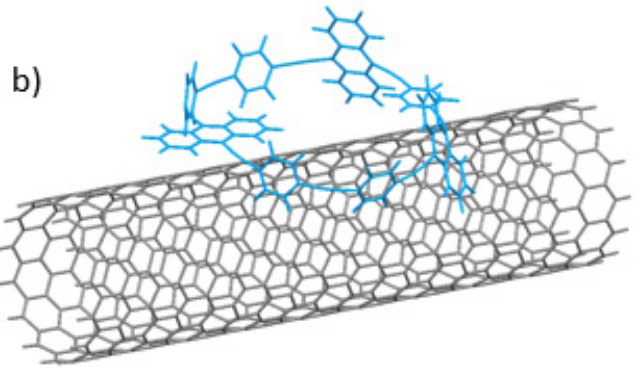

Figure S5. (a) "Tube-in-ring" and (b) "ring-on-tube" structures of CNT@CPPA complex of $(14,0)-$ CNT and 1a optimized with Forcite program and visualized by Mercury software (Mercury 3.6, provided by Cambridge Crystallographic Data Centre).

Energy-minimized structure of CNT@CPPA complex optimized by DFT calculation at $\mathrm{B} 3 \mathrm{LYP} / 6-31 \mathrm{G}(\mathrm{d})$ level is quite similar to those optimized by force field calculation with MMFF94 and Forcite program (Figure S6).
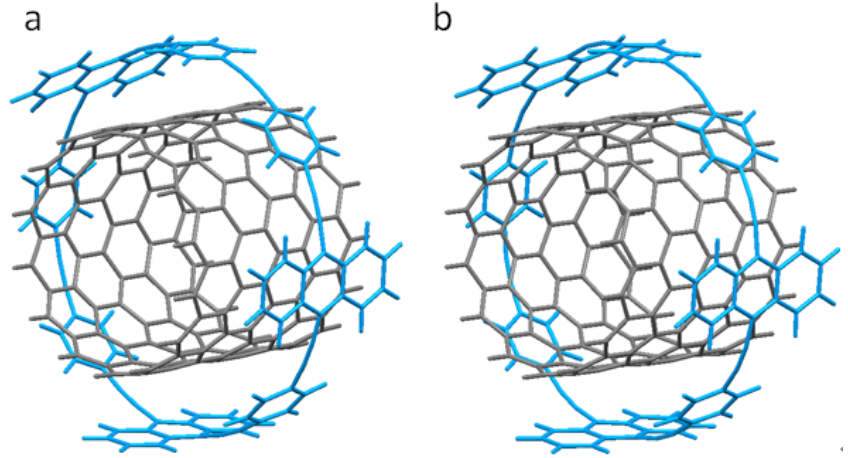

Figure S6. Energy-minimized structure of CNT@CPPA complex of $(14,0)$-CNT and 1a optimized by (a) force field calculation with Forcite and (b) DFT with B3LYP/6-31G(d) and visualized by Mercury software. 


\section{WILEY-VCH}

\subsection{Complexation of CNT with CPPAs and quantification experiment}

All single-walled carbon nanotubes (CNTs) were purified before use. A mixture of CNT (10 mg) in $30 \% \mathrm{H}_{2} \mathrm{O}_{2}$ aqueous solution $(10 \mathrm{~mL}$ ) was sonicated by ultrasonicator (bath type, $100 \mathrm{~W}, 28,45$, and $100 \mathrm{kHz}$ for each 3 seconds, W-113, Tokyo Rikakikai Co., Ltd., Japan) for $1 \mathrm{~h}$. The resulting mixture was refluxed for $8 \mathrm{~h}$ and filtered by using filter paper (pore size: $1 \mu \mathrm{m}$ ). The black solid was thoroughly washed with water (MilliQ) and dispersed in $\mathrm{CS}_{2}(5 \mathrm{~mL})$ by using ultrasonicator (10 $\left.\mathrm{min}\right)$. The resulting black solid was collected by filtration using filter paper (pore size: $1 \mu \mathrm{m}$ ), thoroughly washed with $\mathrm{CS}_{2}$, and dried at $60^{\circ} \mathrm{C}$ to afford purified CNT.

General procedure for complexation of CNT with CPPA and quantification of CNT@CPPA complexes 2 is shown in the main text. The UV-vis absorption spectra of CPPA recovered were shown in Figure $\mathrm{S} 7$.

a

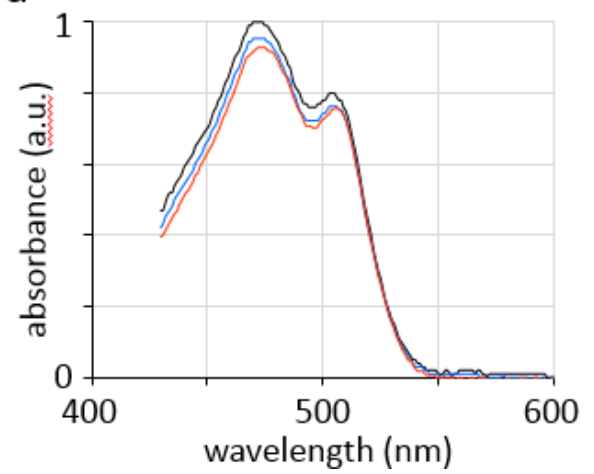

b

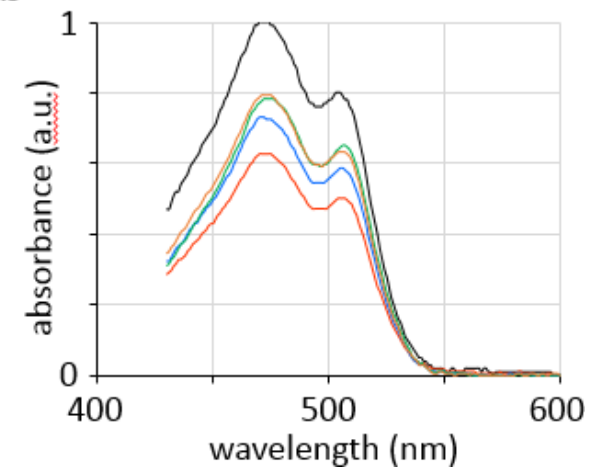

Figure S7. (a) UV-vis absorption spectra of recovered CPPA 1d treated without CNT B (black) and with 0.25 equiv of CNT B for $24 \mathrm{~h}$ (blue) and $72 \mathrm{~h}$ (red). (b) UV-vis absorption spectra of recovered CPPA 1d treated with 1.25 equiv of CNT B (orange) or 2.5 equiv of CNT A (green), CNT B (red), and CNT C (blue) for $24 \mathrm{~h}$. 


\section{WILEY-VCH}

The average distance between two CPPAs in CNT@CPPA complexes were estimated as follows.

In the case of $(11,0)$-CNT (Figure S8, diameter: $0.86 \mathrm{~nm}$, model of CNT A), 44 carbon atoms exit in the length of $0.427 \mathrm{~nm}$. This indicates that the average molecular weight of CNT A, whose diameters are $0.7-1.0 \mathrm{~nm}$, was calculated at $1230 / \mathrm{nm}$.

Similarly, average molecular weights of CNT B (approximated by $(15,0)-\mathrm{CNT}$ ) and CNT C (approximated by $(18,0)$ CNT) were estimated at $1690 / \mathrm{nm}$ and 2020/nm, respectively. Taking the amounts of complexed CPPAs into consideration, average distance between

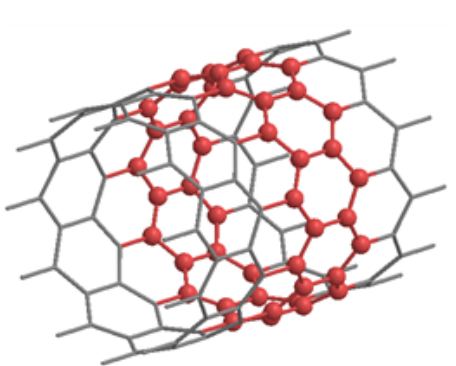

(11,0)-CNT

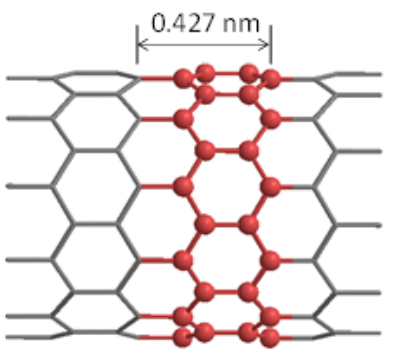

side view

Figure S8. Structure of $(11,0)$ CNT.

Table S7.

Table S7. Complexation of CPPA 1 with CNTs A-C and average distance between two CPPAs in CNT@CPPA.

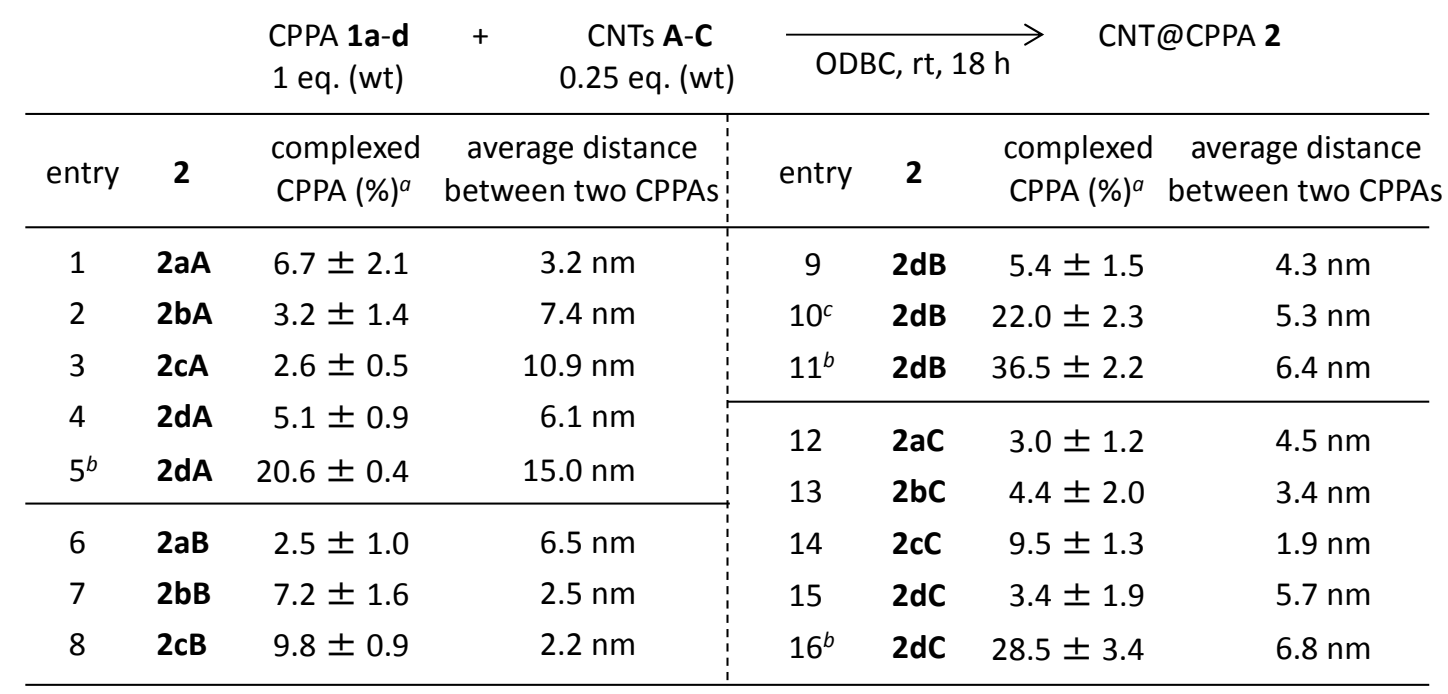

${ }^{a}$ Mean \pm s.d. $(\mathrm{n}=5) .{ }^{b} \mathrm{CNT}(2.5$ eq. $(\mathrm{wt}))$ was used. ${ }^{c} \mathrm{CNT}$ B $(1.25$ eq. $(\mathrm{wt}))$ was used. 


\section{WILEY-VCH}

\subsection{UV-vis absorption and fluorescence spectra}

The sample solutions were prepared by mixing CNT, CNT@CPPA complexes or CPPA with ODCB through sonication. UV-vis absorption spectra of CNT@CPPA complexes were measured by UV-vis spectrophotometer (V-570, JASCO Inc., Japan and UH5300, Hitachi High-Technologies Co., Japan) (Figure S9-S12). The absorbance around 400-550 $\mathrm{nm}$ which can be assigned as CPPAs were observed in UV-vis absorption spectra of all CNT@CPPA complexes. Representative fluorescence quenching through complexation (2bA-2bC) was measured by fluorescence spectrophotometer (RF-6000, Shimadzu Co., Japan) (Figure S13). This quenching indicates that the excited photoenergy of complexed CPPAs with both "tube-in-ring" and "ring-on-tube" structures could be smoothly consumed through $\pi-\pi$ interaction with CNTs.
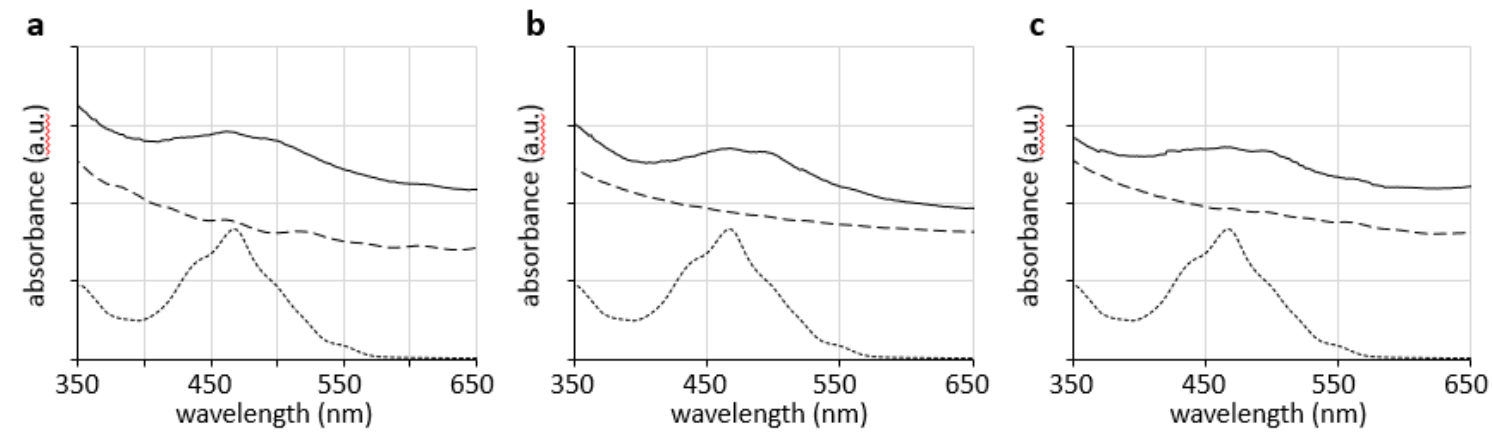

Figure S9. UV-vis absorption spectra of (a) CNT A (dashed), 2aA (solid), and 1a (dotted), (b) CNT B (dashed), 2aB (solid), and 1a (dotted), and (c) CNT C (dashed), 2aC (solid), and 1a (dotted).
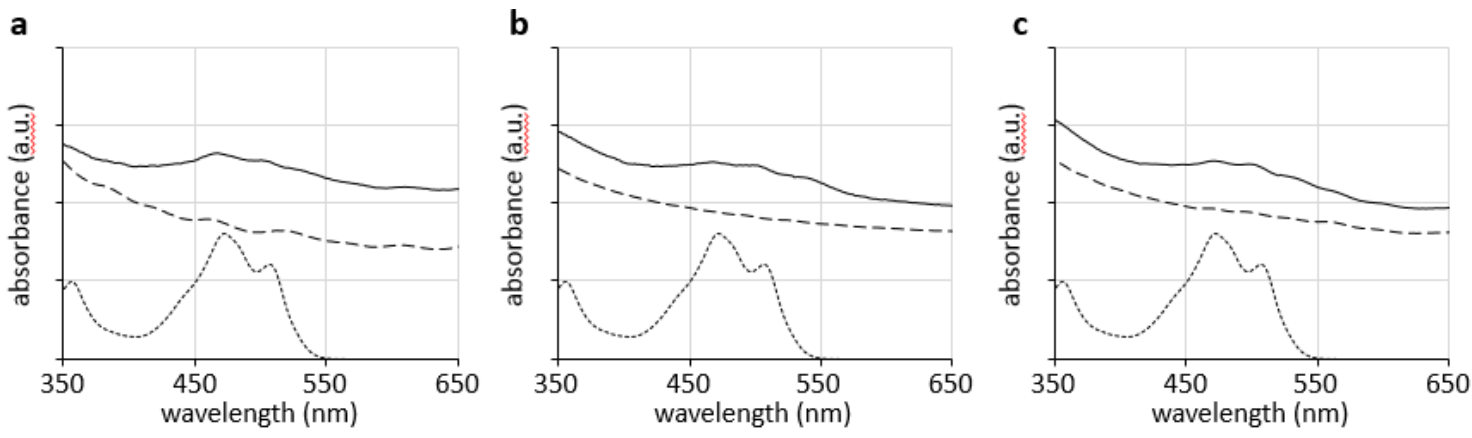

Figure S10. UV-vis absorption spectra of (a) CNT A (dashed), 2bA (solid), and 1b (dotted), (b) CNT $\mathbf{B}$ (dashed), 2bB (solid), and 1b (dotted), and (c) CNT C (dashed), 2bC (solid), and 1b (dotted). 


\section{WILEY-VCH}

a

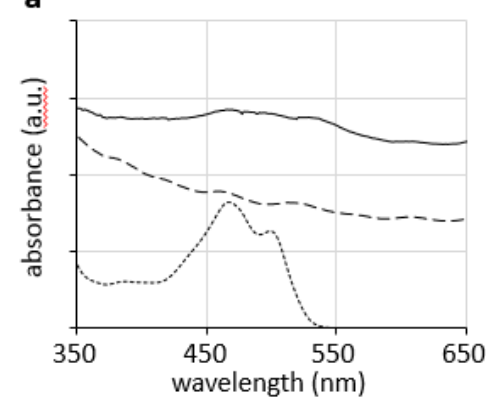

b

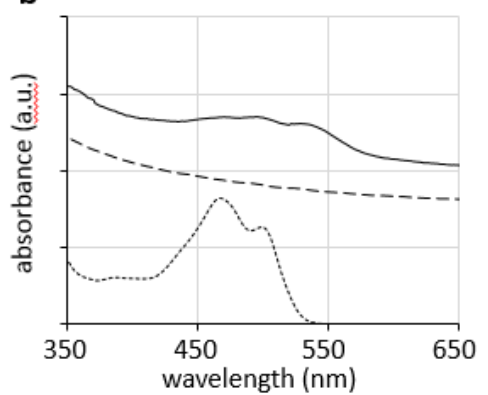

C

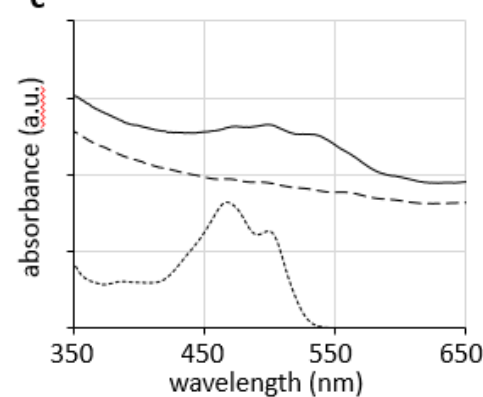

Figure S11. UV-vis absorption spectra of (a) CNT A (dashed), 2cA (solid), and 1c (dotted), (b) CNT $\mathbf{B}$ (dashed), 2cB (solid), and 1c (dotted), and (c) CNT C (dashed), 2cC (solid), and 1c (dotted).
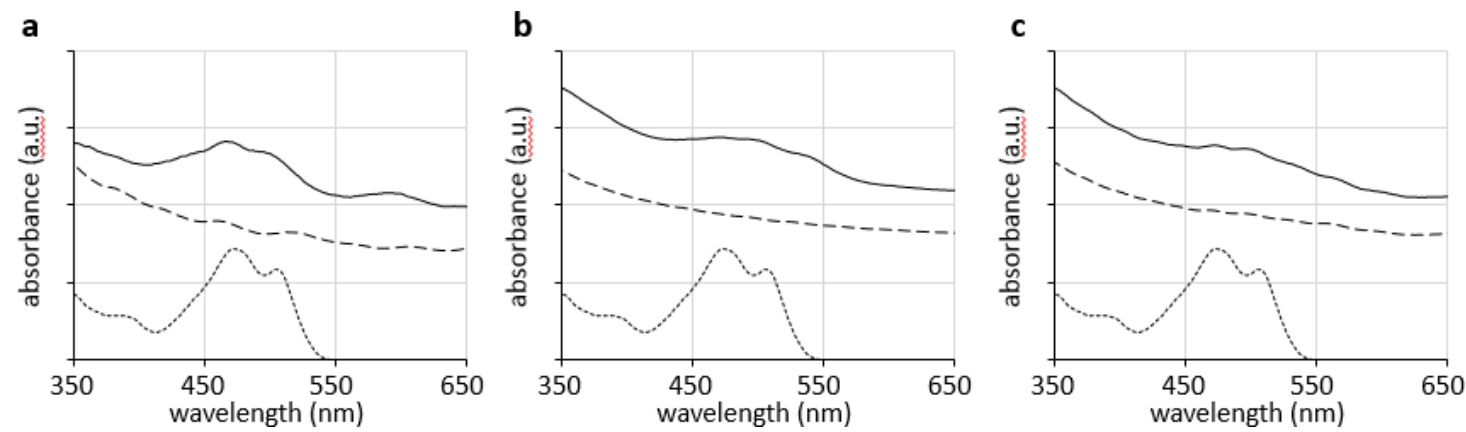

Figure S12. UV-vis absorption spectra of (a) CNT A (dashed), 2dA (solid), and 1d (dotted), (b) CNT B (dashed), 2dB (solid), and 1d (dotted), and (c) CNT C (dashed), 2dC (solid), and 1d (dotted).

a

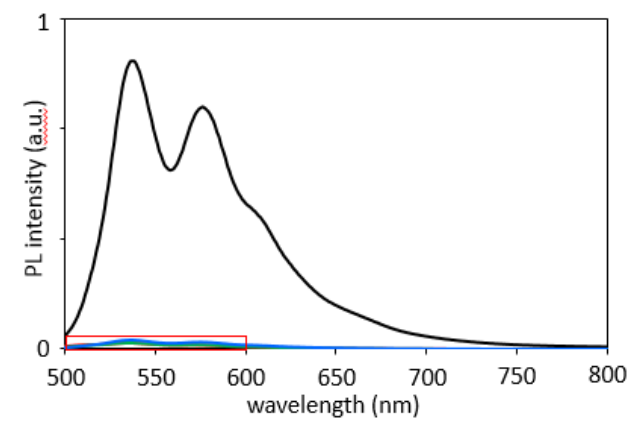

b

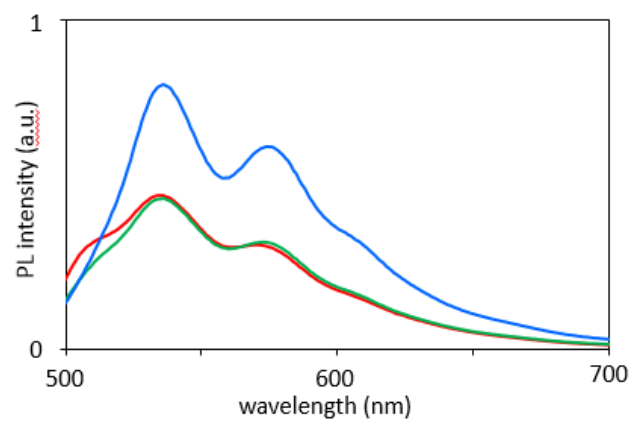

Figure S13. (a) Photoluminescence (PL) spectra of $\mathbf{2 b A}$ (red), $\mathbf{2 b B}$ (green), $\mathbf{2 b C}$ (blue), and $\mathbf{1 b}$ (black). (b) Enlarged spectra in a red square. 


\section{WILEY-VCH}

\subsection{Thermogravimetric analysis}

TGA (flow: air $(50 \mathrm{~mL} / \mathrm{min})$, scan rate: $10{ }^{\circ} \mathrm{C} / \mathrm{min}$ ) of $\mathbf{2} \mathbf{b B}$ prepared through complexation for different times was summarized in Figure S14. From the data, it was confirmed that the $72 \mathrm{~h}$ incubation was enough for preparation of CNT@CPPA complexes. TGA of 2dA-2dC were summarized in Figure S15.

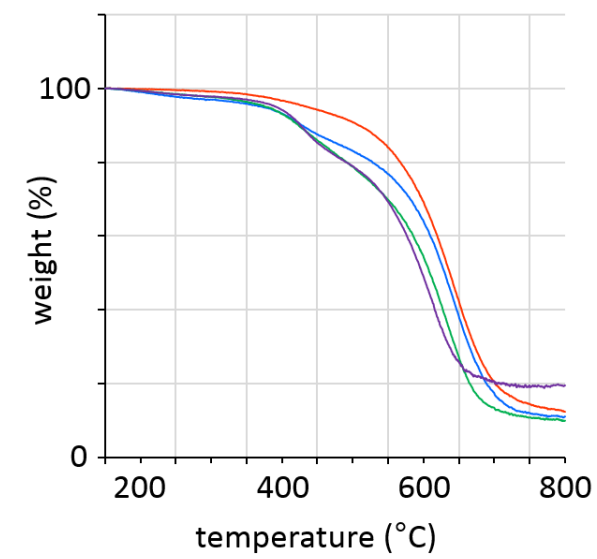

Figure S14. TGA (flow: air $\left(50 \mathrm{~mL} / \mathrm{min}\right.$ ), scan rate: $10^{\circ} \mathrm{C} / \mathrm{min}$ ) of pristine CNT B (red) and $\mathbf{2 b B}$ prepared through complexation for $24 \mathrm{~h}$ (blue), $72 \mathrm{~h}$ (green), and $120 \mathrm{~h}$ (purple).

a

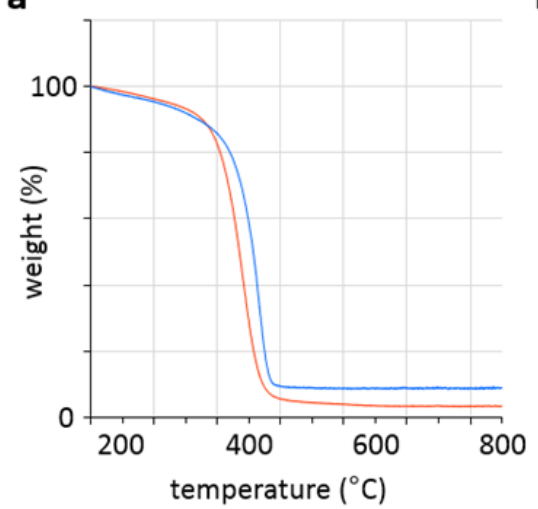

b

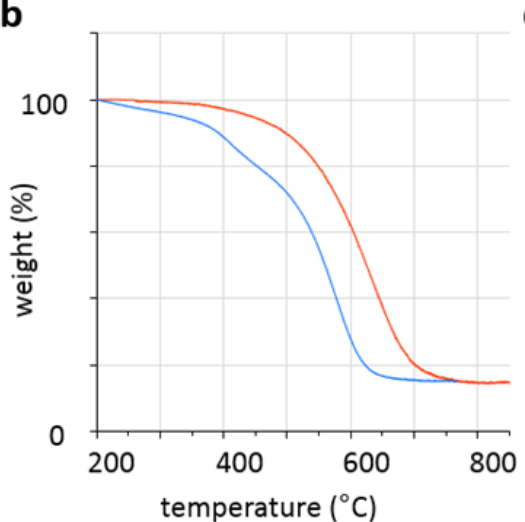

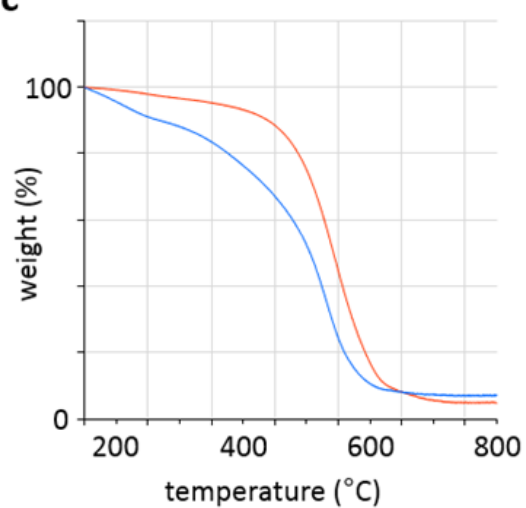

Figure S15. TGA (flow: air (50 $\mathrm{mL} / \mathrm{min}$ ), scan rate: $2{ }^{\circ} \mathrm{C} / \mathrm{min}$ ) of (a) $\mathbf{2 d A}$, (b) $\mathbf{2 d B}$, and (c) $\mathbf{2 d C}$ prepared through complexation for $24 \mathrm{~h}$. Pristine CNTs pretreated (red) and CNT@CPPAs 2 (blue). 


\section{WILEY-VCH}

\subsection{Raman spectroscopy}

Raman spectroscopy of CNT@CPPA complexes were conducted by LabRAM HR Evolution (HORIBA, Ltd.) and LabRAM ARAMIS (HORIBA, Ltd.). Raman spectra of $\mathbf{2 b A}$ and $\mathbf{2 b C}$ were measured by the former apparatus $\left(\lambda_{\mathrm{ex}}=488 \mathrm{~nm}\right)$. The spectra shown in Figure S16-S19 were measured by the latter apparatus $\left(\lambda_{\mathrm{ex}}=532 \mathrm{~nm}\right)$.

a

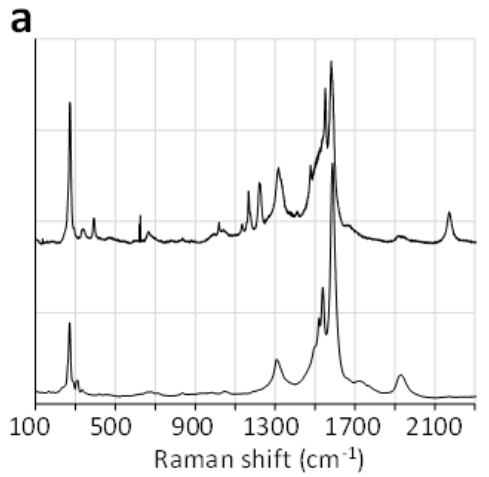

d

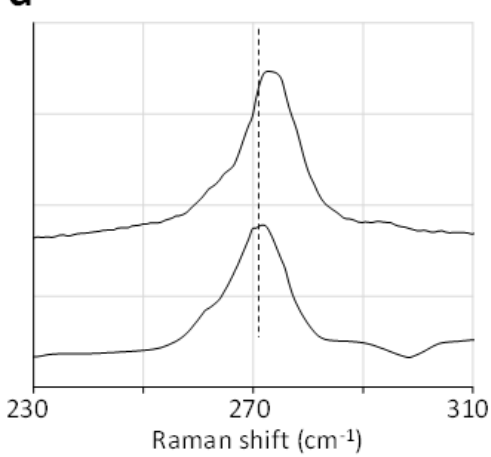

b

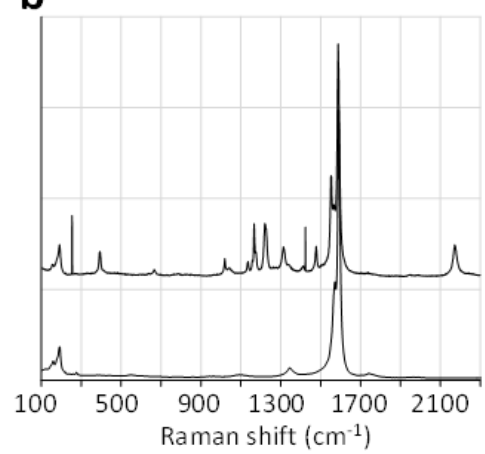

e

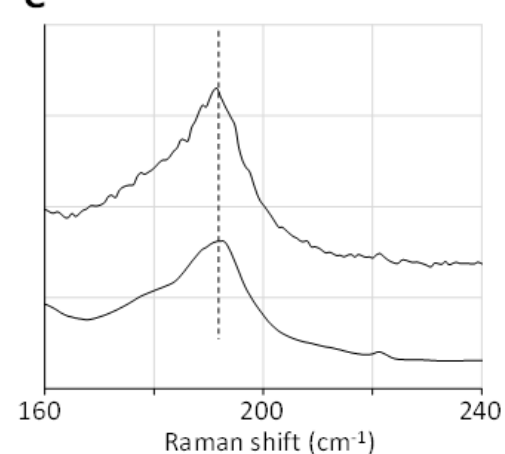

C

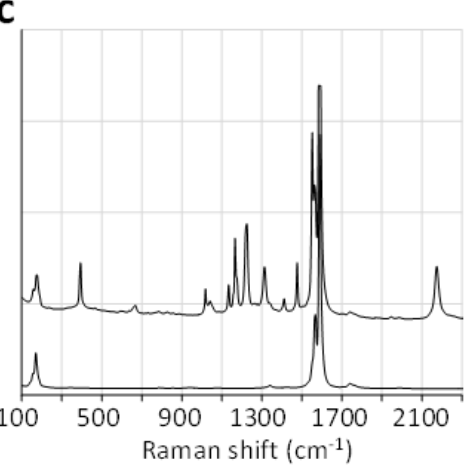

f

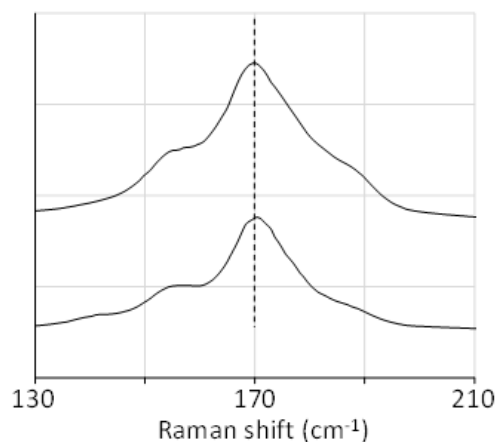

Figure S16. Raman spectra of (a) $2 \mathbf{a A}$, (b) $\mathbf{2 a B}$, and (c) $\mathbf{2 a C}$ with those of pristine CNTs (bottom). (d)-(f) Enlarged spectra of (a)-(c) around a RBM signal, respectively.

a

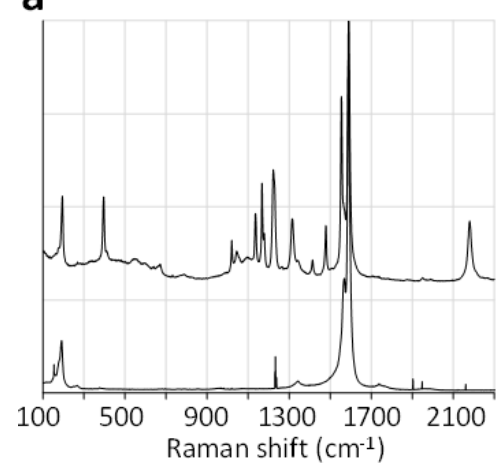

b

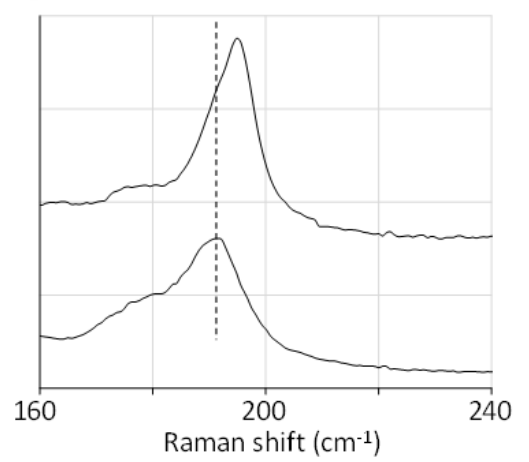

Figure S17. (a) Raman spectra of $\mathbf{2 b B}$ with that of pristine CNT B (bottom). (b) Enlarged spectra of (a) around RBM a signal. 
a

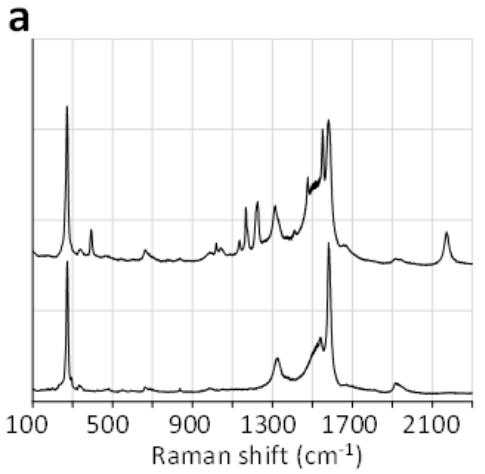

d

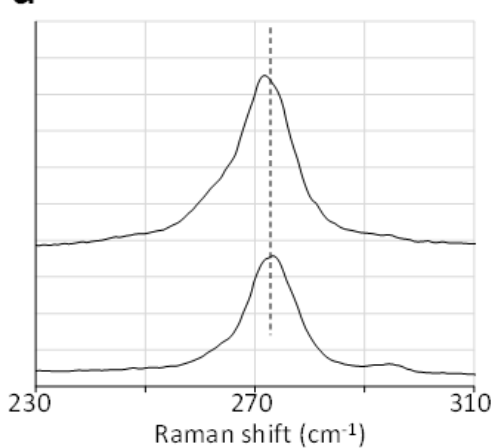

b

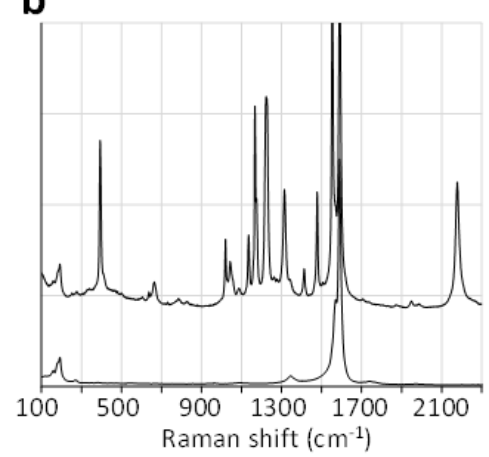

e

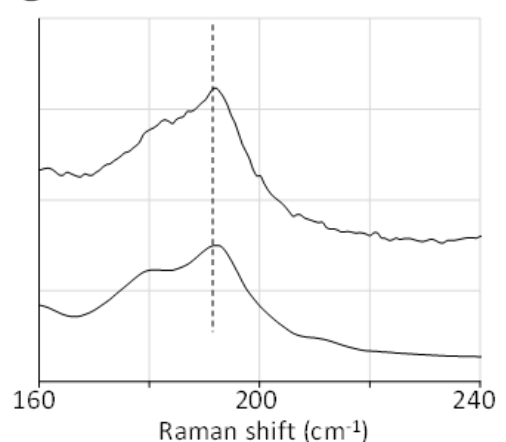

C

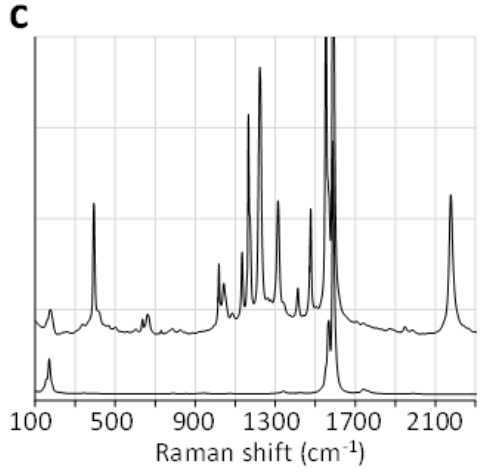

f

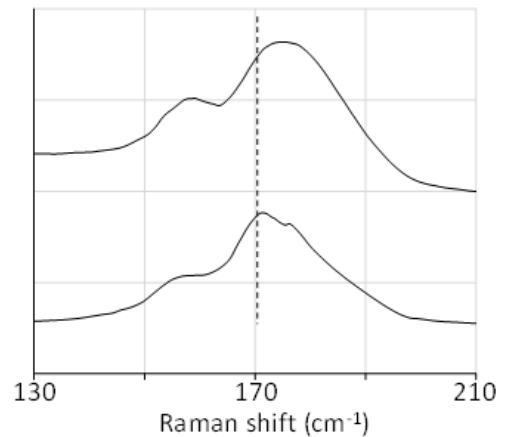

Figure S18. Raman spectra of (a) $2 \mathbf{c A}$, (b) $2 \mathbf{c B}$, and (c) $2 \mathbf{c C}$ with those of pristine CNTs (bottom). (d)-(f) Enlarged spectra of (a)-(c) around a RBM signal, respectively.

a

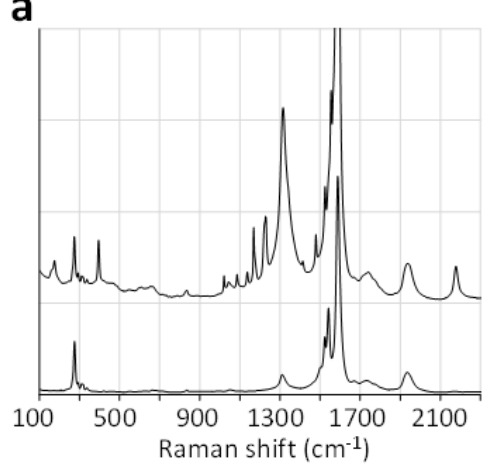

d

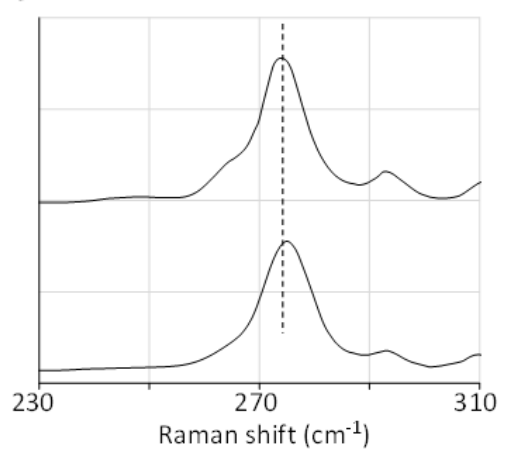

b

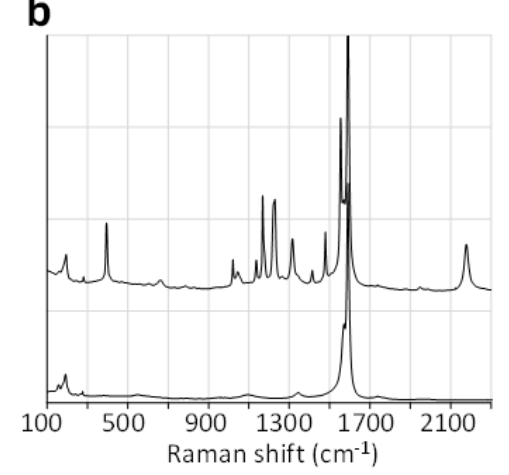

e

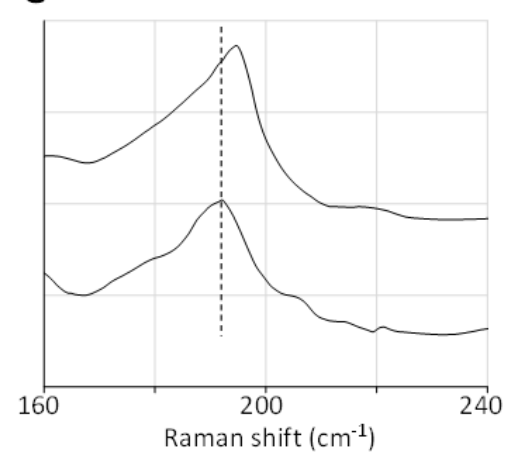

WILEY-VCH 


\section{WILEY-VCH}

\subsection{TEM observation}

TEM (accelerating voltage: 120 kV, JEM-1400, JEOL Ltd., Japan and 160 kV, JEM-2100F(HR), JEOL Ltd., Japan) was used to visualize the morphology of CNT@CPPA complexes. The merged image shown in Figure S20d indicates that the optimized structure of CNT@CPPA complex of $(17,0)$ CNT and two 1b molecules shows good agreement with TEM image. Interestingly, CNT with larger diameter $(\phi=\sim 2.4 \mathrm{~nm})$ could not interact with CPPA 1b to form "ring-on-tube"-type complexes, because the more flattened structure of $\mathbf{1 b}$ is necessary for complexation but unfavorable.

In the case of the complexation of CNT of larger diameter, no "tube-in-ring" or "ring-on-tube" complex was observed (Figure S20e). The "ring-on-tube" complexation of CNT with CPPA of an unstable flattened structure might be suppressed.

a
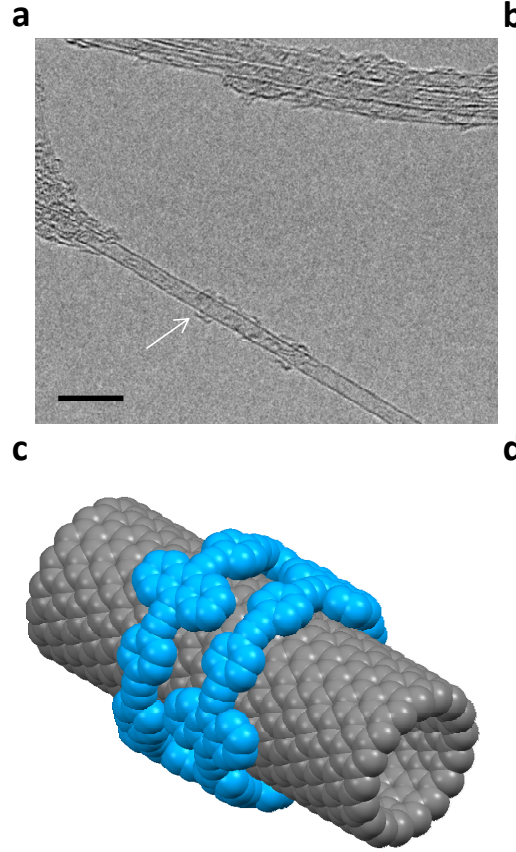

e

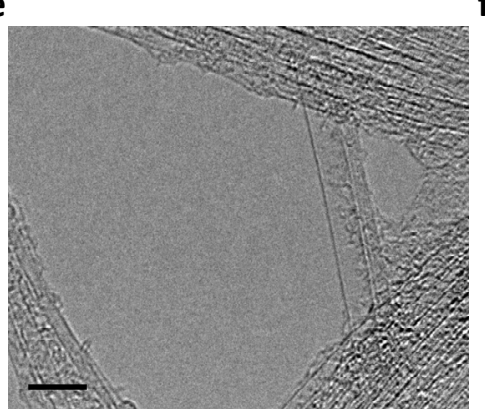

b
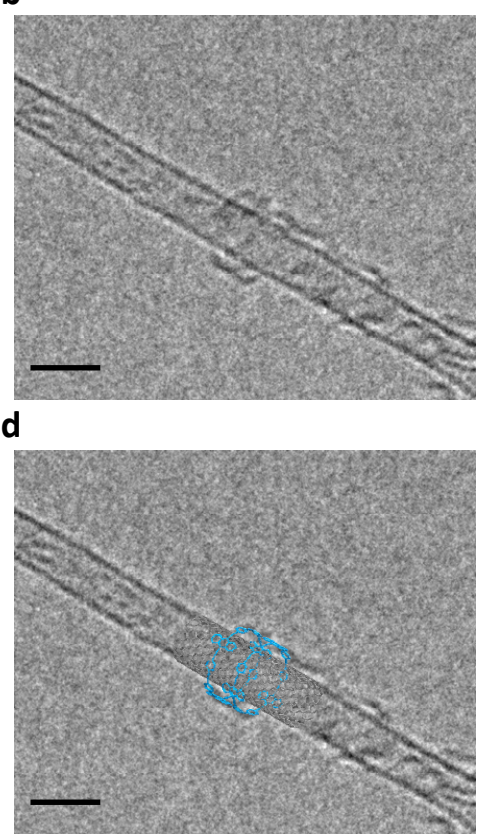

f

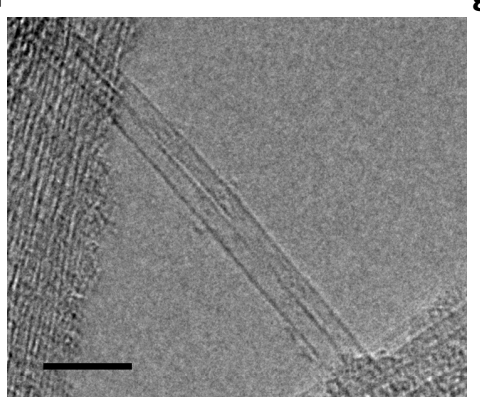

g

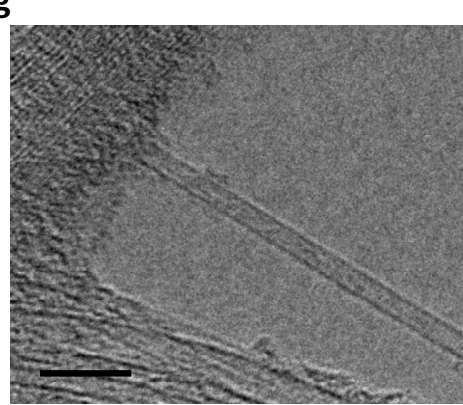

Figure S20. (a) Representative TEM image of 2bC. Scale bars, $5 \mathrm{~nm}$. (b) Enlarged image of Figure S20a. Scale bars, 2 nm. (c) Capped stick model of CNT@CPPA complex of (17,0)-CNT and two 1b molecules optimized with Forcite program and visualized by Mercury software (Mercury 3.6, provided by Cambridge Crystallographic Data Centre). Hydrogen atoms were omitted for clarity. (d) Merged image. Capped stick model of CNT@CPPA complex (Figure S20b) was merged with TEM image shown in Figure S20a. Scale bars, $2 \mathrm{~nm}$. (e) Representative TEM image of $\mathbf{2 b C}$ containing CNT with larger diameter. Scale bars, $5 \mathrm{~nm}$. (f,g) Representative TEM images of CNT C after pretreatment. Scale bar, $5 \mathrm{~nm}$. 


\section{WILEY-VCH}

\subsection{Scanning probe microscope}

SPM images of pretreated CNTs A and $\mathbf{B}$ were summarized in Figure S21. The surface of CNTs are flat and smooth, and the remnants were scarcely observed after pretreatment. "Tube-inring" structures were observed in SPM images of $\mathbf{2 b B}$ (Figure 7e and Figure S22). "Ring-on-tube" structures were also observed in same images (Figure S22i and S22j).

a

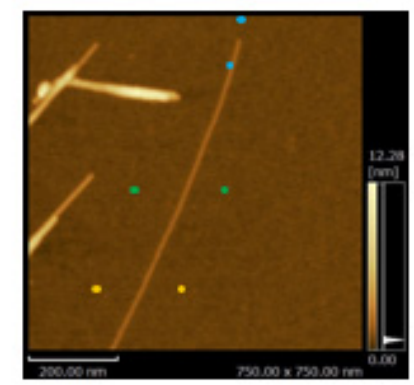

b

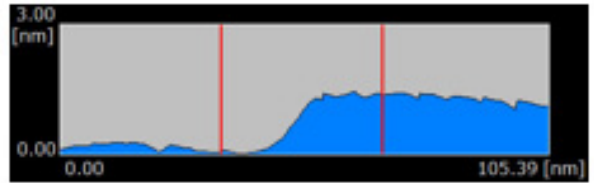

height: 1.31 nm (red)

C

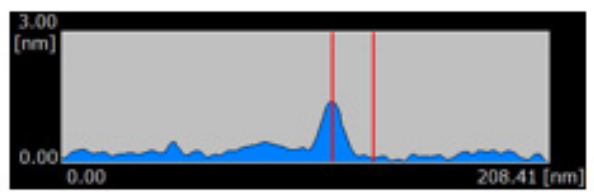

d

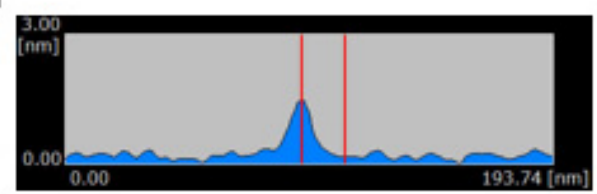

height: $1.32 \mathrm{~nm}$ (red)

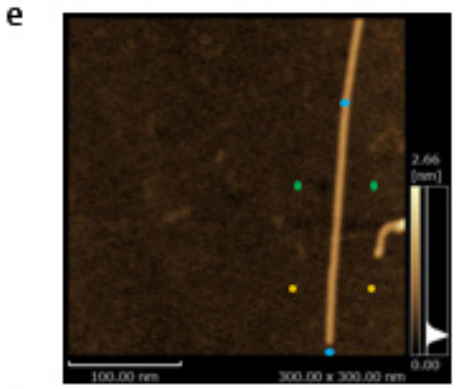

f

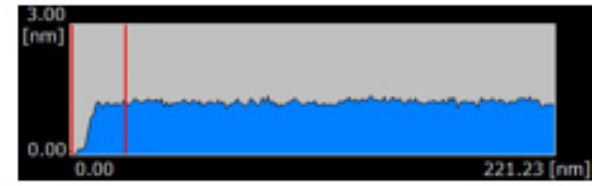

height: $1.18 \mathrm{~nm}$ (red)

g

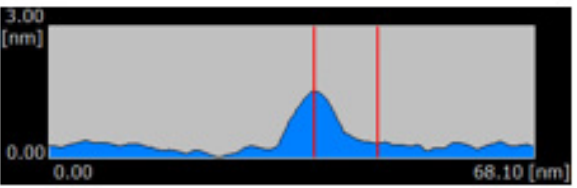

height: $1.18 \mathrm{~nm}$ (red)

h

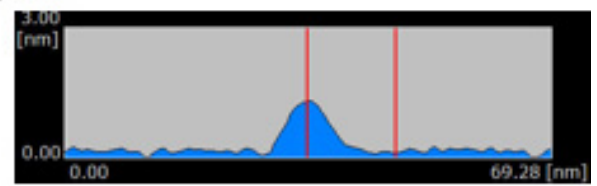

height: $1.19 \mathrm{~nm}$ (red)

Figure S21. (a) Representative SPM image of pretreated CNT B (scale bar: $200 \mathrm{~nm}$ ). Height profiles between two (b) blue, (c) green, and (d) yellow spots, respectively. (e) SPM image of pretreated CNT A (scale bar: $100 \mathrm{~nm}$ ). Height profiles between two (f) blue, (g) green, and (h) yellow spots. 


\section{WILEY-VCH}

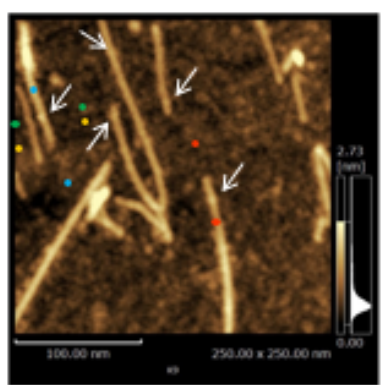

b

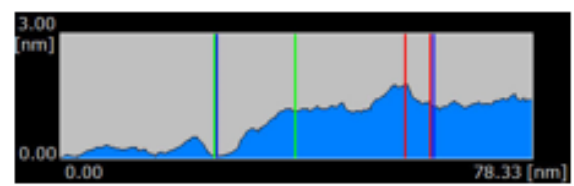

height: $0.54 \mathrm{~nm}$ (red), $1.13 \mathrm{~nm}$ (green), $1.24 \mathrm{~nm}$ (blue)

f

g
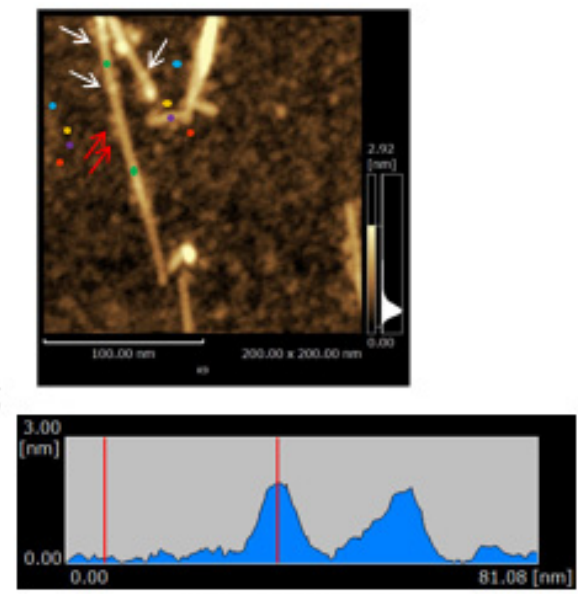

h

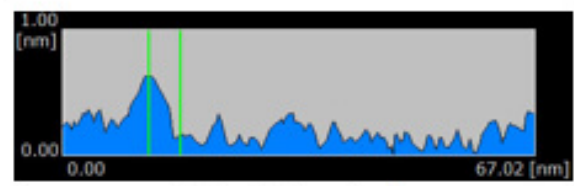

height: $0.47 \mathrm{~nm}$ (red) c

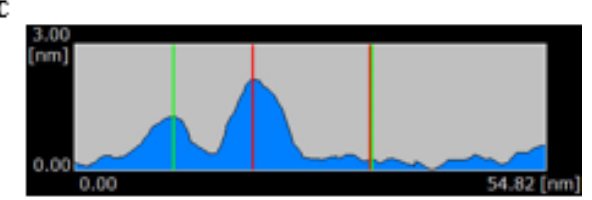

d

height: $1.93 \mathrm{~nm}$ (red), $1.06 \mathrm{~nm}$ (green)

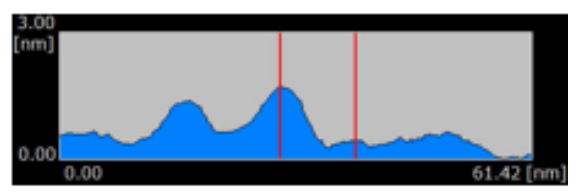

e

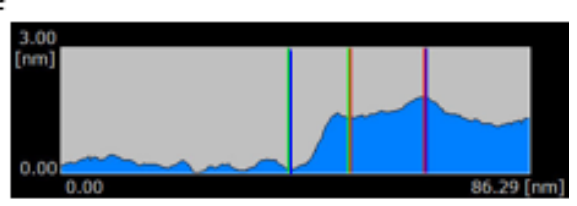

height: $0.50 \mathrm{~nm}$ (red), $1.26 \mathrm{~nm}$ (green), $1.76 \mathrm{~nm}$ (blue)

i

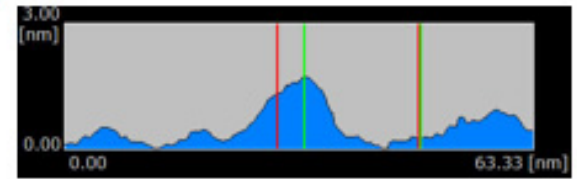

j

height: $1.06 \mathrm{~nm}$ (red), $1.48 \mathrm{~nm}$ (green)

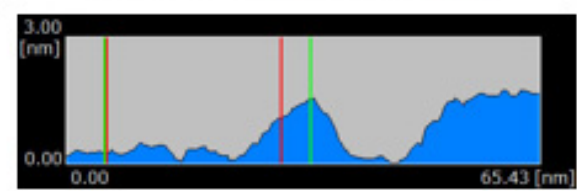

height: $0.85 \mathrm{~nm}$ (red), $1.29 \mathrm{~nm}$ (green)

k

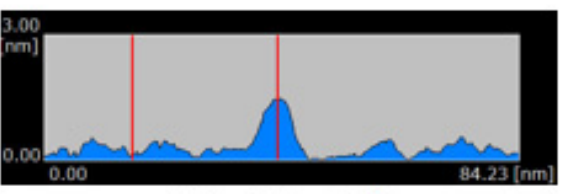

height: $1.33 \mathrm{~nm}$ (red)

Figure S22. (a) Representative SPM image of $\mathbf{2 b B}$ (scale bar: $100 \mathrm{~nm}$ ). Height profiles between two (b) blue, (c) green, (d) yellow, and (e) red spots, respectively. (f) Representative SPM image of $\mathbf{2 b B}$ (scale bar: $100 \mathrm{~nm}$ ). Height profiles between two $(\mathrm{g})$ blue, $(\mathrm{h})$ green, (i) yellow, (j) purple, and $(\mathrm{k})$ red spots. White arrows: "tube-in-ring"-type structures; red arrows: a ring obliquely attached on a tube. 


\section{WILEY-VCH}

\section{References}

1) Miki, K.; Matsushita, T.; Inoue, Y.; Senda, Y.; Kowada, T.; Ohe, K. Chem. Commun. 2013, 49, 9092.

2) Orita, A.; Taniguchi, H.; Otera, J. Chem. Asian J. 2006, 1, 430.

3) Huang, X.; Meng, J.; Dong, Y.; Cheng, Y.; Zhu, C. Polymer 2010, 51, 3064.

4) Rose, B. D.; Maria, P. J. S.; Fix, A. G.; Vonnegut, C. L.; Zakharov, L. N.; Parkin, S. R.; Haley, M. M. Beilstein J. Org. Chem. 2014, 10, 2122.

5) Wessig, P.; Gerngroß, M.; Freyse, D.; Bruhns, P.; Przezdziak, M.; Schilde, U.; Kelling, A. J. Org. Chem. 2016, 81, 1125.

6) V. K Patel; E. Kayahara; S. Yamago, Chem. Eur. J. 2015, 21, 5742-5749. 
5. NMR spectra

${ }^{1} \mathrm{H}$ NMR spectrum of $\mathbf{1 a}$

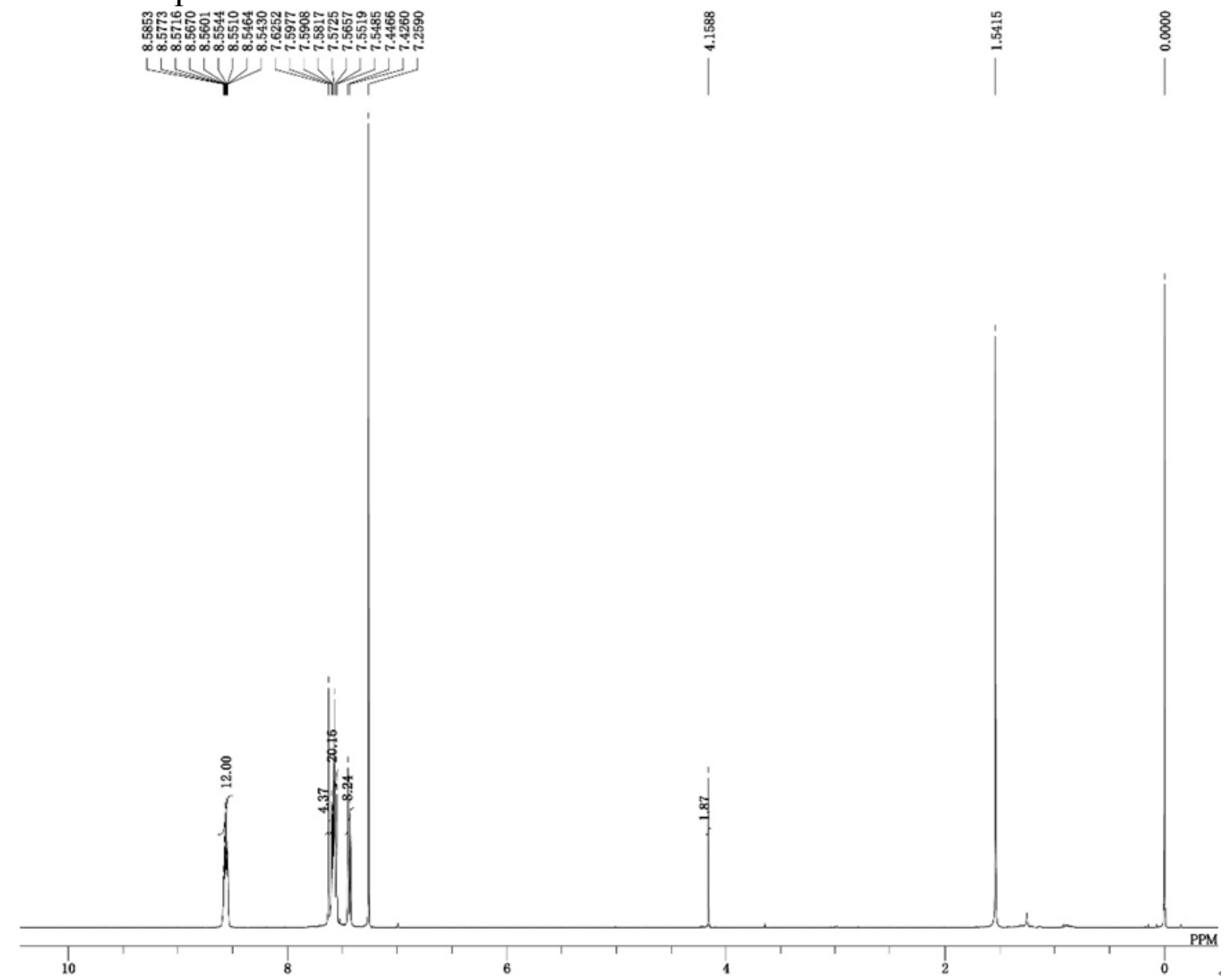

${ }^{13} \mathrm{C}$ NMR spectrum of $\mathbf{1 a}$

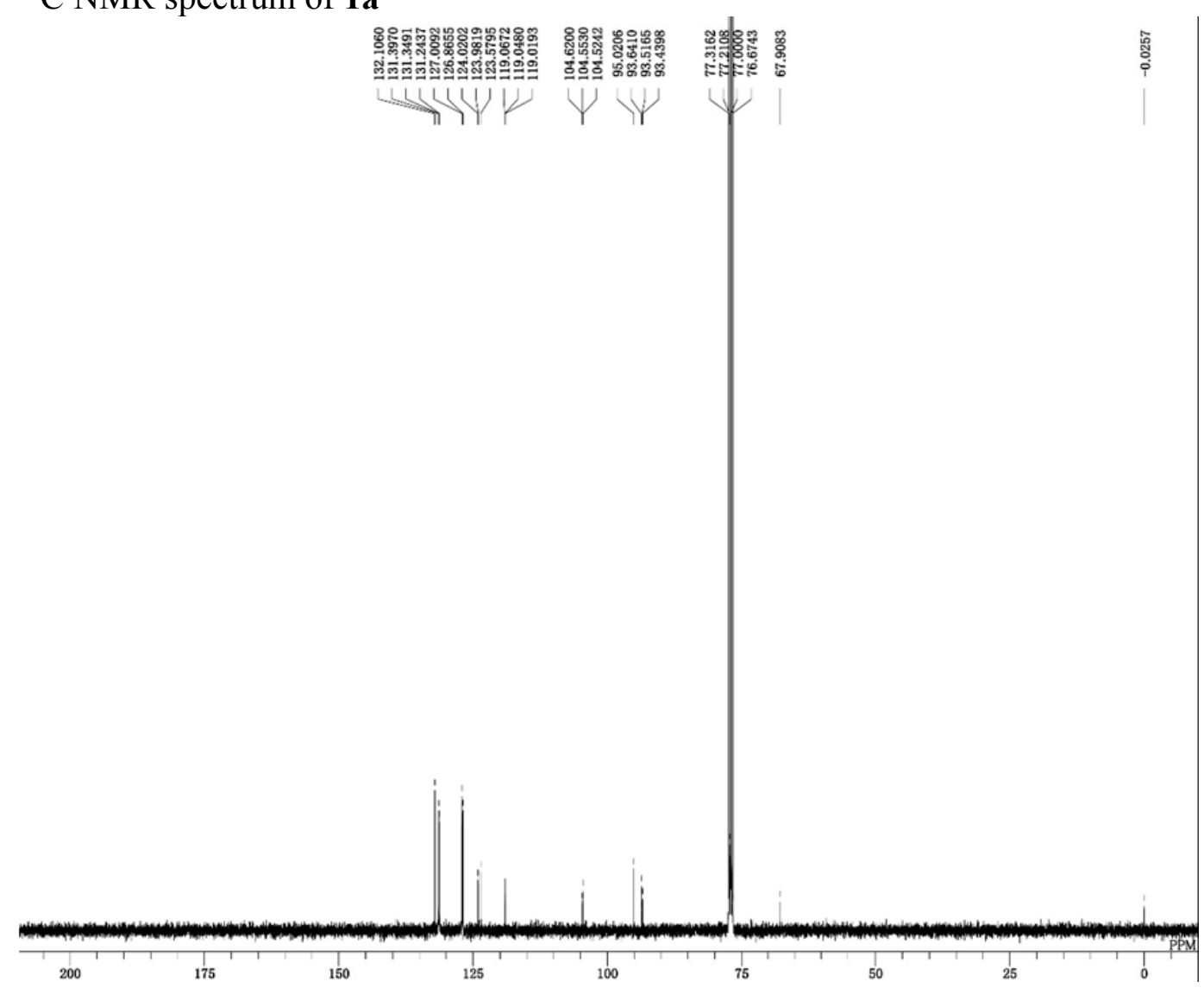




\section{WILEY-VCH}

${ }^{1} \mathrm{H}$ NMR spectrum of $\mathbf{1 b}$

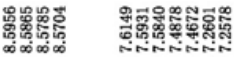

Y YilY

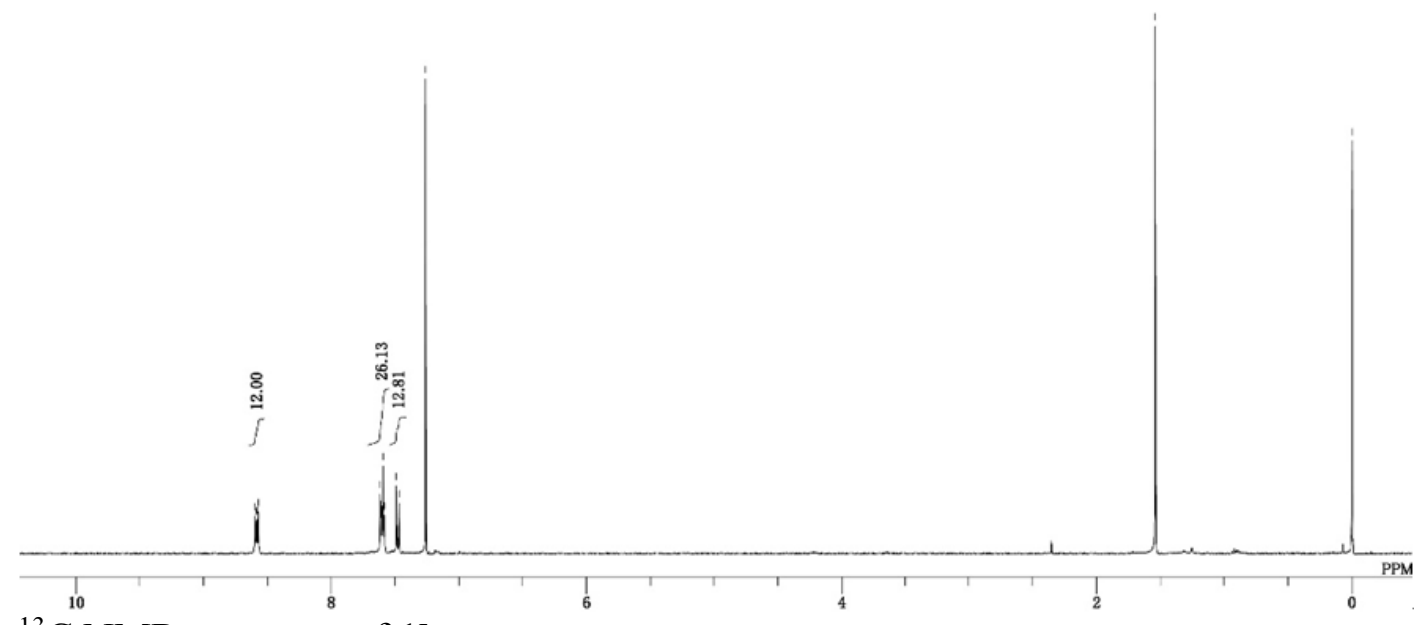

${ }^{13} \mathrm{C}$ NMR spectrum of $\mathbf{1 b}$

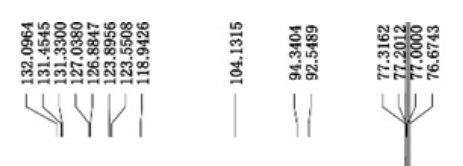

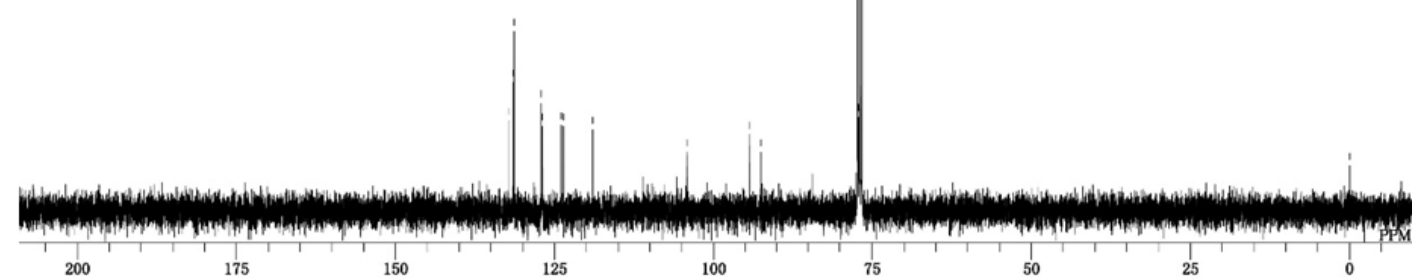




\section{WILEY-VCH}

${ }^{1} \mathrm{H}$ NMR spectrum of $1 \mathrm{c}$

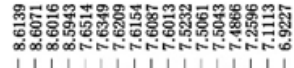

$\psi$ W
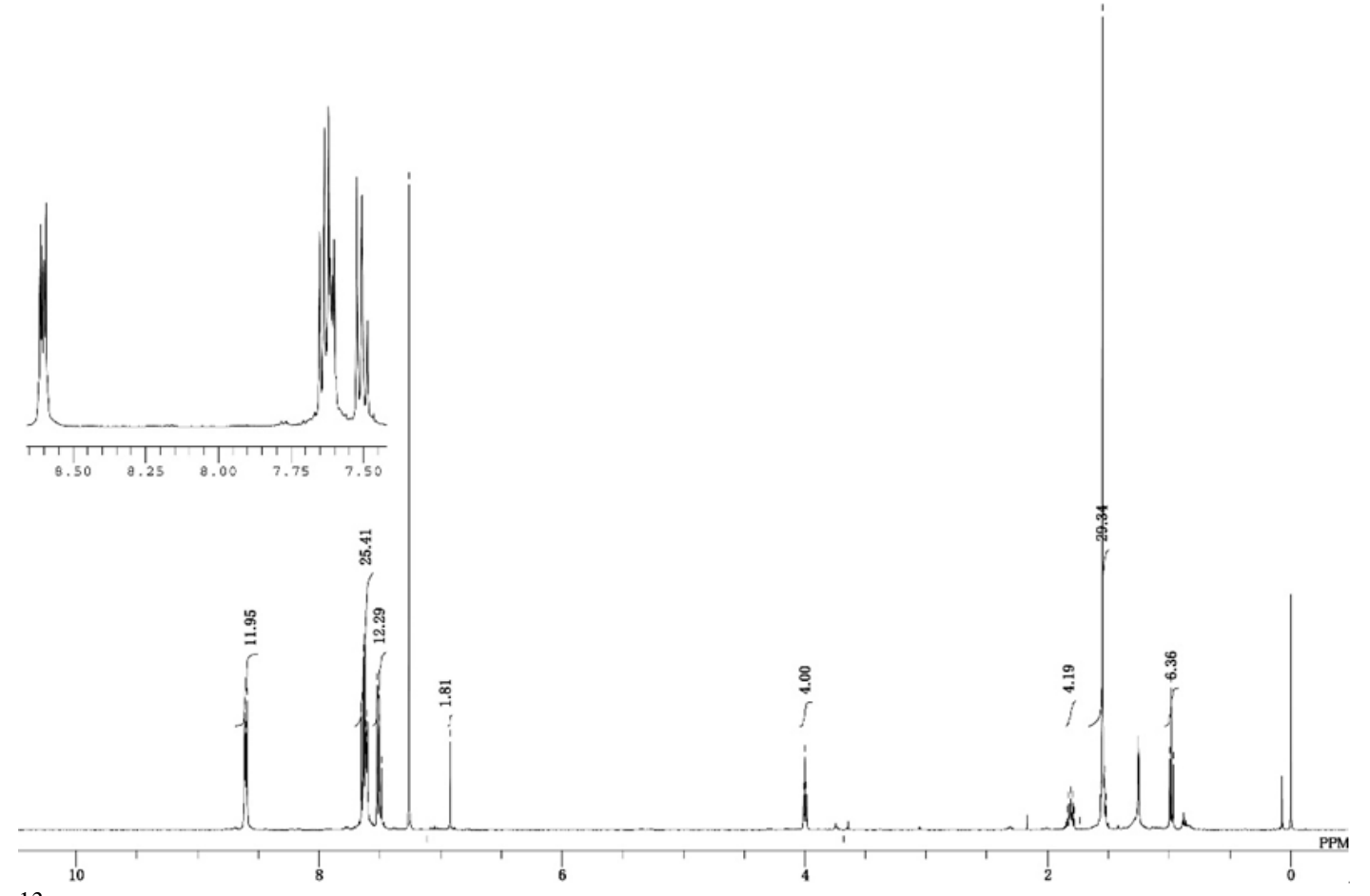

${ }^{13} \mathrm{C}$ NMR spectrum of $\mathbf{1 c}$

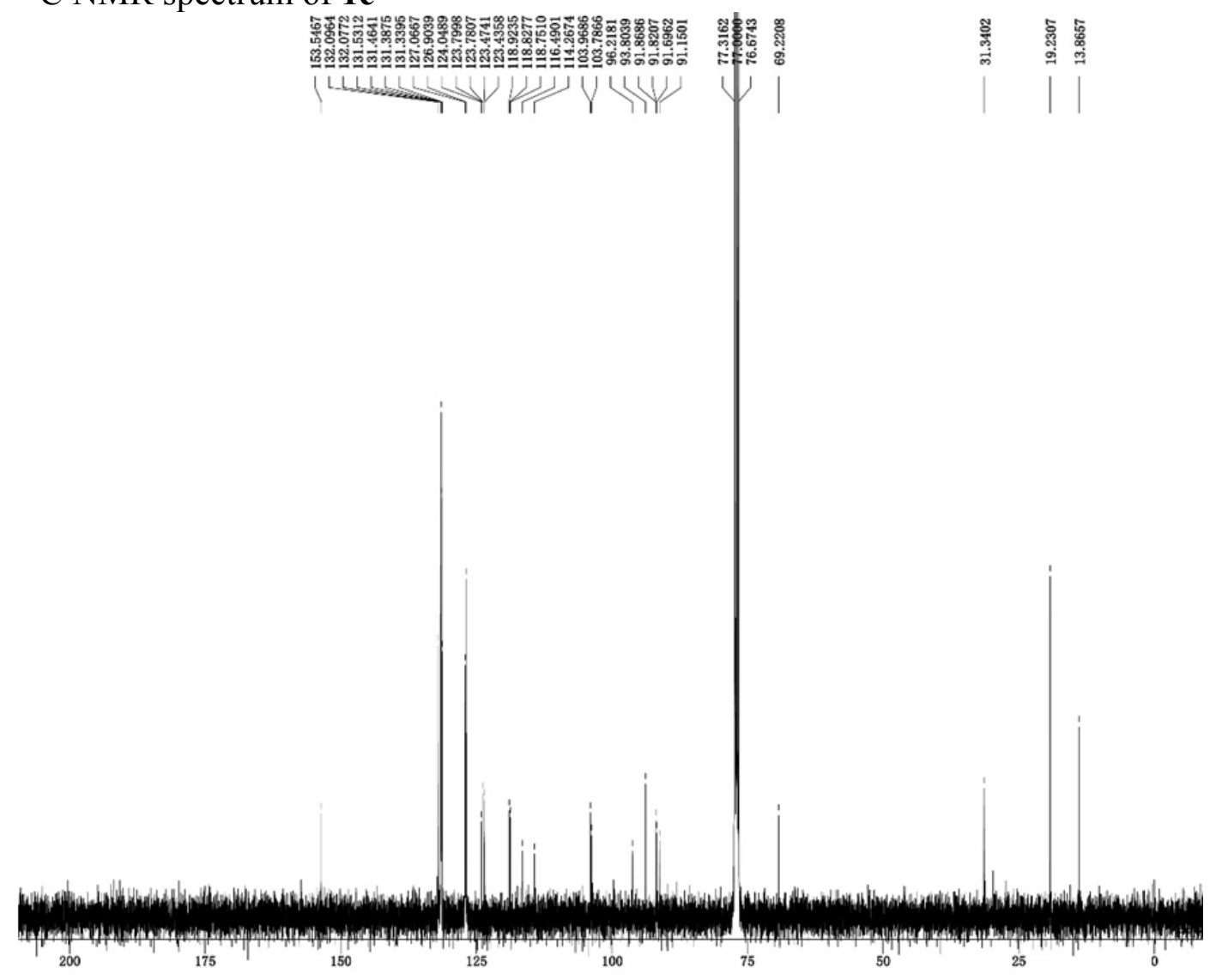




\section{WILEY-VCH}

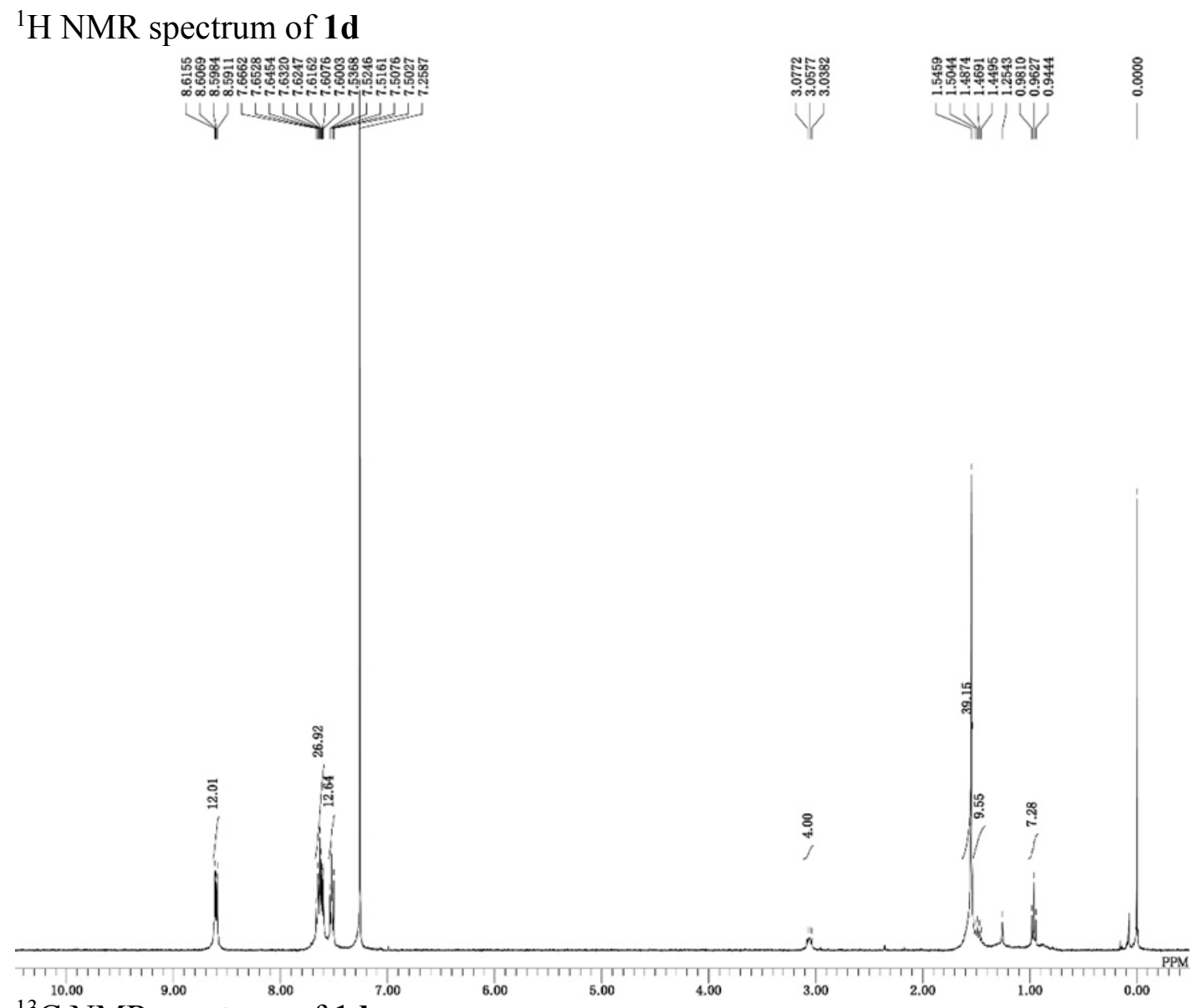

${ }^{13} \mathrm{C}$ NMR spectrum of $\mathbf{1 d}$
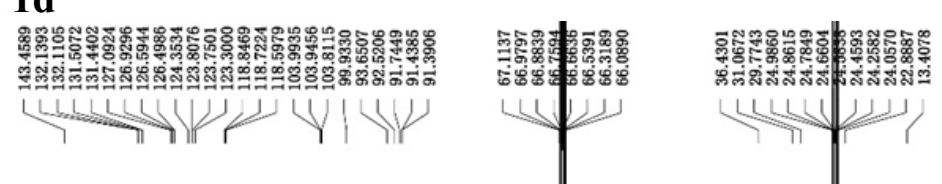
WILEY-VCH

${ }^{1} \mathrm{H}$ NMR spectrum of $\mathbf{3}$

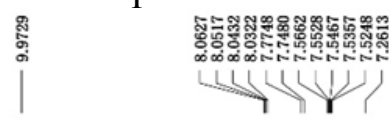

|

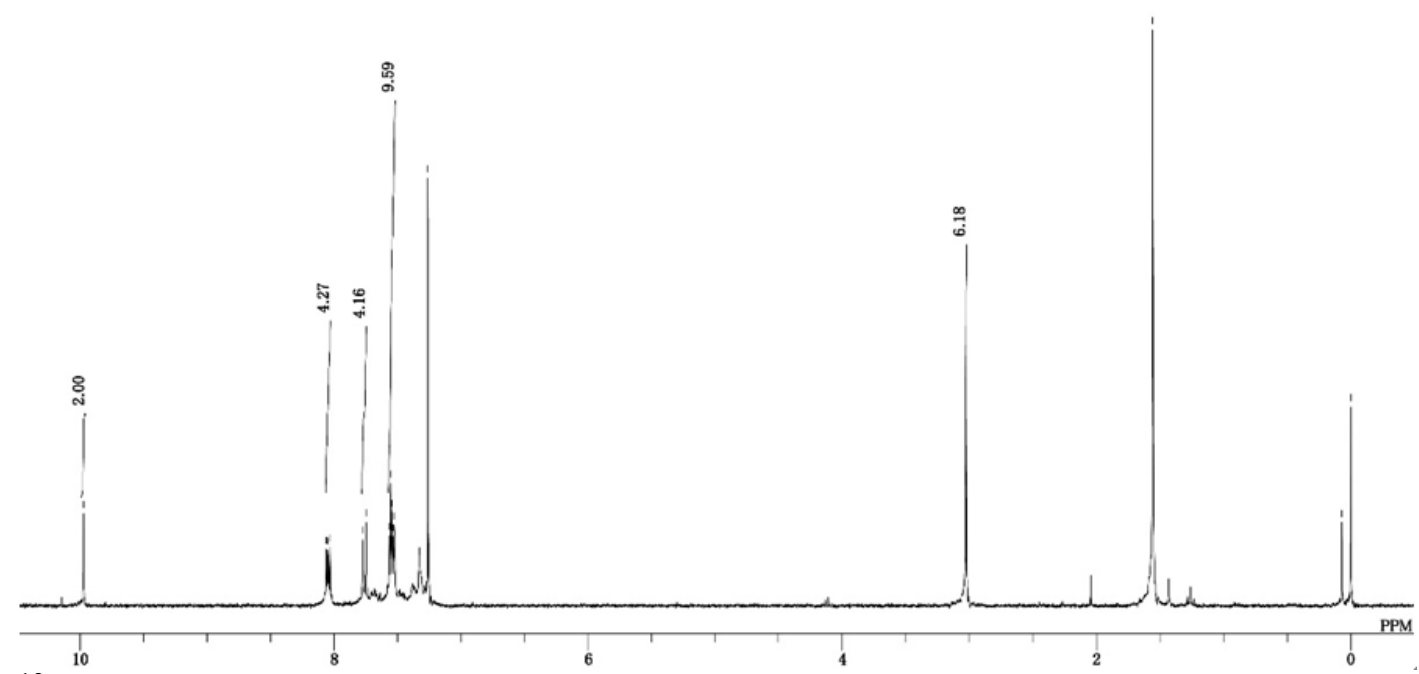

${ }^{13} \mathrm{C}$ NMR spectrum of $\mathbf{3}$

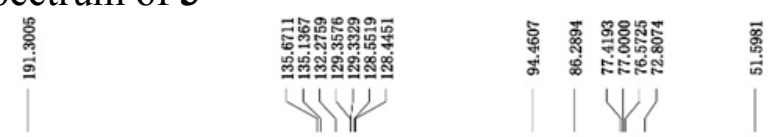

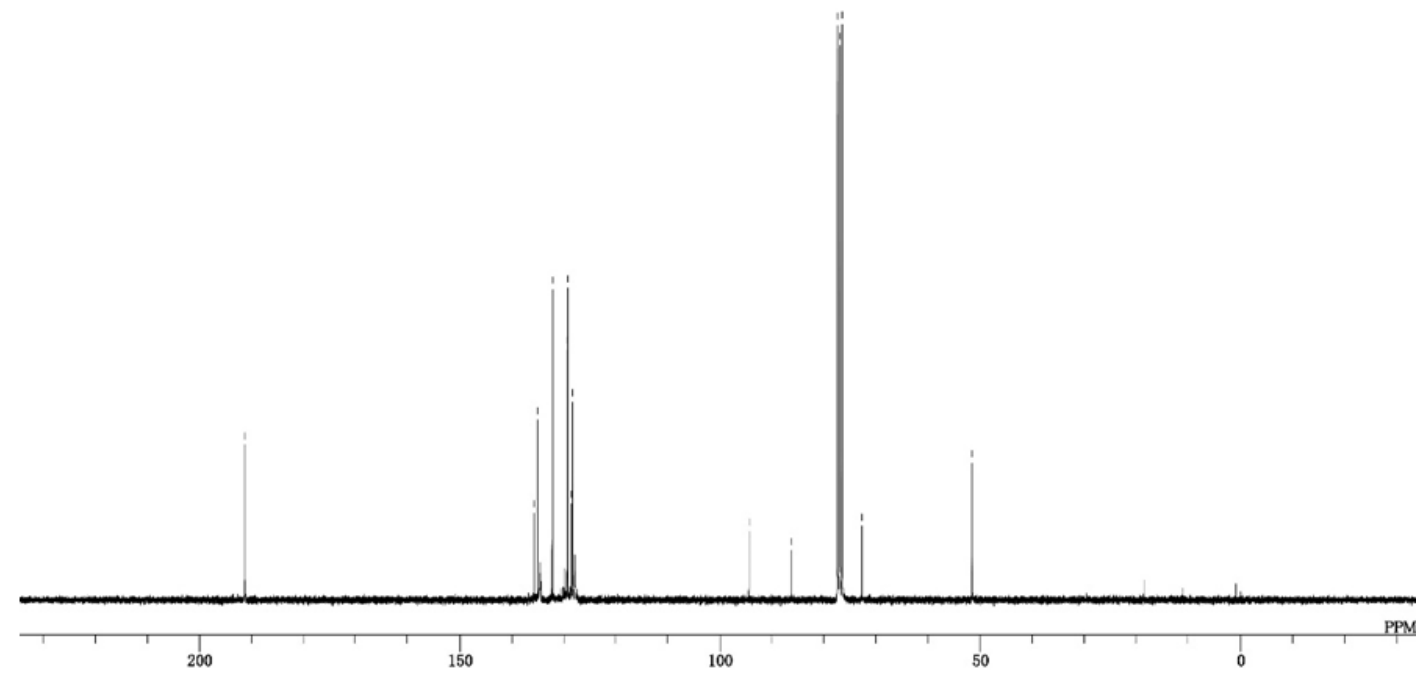




\section{WILEY-VCH}

${ }^{1} \mathrm{H}$ NMR spectrum of 4

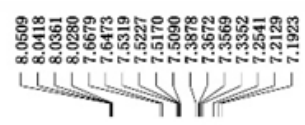

|

祭

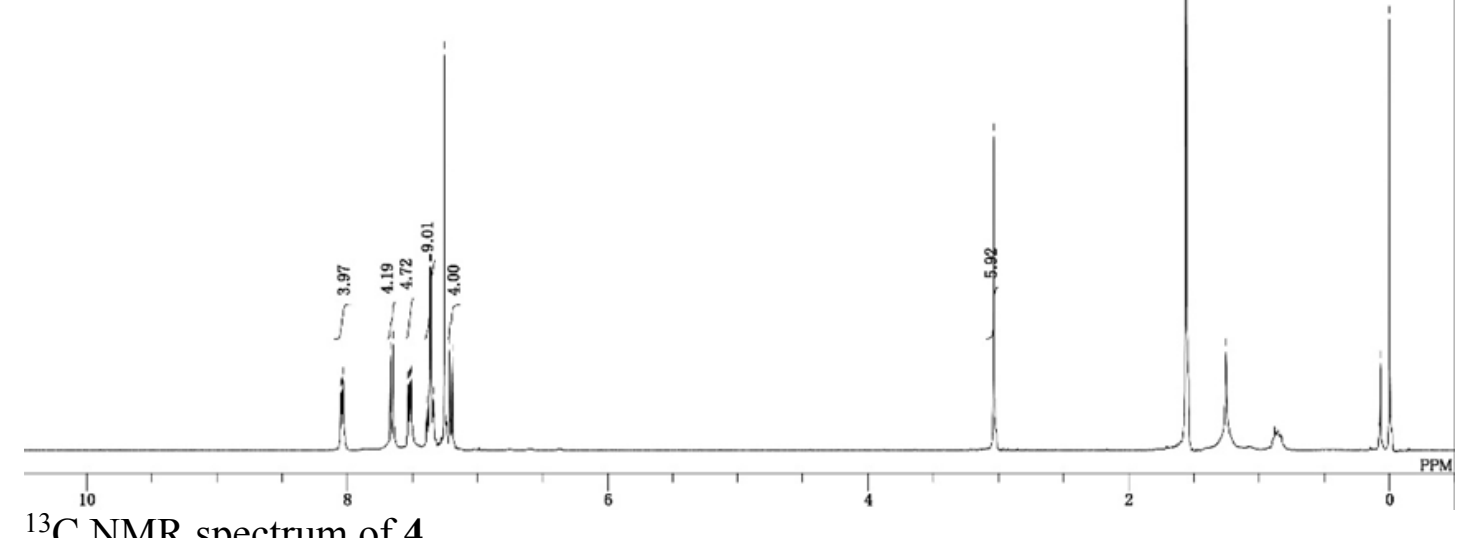

${ }^{13} \mathrm{C}$ NMR spectrum of 4

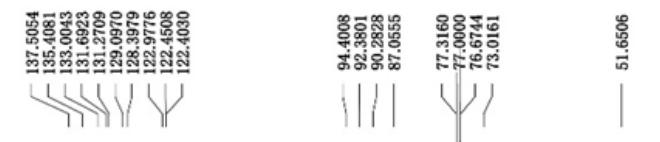

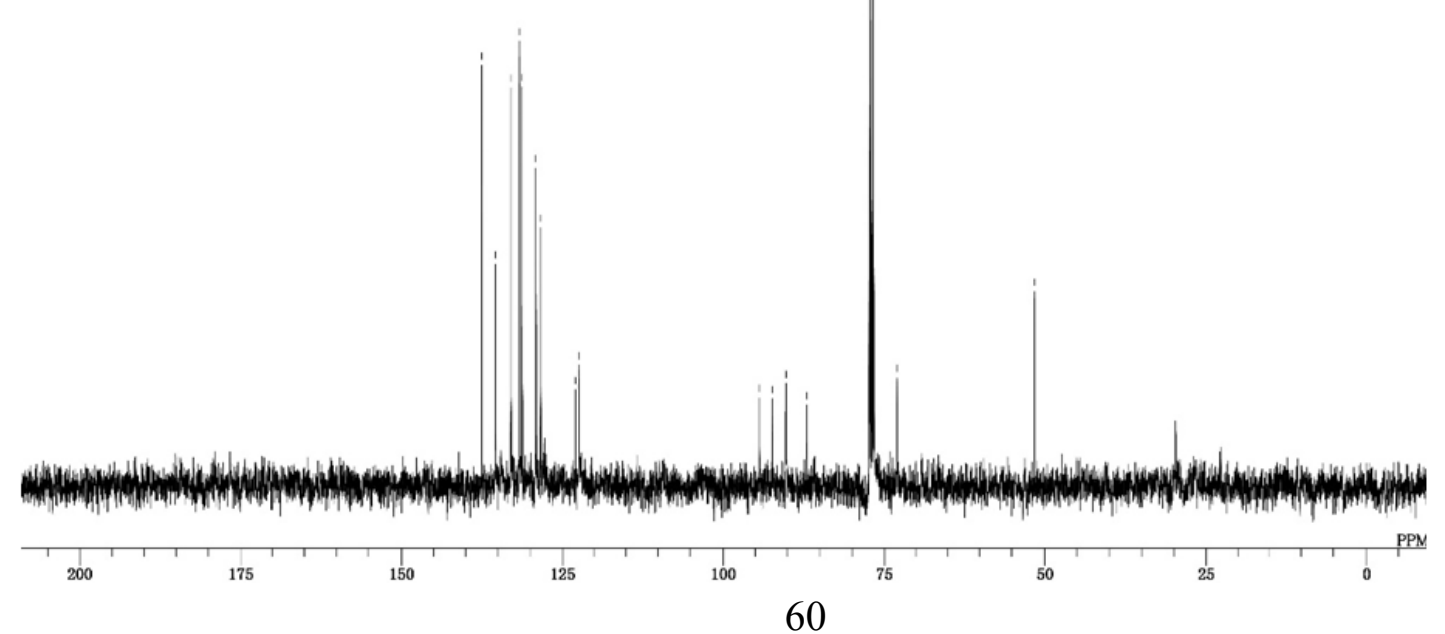




\section{WILEY-VCH}

${ }^{1} \mathrm{H}$ NMR spectrum of $\mathbf{5}$

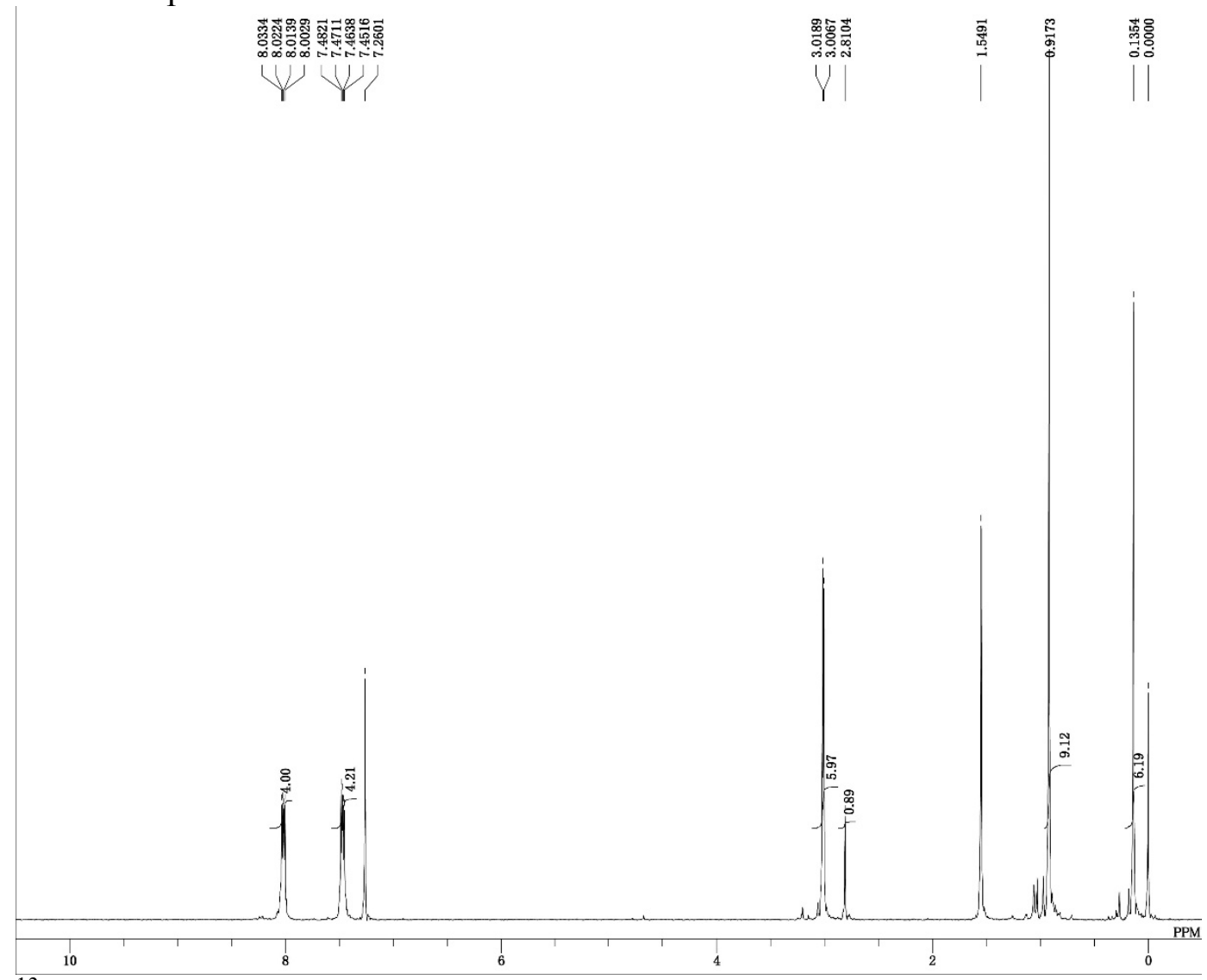

${ }^{13} \mathrm{C}$ NMR spectrum of 5

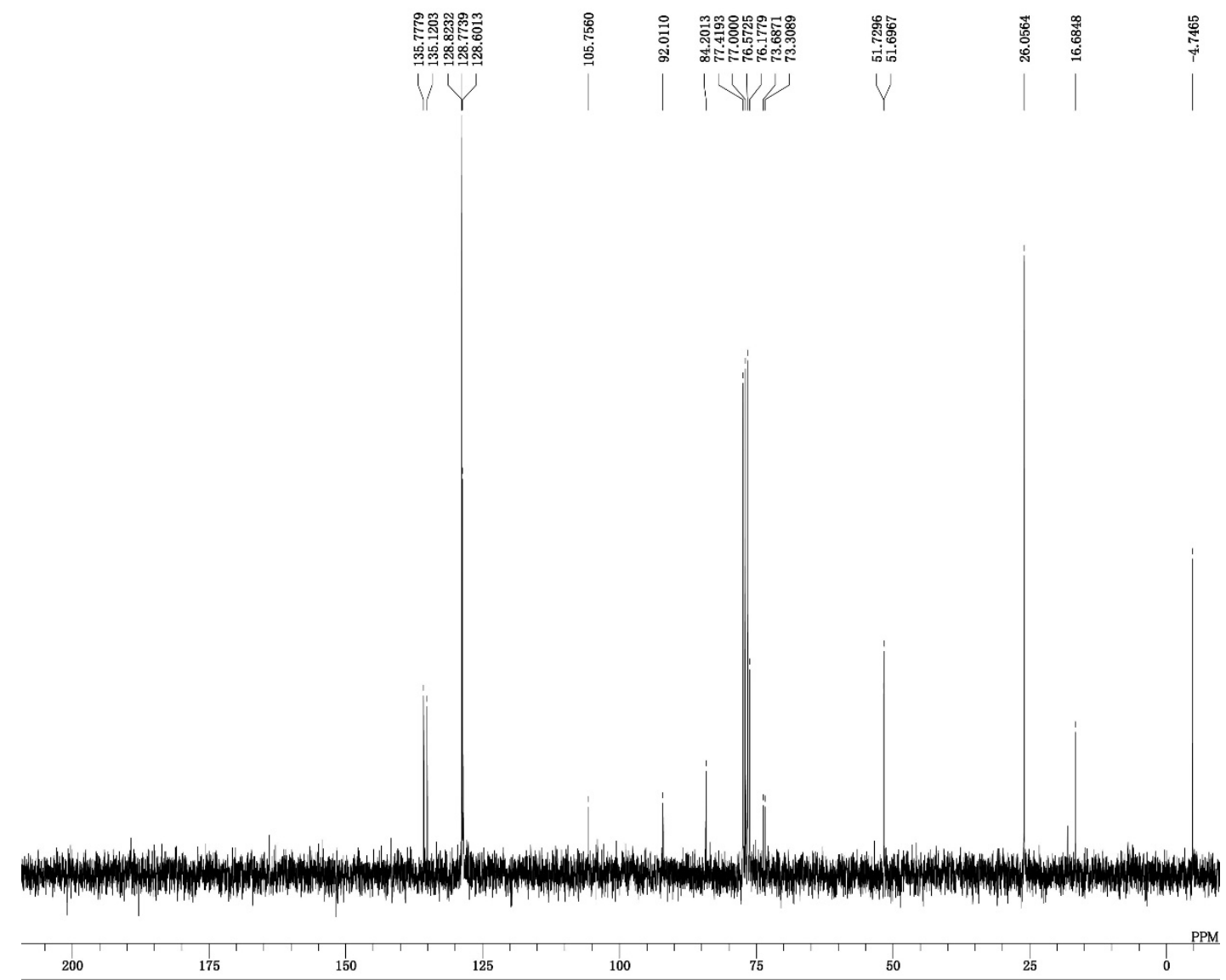




\section{WILEY-VCH}

${ }^{1} \mathrm{H}$ NMR spectrum of $\mathbf{6}$

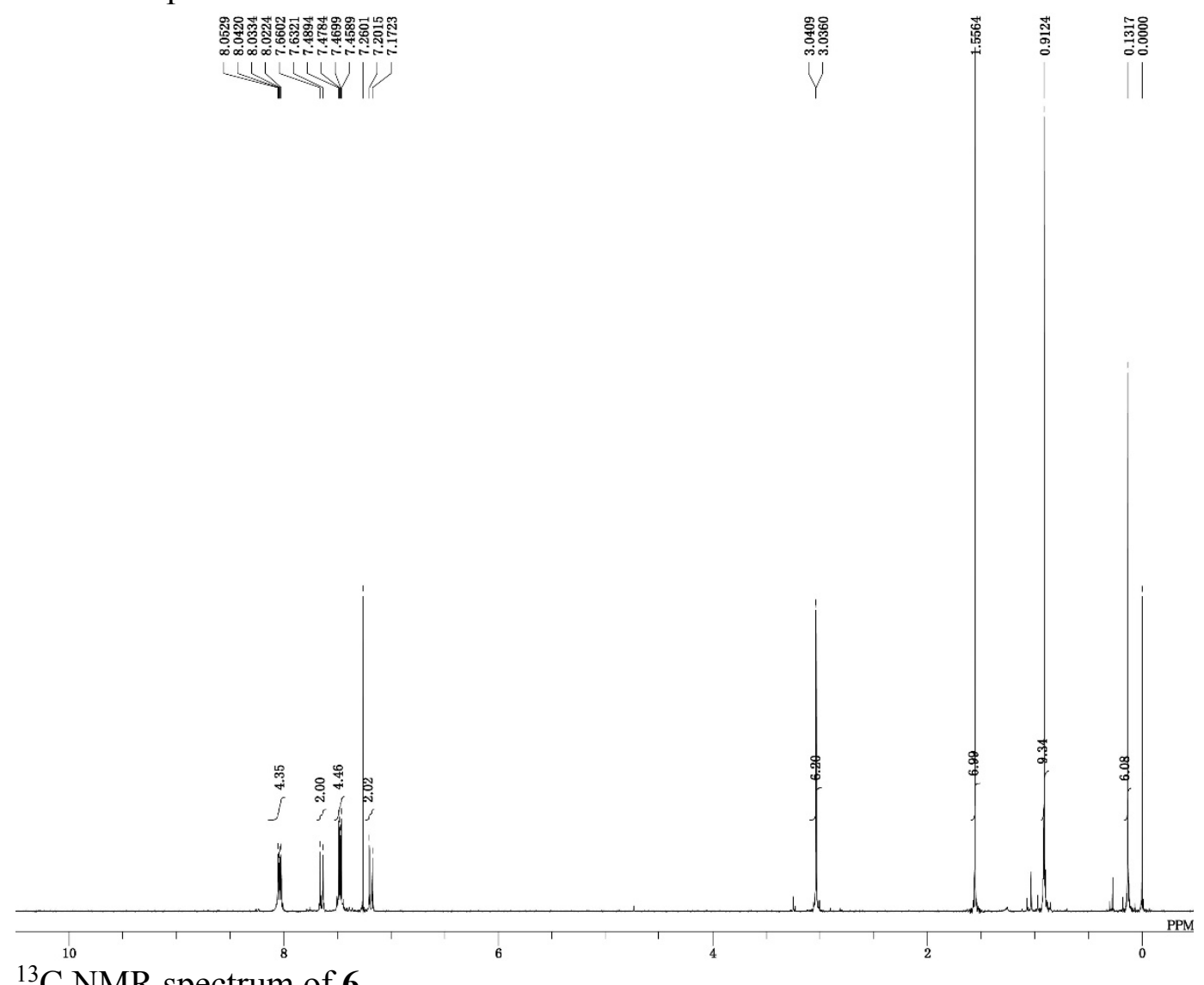

${ }^{13} \mathrm{C}$ NMR spectrum of 6

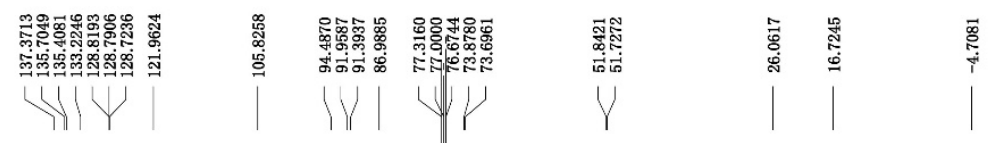
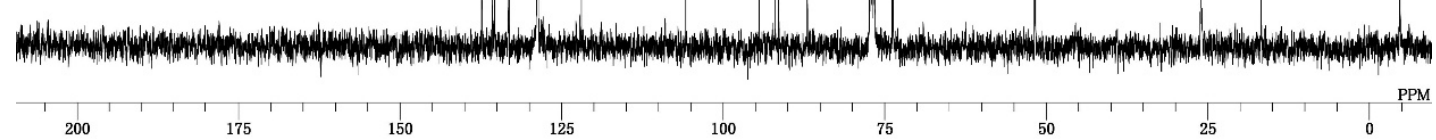

175

150

$\underset{125}{\mid+1}$

100 PPM 
WILEY-VCH

${ }^{1} \mathrm{H}$ NMR spectrum of 7

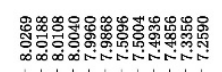

4y
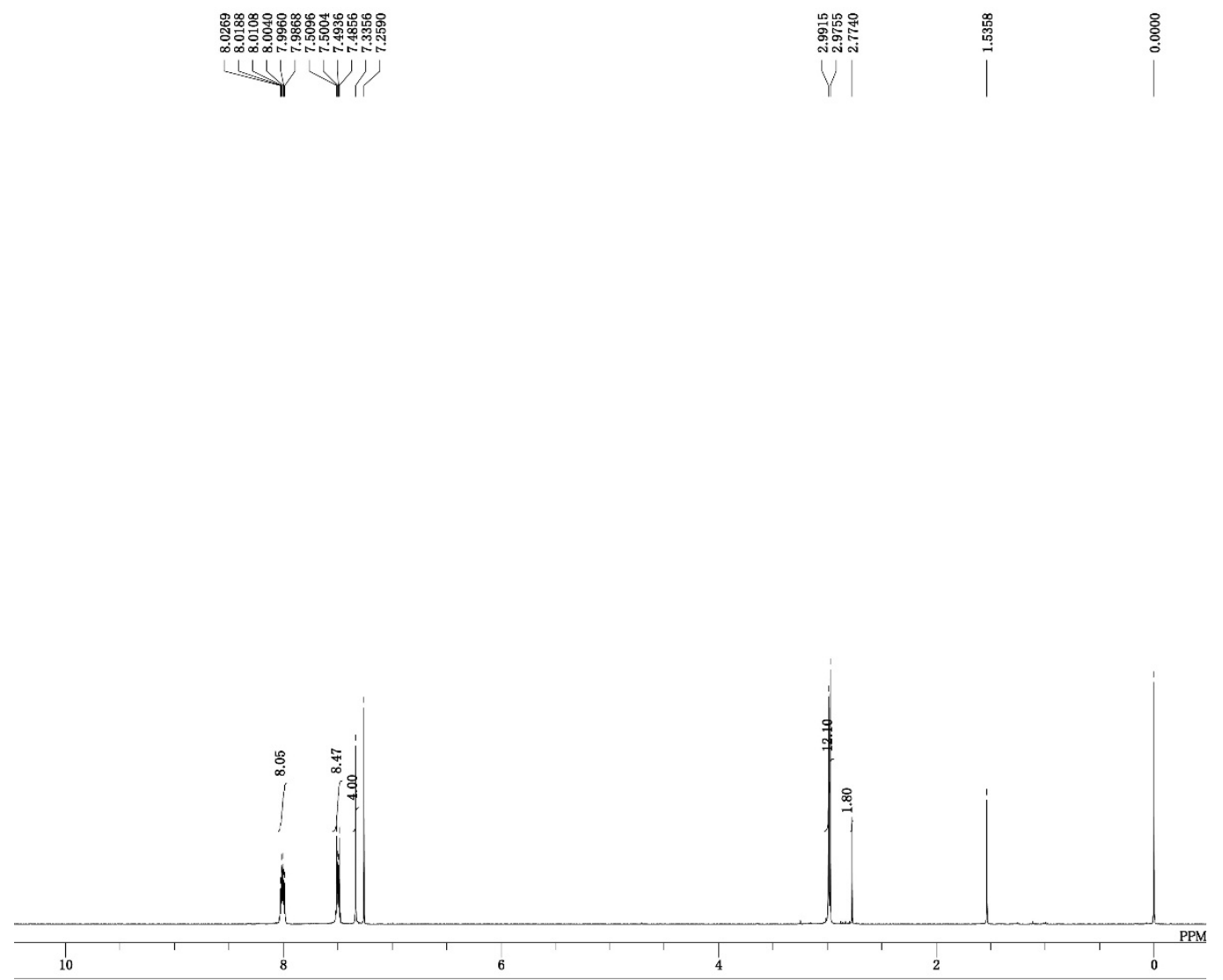

${ }^{13} \mathrm{C}$ NMR spectrum of 7

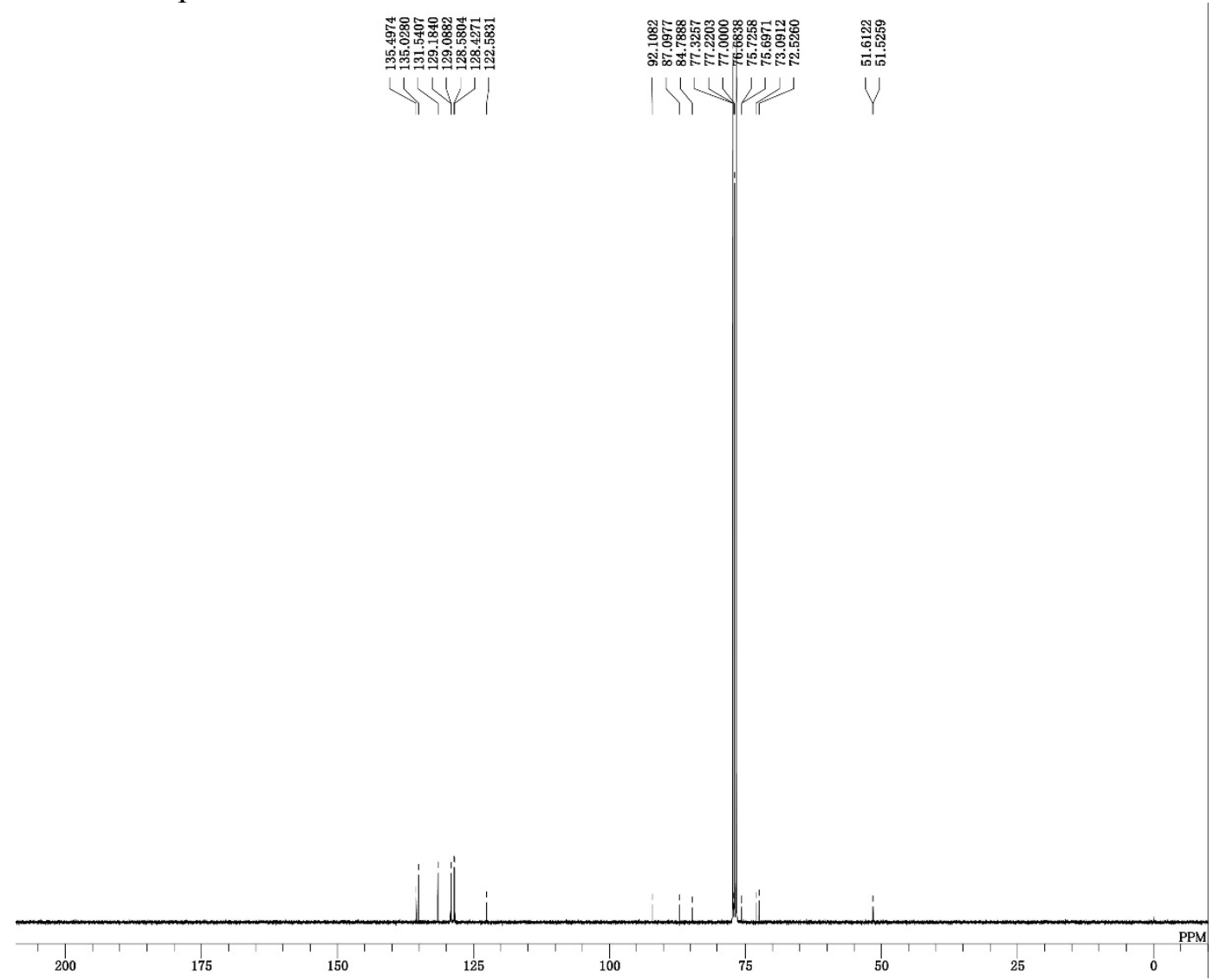




\section{WILEY-VCH}

${ }^{1} \mathrm{H}$ NMR spectrum of 8

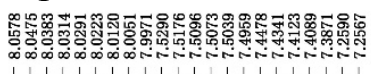

L

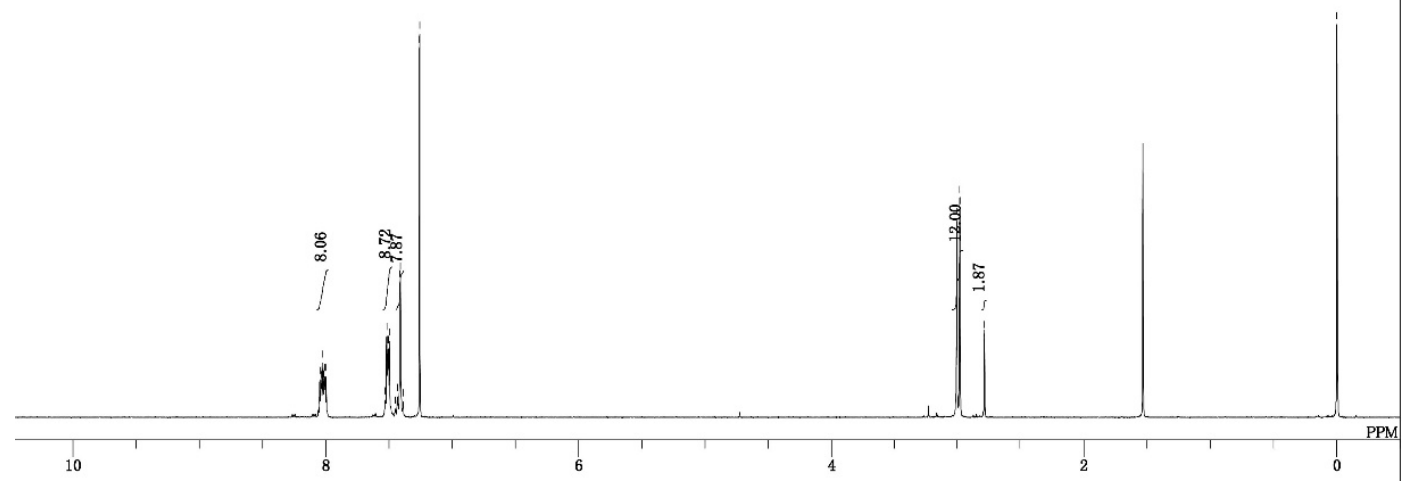

${ }^{13} \mathrm{C}$ NMR spectrum of 8

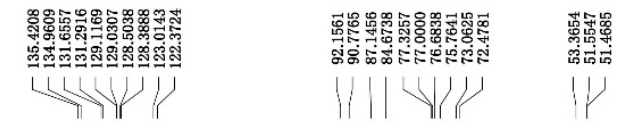




\section{WILEY-VCH}

${ }^{1} \mathrm{H}$ NMR spectrum of 9
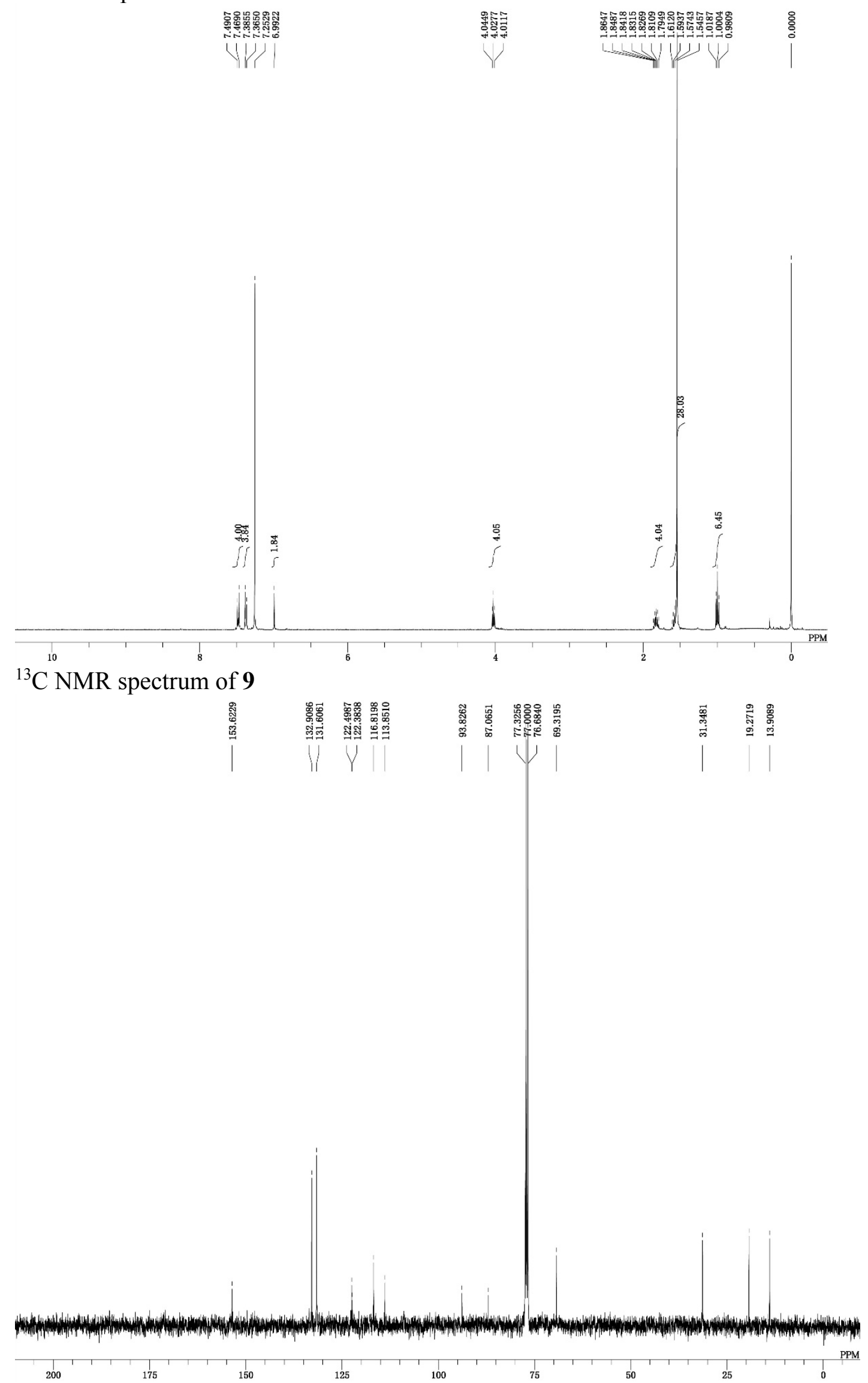


\section{WILEY-VCH}

\section{${ }^{1} \mathrm{H}$ NMR spectrum of $\mathbf{1 0}$}

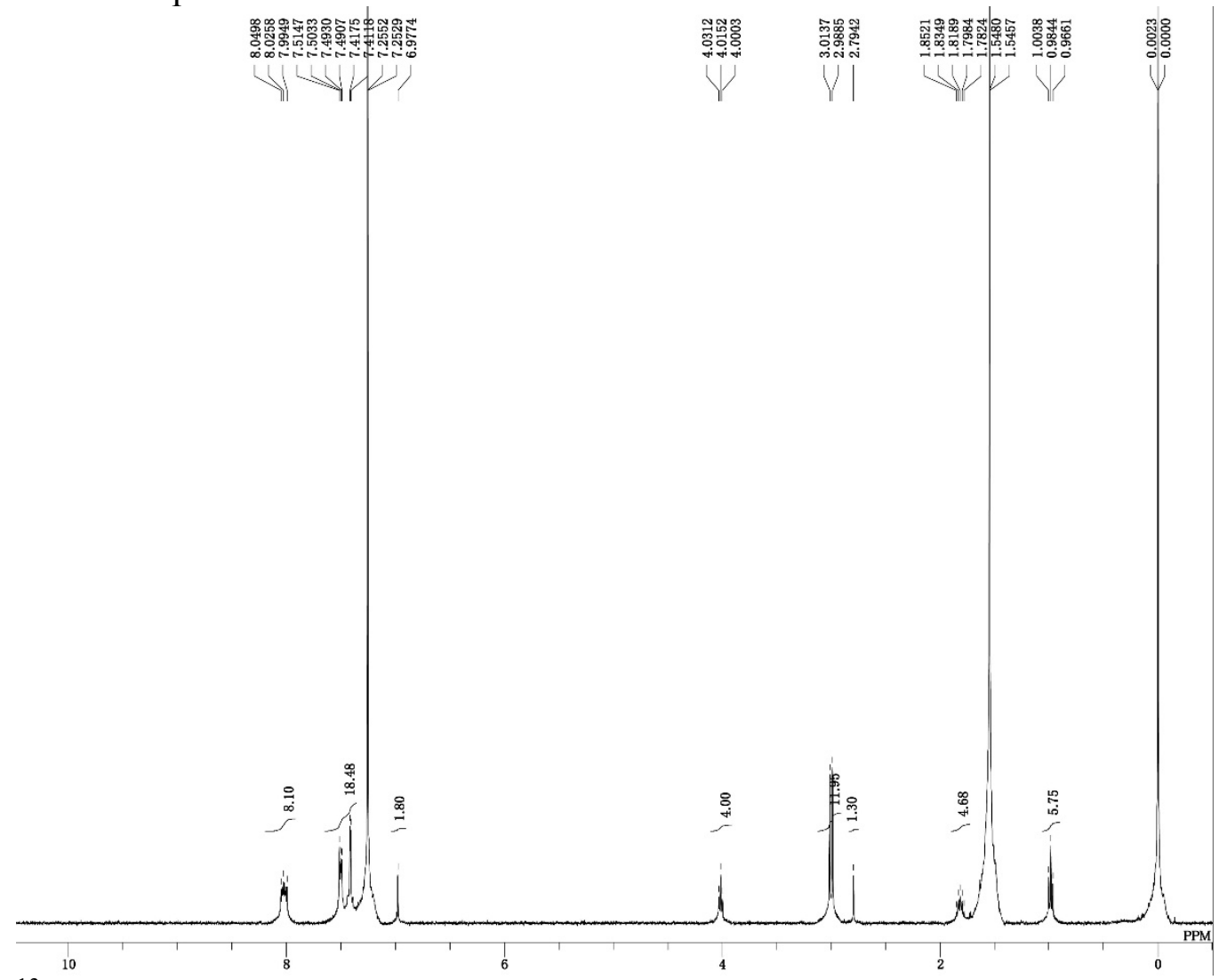

${ }^{13} \mathrm{C}$ NMR spectrum of $\mathbf{1 0}$

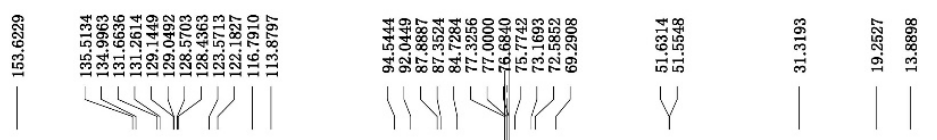

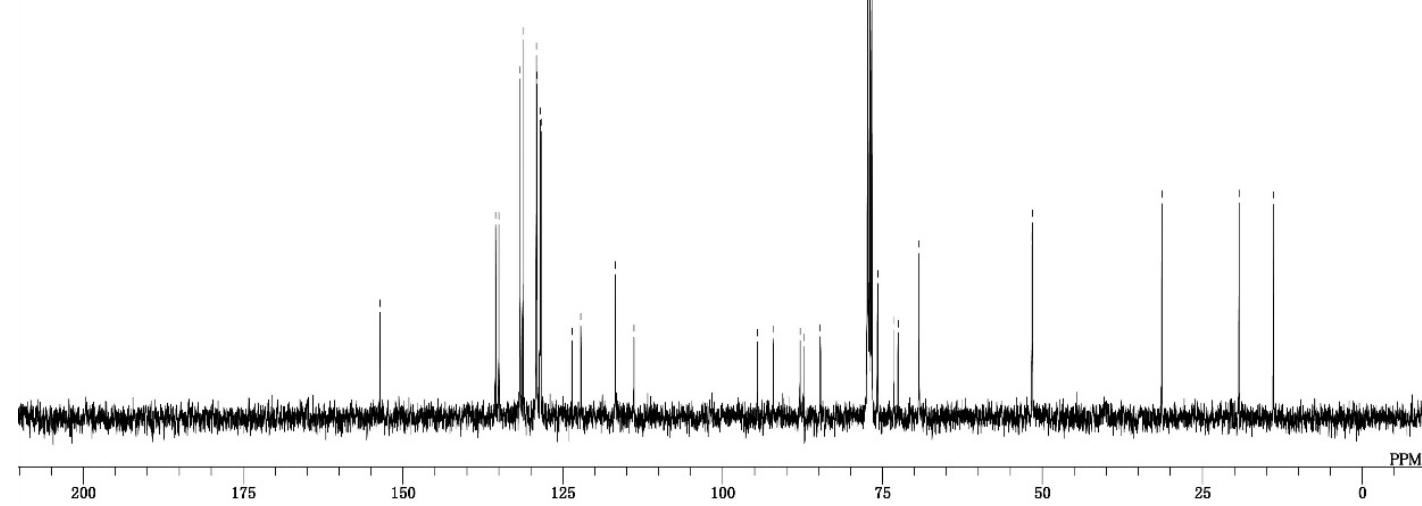




\section{WILEY-VCH}

${ }^{1} \mathrm{H}$ NMR spectrum of $\mathbf{1 1}$

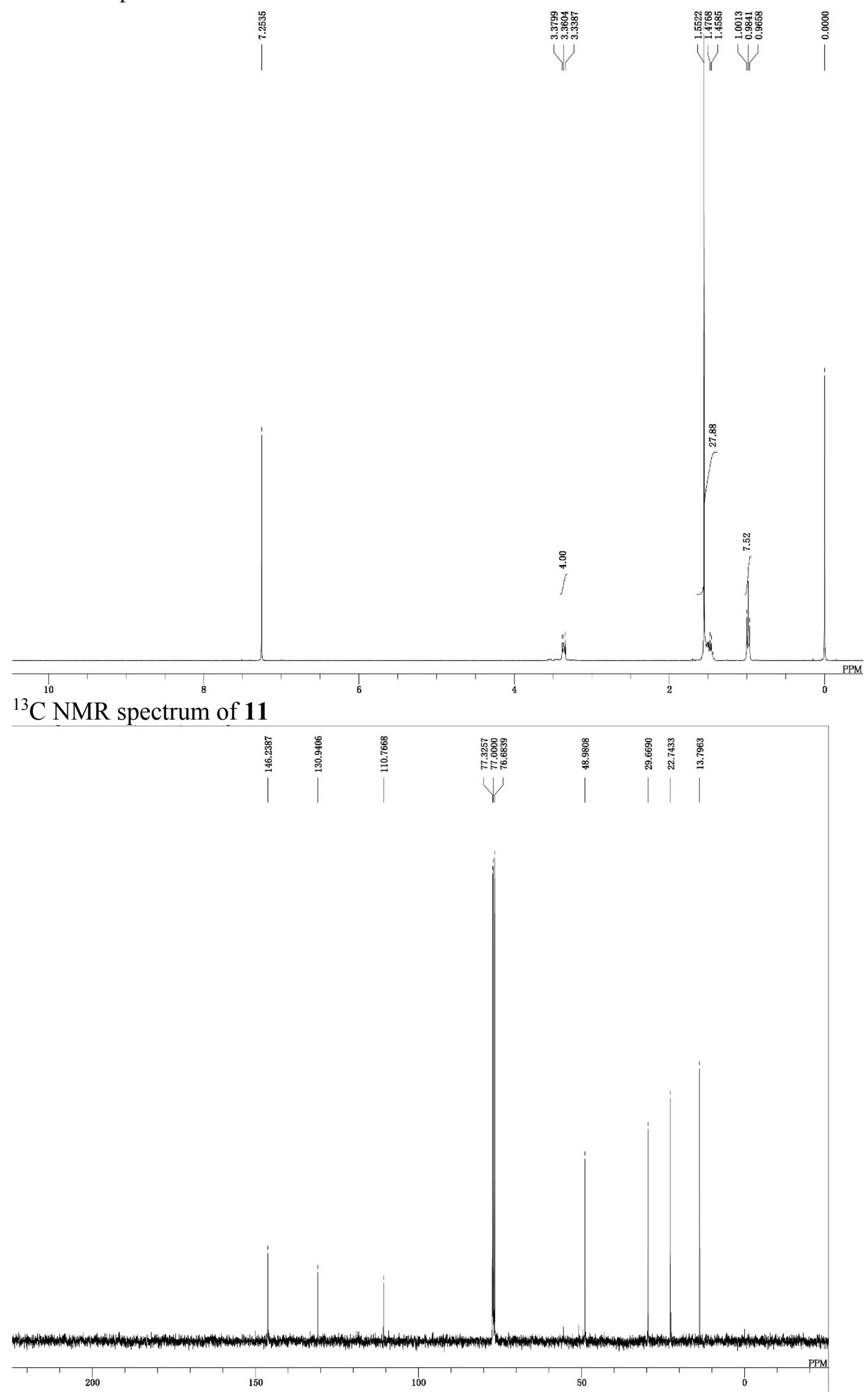




\section{WILEY-VCH}

${ }^{1} \mathrm{H}$ NMR spectrum of $\mathbf{1 2}$
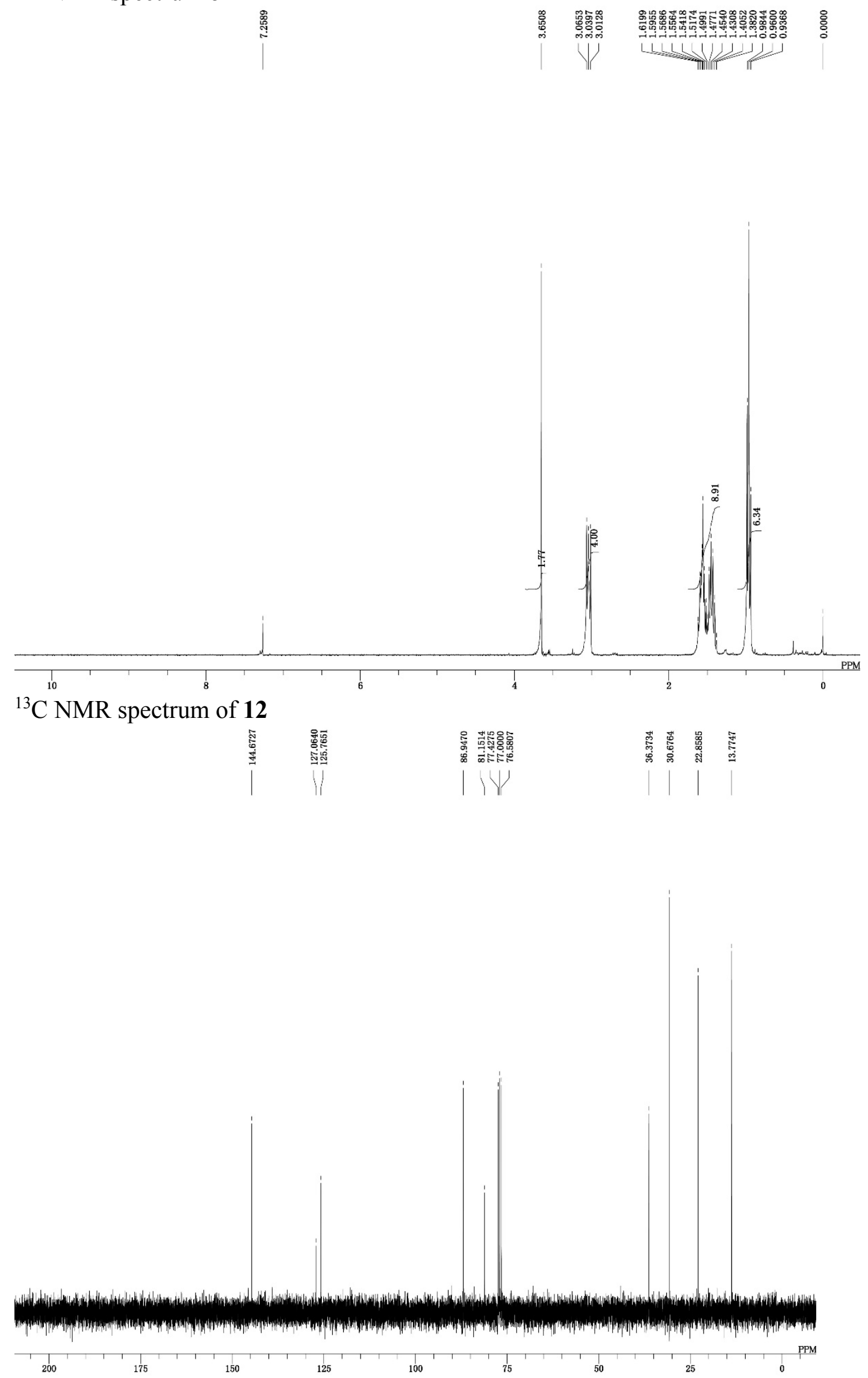


\section{WILEY-VCH}

${ }^{1} \mathrm{H}$ NMR spectrum of $\mathbf{1 3}$

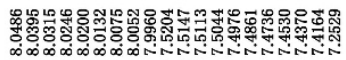

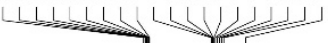

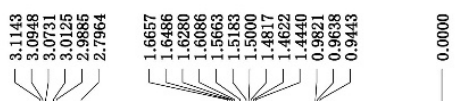

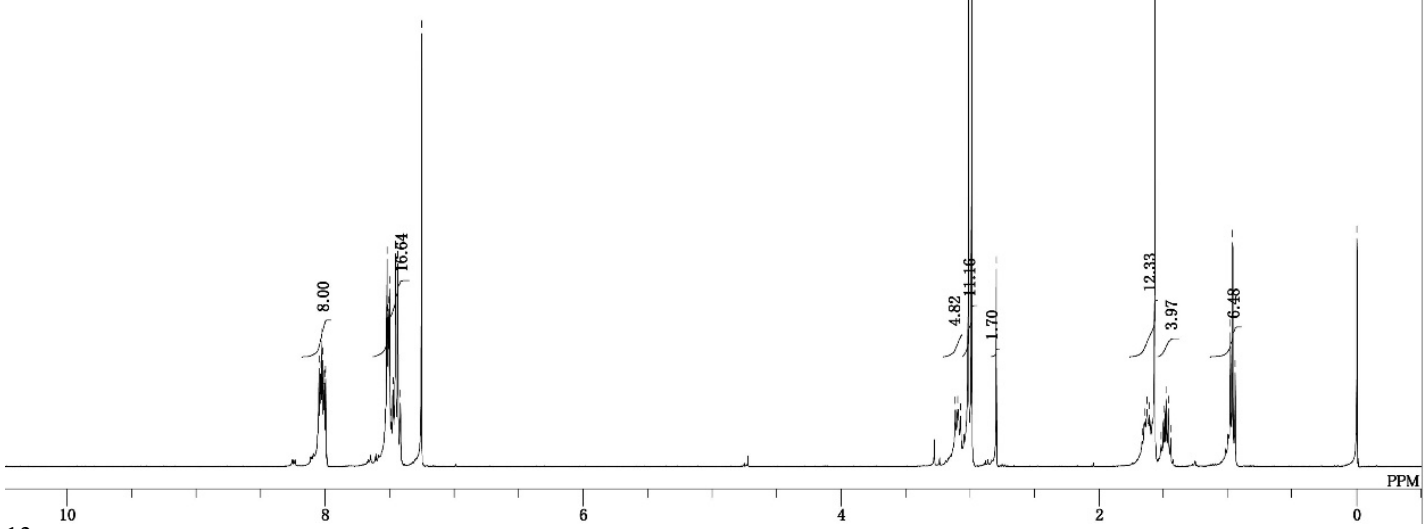

${ }^{13} \mathrm{C}$ NMR spectrum of $\mathbf{1 3}$

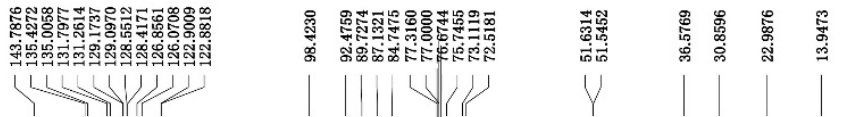

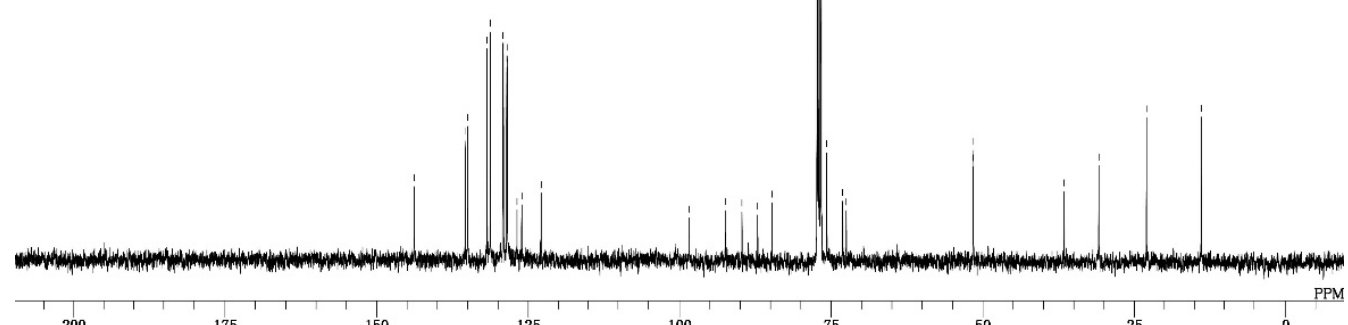


WILEY-VCH

${ }^{1} \mathrm{H}$ NMR spectrum of $\mathbf{1 4 a}$

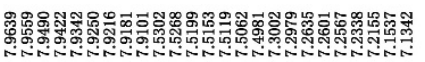

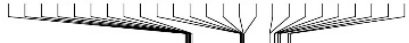

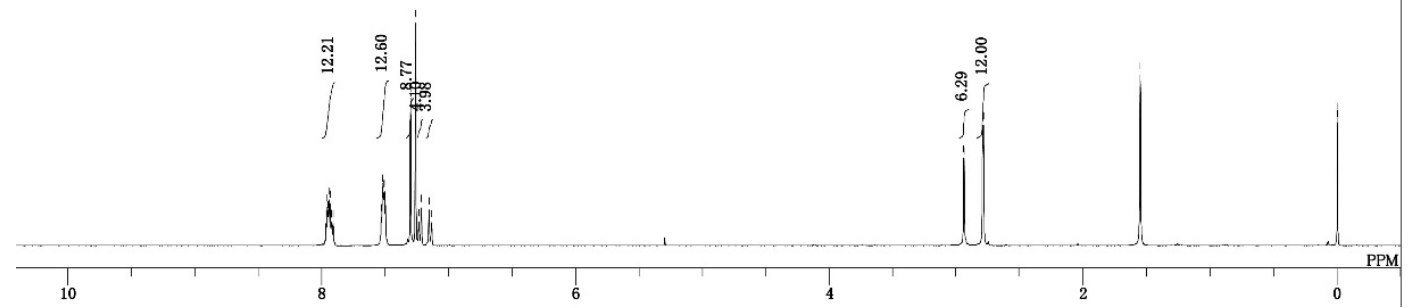

${ }^{13} \mathrm{C}$ NMR spectrum of $\mathbf{1 4 a}$

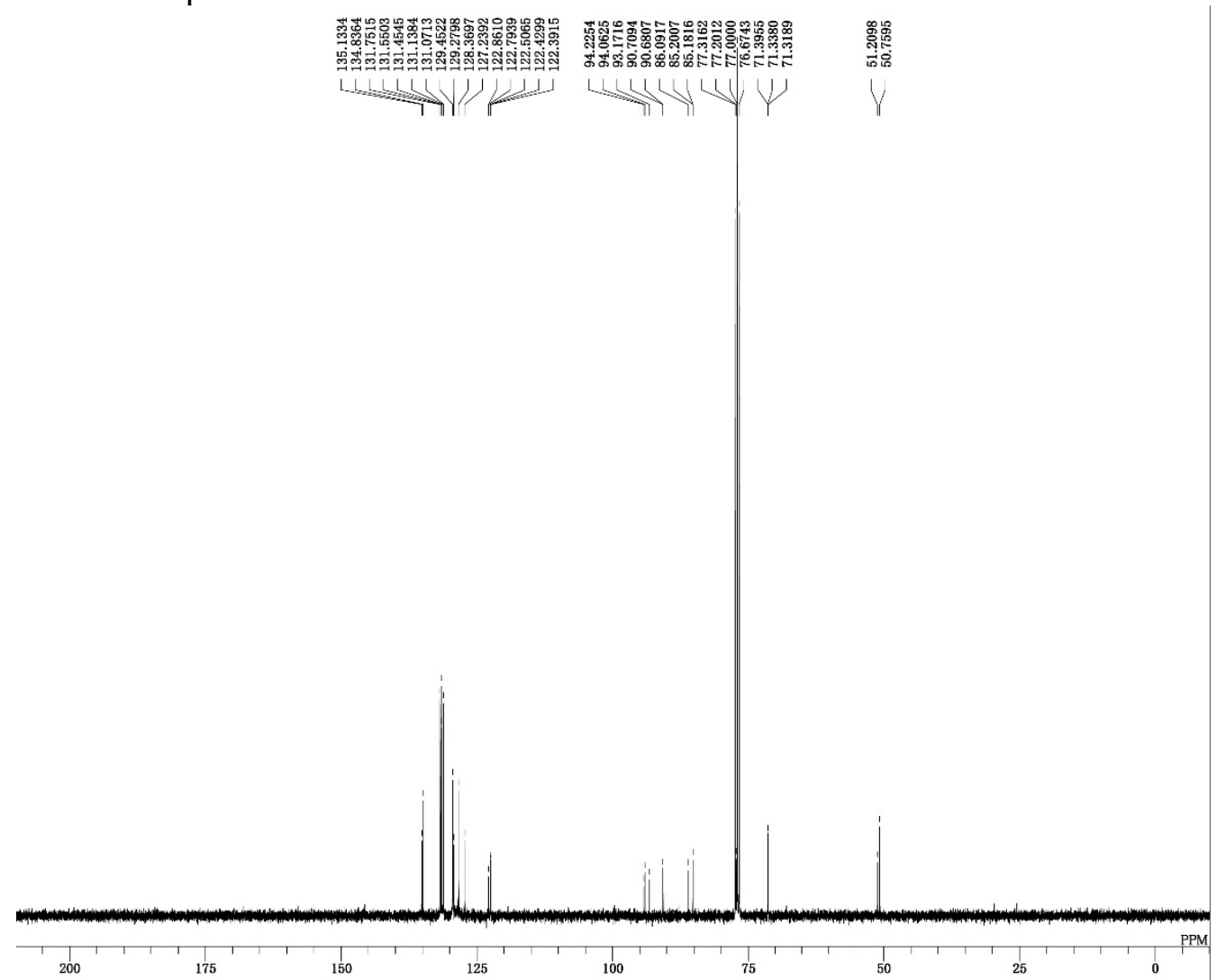




\section{WILEY-VCH}

${ }^{1} \mathrm{H}$ NMR spectrum of $\mathbf{1 4 b}$

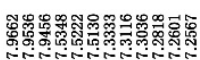

wisw

㖊

$\mid$

:

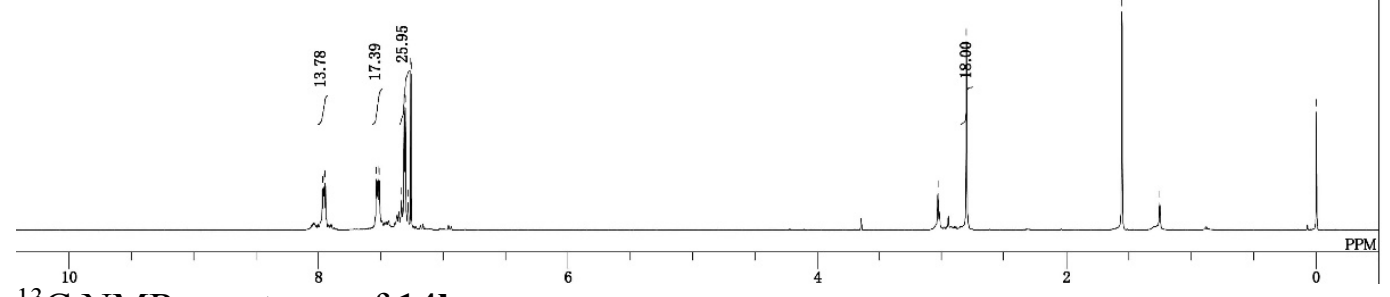

${ }^{13} \mathrm{C}$ NMR spectrum of $\mathbf{1 4 b}$

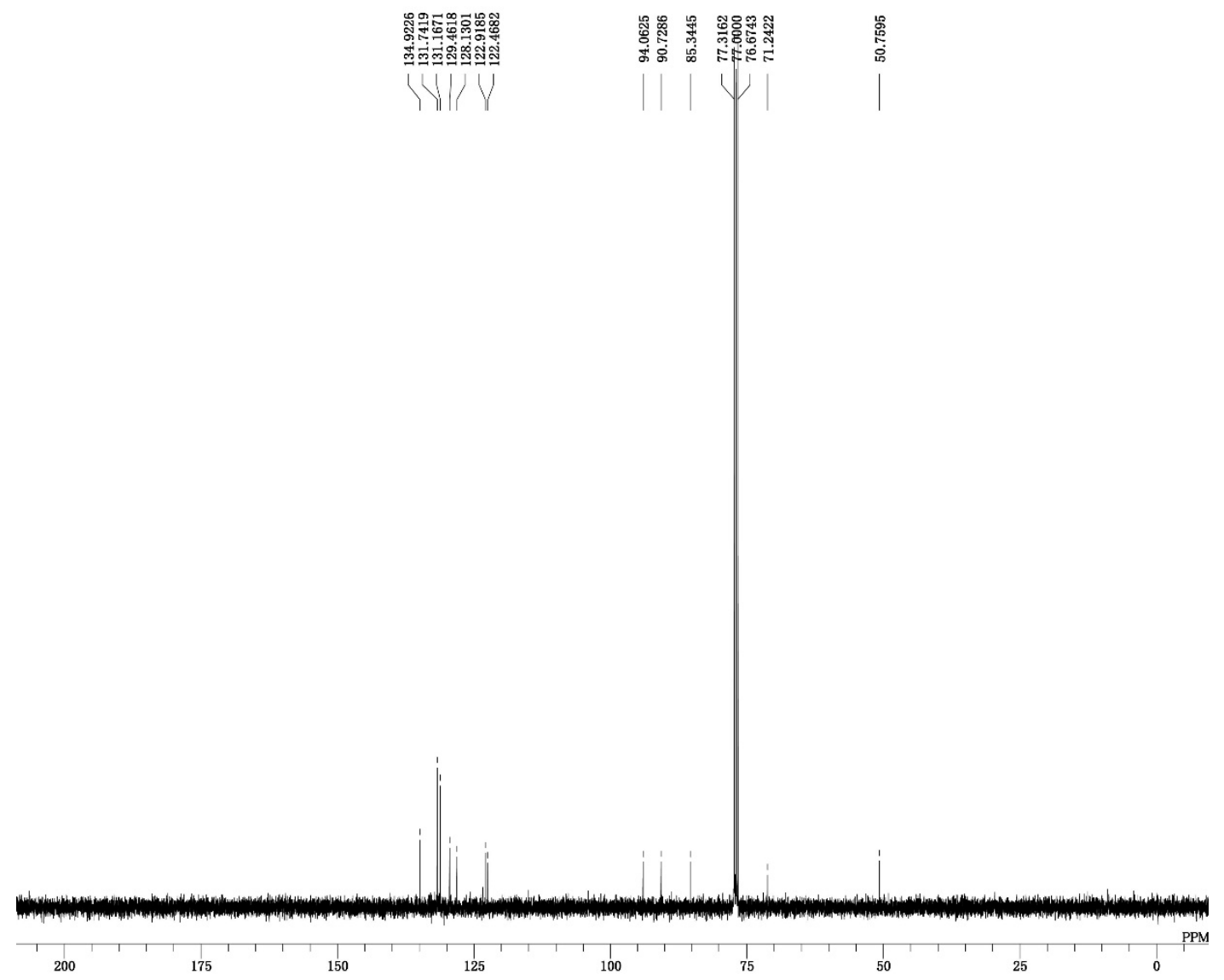




\section{WILEY-VCH}

${ }^{1} \mathrm{H}$ NMR spectrum of $\mathbf{1 4 c}$

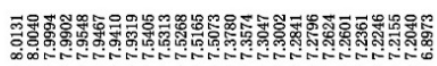

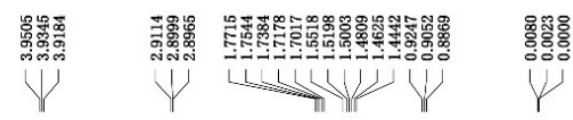
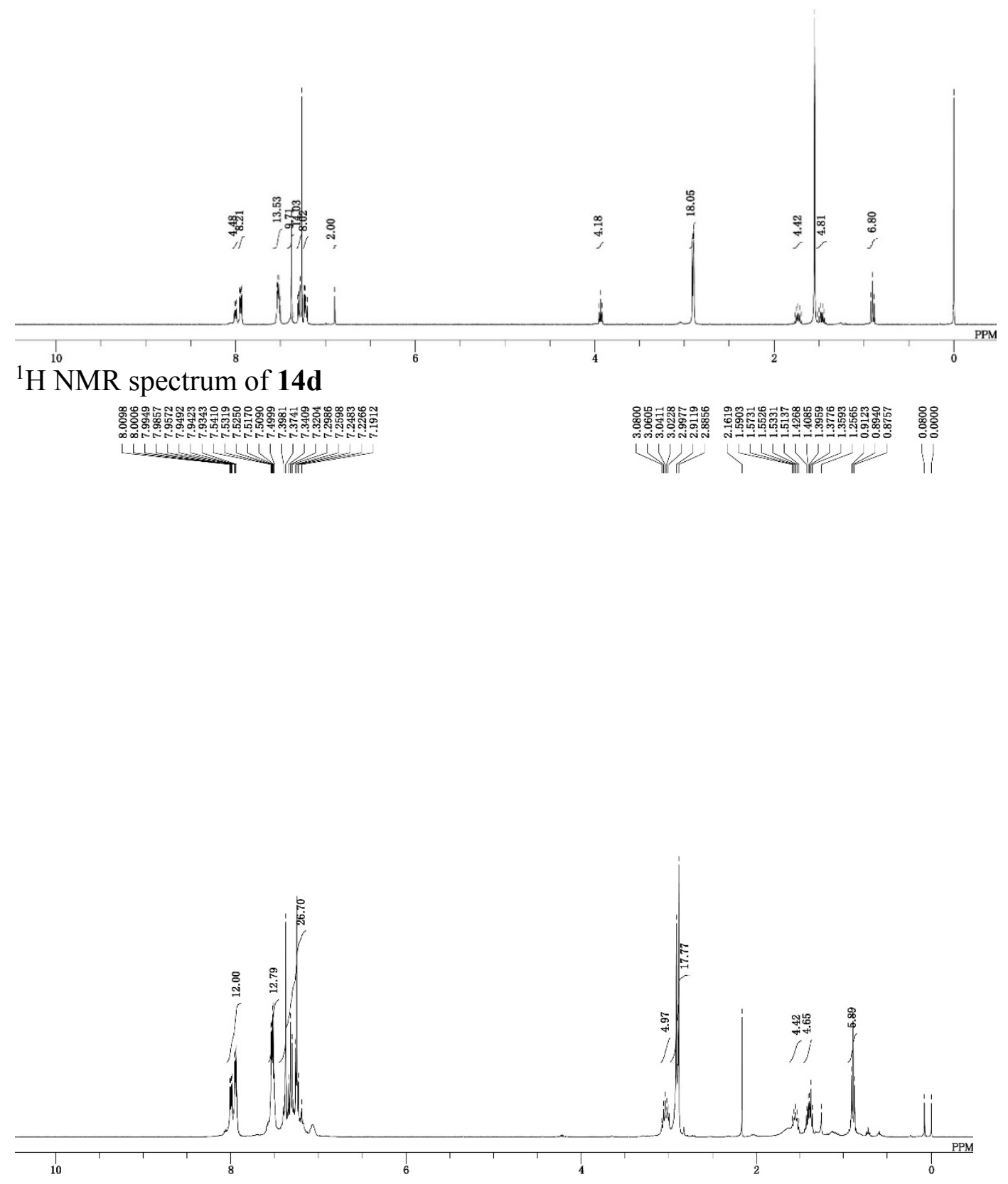